\title{
A comparison of methods for theoretical photochemistry: Applications, successes and challenges
}

\section{Nicholas S. Hill, Michelle L. Coote*}

ARC Centre of Excellence for Electromaterials Science, Research School of Chemistry, Australian National University, Canberra, ACT, Australia

*Corresponding author: e-mail address: michelle.coote@anu.edu.au

\section{Contents}

1. Introduction

2. Photochemical phenomena

2.1 Excited state potential energy surfaces (PESs) 205

2.2 Simulation of spectra

2.3 Other important phenomena

3. Time-dependent Hartree-Fock (TD-HF) 208

3.1 Nonlinear optical properties 208

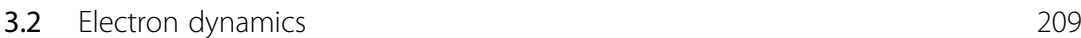

3.3 Supplementing TD-HF with TD-DFT 210

$\begin{array}{lll}3.4 & \text { Assessment } & 211\end{array}$

4. Configuration interaction 211

$\begin{array}{lll}4.1 & \text { Absorption and emission spectra } & 212\end{array}$

$\begin{array}{lll}4.2 & \text { Assessment } & 214\end{array}$

5. Multireference studies 215

$\begin{array}{lll}5.1 & \text { Mechanistic studies } & 216\end{array}$

$\begin{array}{lll}5.2 & \text { Restricted active spaces (RAS) } & 221\end{array}$

$\begin{array}{ll}\text { 5.3 Quantum Monte-Carlo (QMC) } & 223\end{array}$

$\begin{array}{lll}5.4 & \text { Benchmarking studies } & 224\end{array}$

$\begin{array}{lll}5.5 & \text { Assessment } & 227\end{array}$

6. Time-dependent density functional theory 228

6.1 Methodological developments 228

6.2 Dye chemistry 232

6.3 Excited state intramolecular proton transfer 238

$\begin{array}{ll}6.4 & \text { Photoinitiation } \\ 6.5 & 241\end{array}$

$\begin{array}{ll}\text { 6.5 Other photochemical reactions } & 243\end{array}$

6.6 UV-Vis spectra and excitation energies 245

6.7 Thermally activated delayed fluorescence (TADF) 249 
6.8 Fluorescence and quenching $\quad 252$

6.9 Other properties 255

$\begin{array}{ll}6.10 & \text { Assessment } \\ 257\end{array}$

7. Solvent and environmental effects 258

$\begin{array}{lll}7.1 & \text { Continuum versus explicit solvent } 258\end{array}$

$\begin{array}{ll}7.2 & \text { Equilibrium versus nonequilibrium solvation } \\ 7.3 & 262\end{array}$

7.3 Other environmental effects 263

8. Conclusion 264

Conflicts of interest $\quad 265$

Acknowledgments $\quad 265$

References $\quad 265$

$\begin{array}{ll}\text { Further reading } & 284\end{array}$

\section{Abstract}

Herein we highlight recent studies and active areas of interest in the ongoing challenge to model photochemical processes in a wide variety of molecules. We also discuss recent significant methodological improvements and developments that may aid future investigations. Studies using the wide range of techniques available in modern electronic structure software packages have been included, with their successes and shortcomings forming part of the discussion. This study should therefore aid in the design of future computational studies.

\section{Introduction}

Photochemical processes cover a wide-range of physical phenomena that molecules can undergo upon the absorption of radiation, and to this day remain a challenge to model using computational methods. While ground state chemistry is dominated by the relative energies of reactants, products, and the resulting possible transition states, and can be subjected to traditional thermodynamic and kinetic arguments, often in photochemistry these central tenets no longer hold. The interplay of competing processes, generally described as radiative and nonradiative, can result in observed behavior arising due to degeneracies in electronic states, symmetry arguments, the nature of the excited state, often while a molecule is populating a nonequilibrium geometry.

The difficulty in modeling the excited states of molecules initially arises from the failings of the traditional self-consistent field (SCF) approach to variationally solving the time-independent Schrodinger equation. Simply taking, for example, a ground state Hartree-Fock (HF) reference wavefunction and 
promoting an electron into a virtual orbital to form a new, excited state Slater determinant may make physical sense in the description of the state of interest, however, a standard SCF procedure will then simply drive the determinant back to its ground state form, leaving the excited state inaccessible. This variational collapse, while avoidable in some rare, favorable cases, is ubiquitous.

A wide range of theories have therefore been developed that guard against such issues, including theories based on HF wavefunctions, postHF wavefunctions, e.g., coupled-cluster (CC), Møller-Plesset (MPn) perturbation theory, multireference wavefunctions, and Density Functional Theory (DFT). ${ }^{1}$ Dynamic simulations can also be performed using a variety of theories. Each method has its own associated shortcomings and scaling issues, and suitability to modelling different types of photochemical phenomena. This review will therefore aim to determine which methods are favored in recent literature, to assess if progress toward addressing shortcomings has been made, and to highlight continuing challenges. This review builds on previous significant reviews focused on photochemistry by Santoro, ${ }^{2,3}$ Laurent, ${ }^{4}$ Garavelli, ${ }^{5}$ and Serrano-Andres ${ }^{6}$ by focusing on recent studies, namely, those published within the last 5 years. Moreover, our focus is on comparing a range of different theoretical methods and where they were applied, rather than on applications of a single method or advances made within the formalism of the method itself.

\section{Photochemical phenomena}

There are many photochemical effects that can be modeled using the wide-range of computational methods available (Fig. 1); the most important types of phenomena are outlined below.

\subsection{Excited state potential energy surfaces (PESs)}

Invariably, excitation energies along reaction coordinates are essential for gaining mechanistic insight into photochemical reactions. Accurate energies can, for example, show how transition state energies for a reaction can decrease upon excitation, or how a reaction coordinate can become dissociative upon excitation, leading to homolysis. They are also necessary for simulation of radiative decay processes, e.g., emission energies, as these are dependent on the relaxed geometry of the molecule of interest. PESs are also important as observed photochemical behavior is often not defined by a single excited state, instead, population of a certain state can take place followed by crossing to a different state on which the reaction takes place, 


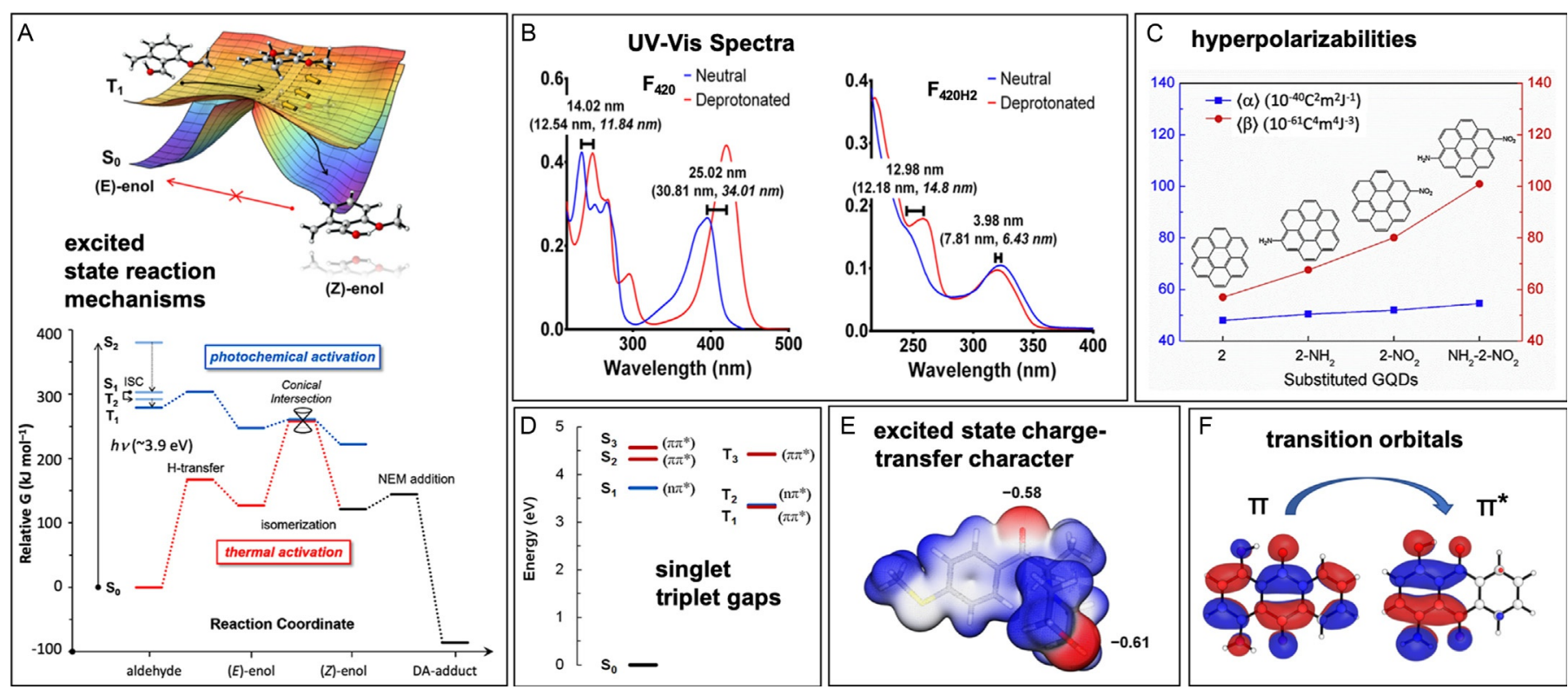

Fig. 1 Typical examples of the types of photochemical phenomena that can be modelled using theoretical chemistry: (A) the excited reaction mechanism for keto-enol tautomerization of an o-methyl benzaldehyde derivative (methyl 4-((2-formyl-3-methylphenoxy)methyl)benzoate) that is subsequently trapped in a Diels-Alder reaction ${ }^{7}$; experimental and simulated UV-Vis spectra for the deazaflavin dependent nitroreductase enzyme bound cofactor $\mathrm{F}_{420}$ and its reduced form $\mathrm{F}_{42 \mathrm{OH} 2}$ in their respective neutral and deprotonated forms ${ }^{8}$; (C) electronic polarizability and second hyperpolarizability of graphene quantum dots as a function of substituent ${ }^{9}$; the (D) vertical singlet-triplet excitation energies and (E) electrostatic potential surface of the $\mathrm{T}_{1} \pi \pi^{*}$ excited state of 2-Methyl-4'-(methylthio)-2morpholinopropiophenone ${ }^{10} ;(F)$ the orbitals involved in the $S_{1} \pi \pi^{*}$ transition of 1-amino-4-hydroxyanthraquinone. ${ }^{11}$ Figures adapted with permission from the original references. 
known as nonradiative processes. ${ }^{12}$ The proper capture of the nature of electronic (near) degeneracies, either at conical intersections or avoided crossings, can be required when quantitative insight into the photochemical mechanism(s) is sought. As shown below, not all methods can produce such accuracy; however, qualitative insight may only be necessary, and approximate PES can often be obtained from cheaper methods.

\subsection{Simulation of spectra}

Many implemented excited state codes, where $n$ excited states are obtained variationally, can compute oscillator strengths and transition dipole moments between the $\mathrm{S}_{0}$ and the $n$th excited state; this information can be coupled with the corresponding excitation energy to simulate different types of spectra. Simulation of spectra can provide invaluable insight into the initial photochemical processes taking place upon excitation; UV-vis spectra are often experimentally available and the similarity between the computed and experimental spectra can suggest the excitation features are being correctly modelled. Modern implementations also include the orbitals involved in the transition, from which it is possible to determine the nature of the excited state; for example, is the lowest energy transition to a bright $\pi \pi^{*}$ state, as can be observed in both computed and experimental spectra, or is it a dark $n \pi^{*}$ state, which won't be visible but could be photochemically relevant? Emission spectra can also be computed using the relaxed excited state geometry, from which Stokes' shifts can be estimated. Often, absolute accuracy of the computed spectra is not necessary; instead, the capture of trends may be more important. For example, one may be attempting to tune a certain property of a molecule (e.g., HOMO-LUMO gap), for which a range of substituents may be tested. Proper capture of the substituent effects (e.g., red-shift vs blue-shift) on the property therefore provides insight for targeted design.

\subsection{Other important phenomena}

Excited state PESs and simulated spectra are two popular uses for excited state methods; other phenomena that can be modeled include the following.

Singlet-triplet energy gaps $(\Delta E S T)$. Accurate values of $\Delta \mathrm{E}_{\mathrm{ST}}$ can provide insight into the likelihood of singlet-triplet crossing upon excitation.

Charge-transfer (CT) vs local excitation (LE). An excited state with CT character involves a significant shift in electron density to different region of a molecule. These states can have profound effects on a molecule's optical 
properties, yet can be challenging to model due to self-interaction error ${ }^{13}$ (in DFT), and significant orbital relaxation being required.

Nonlinear optical effects. Not all photochemistry necessarily arises from the absorption/emission of a single photon. It is therefore sometimes necessary to compute two-photon cross-sections, for when two-photon absorption is observed. It is also sometimes necessary to compute high-order hyperpolarizabilities, which can give rise to, for example, high-order harmonic generation effects.

\section{Time-dependent Hartree-Fock (TD-HF)}

HF theory is the theoretical cornerstone for all ab initio quantum chemistry theories, and therefore provides a natural starting point for extension to excited state. The main excited state HF-based methods are configuration interaction with single and double substitutions (CIS and CISD, respectively), and time-dependent HF (TD-HF). However, HF theory infamously neglects dynamic electron correlation. In certain situations, for example when modeling isodesmic reactions, HF can provide quantitative accuracy due to favorable error cancellation; however, in excited states where orbital occupancies are different to the ground state, and where the excited state electron density will be necessarily more diffuse, there is no reasonable expectation for the electron correlation energy of the two states to cancel. CIS(D) and TD-HF do, however, benefit from favorable scaling with respect to system size, so can be employed where high-level, high-accuracy ab initio calculations are simply not feasible.

\subsection{Nonlinear optical properties}

Recent studies ${ }^{14-17}$ employing TD-HF have focused on investigating nonlinear optical (NLO) properties, predominantly due to the implementation of the relevant calculations required to compute these properties. Karakas et al. ${ }^{16}$ investigated the two-photon absorption (TPA) cross-sections, electric dipole moment, and the second-order hyperpolarizability of tetrathiafulvalene derivatives, with and without $\operatorname{Pt}(\mathrm{II})$ and $\operatorname{Pd}(\mathrm{II})$. The authors claim good agreement with previous experimental data, despite the theoretical values for isotropic second hyperpolarizabilities being overestimated, compared to experimental data, by between 1 and 2 orders of magnitude. Guezguez et al. ${ }^{17}$ performed a similar study on Au(III) dithiolene complexes, in which electric dipole moments, dispersion-free and frequency-dependent dipole polarizability, and second hyperpolarizability 
tensor components were calculated. The calculations show much closer agreement with experimental values of third order susceptibility $\left(\mathrm{X}^{3}\right)$ than from similar calculations presented in Karakas ${ }^{14,16}$ and Zawadzka ${ }^{15}$ (2015) employ similar calculations but this time to styrylquinolinium dyes and metallophthalocyanine chlorides, respectively. In these four studies, TD-HF is successful as the calculation of second- and third-order nonlinear properties is relatively trivial, and the inaccuracy of TD-HF is largely irrelevant; instead, the studies utilize the results for qualitative trends, rather than for mechanistic arguments.

In a similar vein, $\mathrm{Wu}$ et al. ${ }^{18}$ used TD-HF to calculate second-order hyperpolarizabilities on a variety of increasingly substituted triarylamine chromophores, finding that addition of several methoxy groups resulted in a large increase in hyperpolarizability, compared to the unsubstituted molecule. This is likely due to the increased electron density in the $\pi$-system of the molecule, and this conclusion is supported by that of Alparone, ${ }^{19}$ who showed that increasing fluoro-substitution of benzene, and hence decreasing electron density in the $\pi$-framework, results in a decrease in calculated second-order hyperpolarizability. Alparone used MP2 to calculate the second-order hyperpolarizabilities as well as TD-HF, and both sets of results are in good agreement. The TD-HF results are systematically underestimated for all 13 molecules investigated compared to the MP2 results, however, trends are identical.

NLO studies have also been performed using TD-HF on a dithienylethene-indolinooxazolidine-benzazolooxazolidine system, ${ }^{20}$ on merocyanine dyes, ${ }^{21}$ on an explicitly benzene-solvated nitrobenzene system, ${ }^{22}$ on triarylamine (TAA) chromophores, ${ }^{18}$ on fluorobenzenes, ${ }^{19}$ on 2-aminobenzimidole complexes, ${ }^{23}$ and on 3-(4-methoxyphenyl)-1(4-nitrophenyl)prop-2-en-1-one (MNC).$^{24}$ All of these studies involve relatively large molecules ( $>20$ heavy atoms) and therefore benefit from the relative inexpensive HF calculations.

\subsection{Electron dynamics}

Studies by Lötstedt et al. ${ }^{25}$ and $\mathrm{Hu}$ et al. ${ }^{26}$ use TD-HF to study the electron dynamics of acetylene upon exposure to an intense laser field; here using time-dependent in the literal, rather than theoretical, sense. Luzanov ${ }^{27}$ and Nguyen and Parkhill ${ }^{28}$ also apply TD-HF to nonstationary photochemical behavior. Nguyen and Parkhill use real-time TD-HF as a comparison for their newly developed time-dependent open self-consistent field at 
second order (OCSF2) propagation method on 4-((4-(dimethylamino)phenyl)buta-1,3-diyn-1-yl)benzonitrile, demonstrating that their method can cope with nonadiabatic dynamic behavior. Sato and Ishikawa ${ }^{29}$ also applied TD-HF to laser-field ionization dynamics, using a one-dimensional helium atom as the test case, as a full numerical solution to the dynamic ionization process in this system is possible. However, they found that the closed shell TD-HF model is insufficient to model the relatively extreme dynamics produced upon exposure to strong lasers.

\subsection{Supplementing TD-HF with TD-DFT}

Bondu et al. (2015), Bruckner et al. (2015), and Maidur et al. (2017) use a combined TD-HF and DFT approach, with the increase in electron correlation included in DFT benefitting the thermodynamic and equilibrium properties of the molecules of interest. The main focus of the work by Bruckner et al. (2015), however, was to study the excited state reorganization energies. Bondu et al. (2015) also used TD-DFT for comparison with TD-HF results for linear (i.e., one-photon absorption) properties of different isomers of dithienylethene-indolinooxazolidine-benzazolooxazolidine, finding in general that while TD-HF overestimated vertical excitation energies, it was able to provide qualitative trends for each of the four types of molecule investigated, in agreement with TD-DFT results. Bruckner et al. also initially employed TD-DFT, but quote "charge-transfer contamination" in their TD-DFT calculations as the reason for instead using TD-HF. It is unclear if usage of a long-range corrected DFT functional, for example, CAM-B3LYP rather than B3LYP, could have improved TD-DFT results.

Like Bondu et al. (2015), Maidur et al. (2017) also found that their TD-HF absorption spectrum is blue-shifted compared with TD-DFT calculations. There is also significant deviation in the computed oscillator intensities among the DFT functionals used (B3LYP, CAM-B3LYP, and $\omega \mathrm{B} 97 \mathrm{X}-\mathrm{D})$, and also between TD-DFT and TD-HF calculations. The main transitions of interest involve the $\mathrm{HOMO} \rightarrow \mathrm{LUMO}$ and $(\mathrm{HOMO}-1) \rightarrow$ $(\mathrm{LUMO}+1)$ orbitals, which appear to be $\pi \pi^{*}$ and $n \pi^{*}$ transitions, respectively, and it is unclear whether TD-HF and TD-DFT correctly capture this ordering. TD-HF vertical excitation energies and oscillator strengths also show significant deviation from TD-DFT results when applied to 1-[N-(2-pyridyl) aminomethylidene]-2(1H)-napthalenone. ${ }^{30}$ For each of these studies, when necessary, TD-HF was exclusively used to calculate 
the higher order polarizabilities of the molecules of interest; predominantly, these results were used for internal comparisons, rather than being compared to experimental values.

\subsection{Assessment}

It is clear from the relatively small number of studies over the past 5 years, that TD-HF is not a favored technique for providing chemical insight into the behavior of photo-excited molecules. However, the favorable scaling allows its application to large systems, and the wide range of nonlinear properties that can be calculated do allow for the study of less common photochemical phenomena (Table 1). Mechanistic arguments have not generally been drawn from TD-HF results, however, TD-HF has been shown to be able to qualitatively reproduce observed trends and can be used for comparison against higher level calculations.

\section{Configuration interaction}

A more popular HF-based theory is configuration interaction singles, and its variants with and without double excitations (CIS, CISD, CID, CIS(D)), and in recent years, this theory has been applied to a much wider range of molecules than TD-HF. CISD benefits compared to TD-HF due to inclusion of double excitations in its formalism, and can lead to improvements in the description of ground state dissociation processes; however this increase in accuracy comes at increased computational cost. CIS is essentially TD-HF within the Tamm-Dancoff approximation, ${ }^{31}$ generally overestimates excitation energies, and has been found to systematically overestimate charge-transfer states. ${ }^{32}$ However, given the lightweight HF wavefunction

Table 1 TD-HF summary.

Property

Trends

Quantitative

\begin{tabular}{lll}
\hline Nonlinear optical properties & Yes & Rarely $^{\mathrm{a}}$ \\
\hline
\end{tabular}

\begin{tabular}{lll}
\hline Absorption Spectra & No & No \\
\hline Fluorescence Spectra & No & No \\
\hline Photochemical mechanisms & No & No \\
\hline Quantitative photochemical kinetics & No & No \\
\hline
\end{tabular}

${ }^{a}$ Depends on the system and whether it is a first, second, third order hyperpolarizability, with higher order polarizabilities modelled better than lower order ones. 
and lack of post-HF corrections, it remains the fastest excited state method, and can therefore be applied to large systems. Moreover, CIS can also give significantly improved estimations of triplet excitation energies compared to TD-HF. ${ }^{33}$

\subsection{Absorption and emission spectra}

Nithya et al. ${ }^{34}$ utilized a combined CIS and TD-DFT approach to investigate the structural and electronic properties of cyclopentadithiophene derivatives, using the good agreement of TD-DFT calculated vertical excitation energies to simulate absorption and emission spectra, but using optimized excited state geometries as calculated by CIS. Cebrián et al. ${ }^{35}$ employed a similar approach in their study of the photophysical properties of several alkenyl-1H-,1,2,4-triazole compounds. Here, the PBE0 functional was used for ground state geometries and absorption/emission simulations, with the emission calculations being performed on CIS excited state geometries. It is found that utilizing TD-PBE0 excited state geometries resulted in emission errors an order of magnitude larger than those calculated from CIS. Tathe et $a{ }^{36}{ }^{36}$ studied the excited state intramolecular proton transfer of $2-\left(2^{\prime}, 6^{\prime}-\right.$ dihydroxyphenyl)benzoxazole, again using CIS and TD-DFT to calculate absorption and emission spectra. It is found that the TD-DFT results were in much better agreement for both types of spectra than those calculated by CIS, which provided absorption and emission maxima that varied by $\sim 25 \%$. As a result, TD-DFT was favored for modelling the full excited state intramolecular proton transfer.

Laurent et al. ${ }^{37}$ examined the basis set dependence of CIS(D), TD-DFT, second-order polarization propagator approximation (SOPPA), ADC(2), $\mathrm{CC} 2$, and EOM-CCSD, as well as some spin-scaled variants, on the vertical excitation energies of nine different molecules. It was found that spinopposite scaled CIS(D) (SOS-CIS(D)) displayed the largest basis set dependence and generally the largest errors in vertical excitation energies, and ordinary CIS(D) did not fare much better. The study generally shows that, for calculations of vertical excitation energies, CIS(D) is not an accurate method, compared with DFT and higher-level ab initio methods. It also demonstrates that spin-scaling for CIS(D) does not constitute a systematic fix for the shortcomings of CIS(D). CIS was also used as a starting point for the further, higher-level calculations of the excited states of hypobromous $\operatorname{acid}^{38}$; no comment is made however on the accuracy of the CIS calculations. Another benchmarking study was performed by Shinde, ${ }^{39}$ in which CIS, 
EOM-CCSD, random-phase approximation (RPA), and various DFT functionals are applied to the linear absorption properties of boron wheels. EOM-CCSD excitation energies are taken as reference value, and CIS shows a significant overestimation of these energies. However, the true extent of the CIS overestimation is unclear as EOM-CCSD can also suffer from significant errors, and no calculations utilizing the generally accepted "gold standard" ab initio methods, e.g. CC3 or CASPT2, were performed.

Shayan and Nowroozi ${ }^{40}$ also used a combined approach of different single-determinant excited state methods, again using CIS and TD-DFT to study the formation of an intramolecular hydrogen bond in the first excited states of malonaldehyde derivatives. Annaraj et al. ${ }^{41}$ used CIS and TD-DFT to support the experimental observations that the fluorescence of 2-(1-methyl-1H-imidazole-2-carboxamido)-3-phenyl propanoic acid (PAIC) is selectively turned on in the presence of $\mathrm{Ni}^{2+}$. CIS is used to optimize the excited state of the PAIC-Ni ${ }^{2+}$ complex, upon which TD-DFT calculations are run. These calculations demonstrate a significant decrease in the HOMO-LUMO gap of PAIC upon complexation, from $5.56 \mathrm{eV}$ to $2.97 \mathrm{eV}$, which is used as justification for the increase in favorability for fluorescence.

Kocherzhenko et al. ${ }^{42}$ used CIS calculated excitation energies, along with TD-DFT energies, to parametrize an exciton model that can be applied to extremely large chromophore assemblies; here they tackle single, dimer, and hexamer aggregates of the 2-[4-[(E,3E)-3-[3-[(E)-2-[4-[bis[2[tert-butyl-(dimethyl)silyl] oxyethyl]amino]phenyl]ethenyl]-5,5-dimethylcyclohex-2-en-1-ylidene]prop-1-enyl]-3-cyano-5-phenyl-5-(trifluoromethyl) furan-2-lidene]propanedinitrile (YLD124) chromophore. The aim of the study was to parametrize an exciton model for further study of much larger chromophore aggregates, and it was found that CIS, TD-DFT, and the exciton model calculated spectra are all in reasonable agreement.

CIS based methods have also been applied to the absorption behavior of He clusters. Farrokhpur et al. $^{43}$ used CIS and TD-DFT to simulate the absorption of $\mathrm{He}_{\mathrm{n}}$ clusters, where $\mathrm{n}$ ranges from 2 to 29. The authors note that the standard 6-311+G(2d,p) basis set coupled with CIS is insufficient to reliably reproduce the absorption characteristics; instead, it needs to be augmented with further diffuse functions, resulting in a decrease in the CIS error to below $0.5 \mathrm{eV}$. Ge et al. ${ }^{44}$ use a modified version of CIS, i.e. CIS based on absolutely localized molecular orbitals (ALMO), with a spatially localized charge transfer (CT) included in the modified ALMO-CIS formalism. The study predominantly focuses on derivation and implementation of ALMO$\mathrm{CIS}+\mathrm{CT}$, and general qualitative agreement with experimental absorption 
spectra of He clusters, however, it is noted that fine-detail remains elusive. The main advantage for CIS, and its modified variants, in these He cluster situations is that experimental clusters are generally of the size of 1000's of $\mathrm{He}$ atoms, for which higher-level calculations are unfeasible.

Finally, studies have been performed using spin-flip CIS (SFCIS) ${ }^{45}$ and scaled-opposite-spin CIS(D) (SOS-CIS(D)). ${ }^{46}$ Tsuchimochi (2015) extended the SFCIS approach by including exact spin-projection (giving SFPCIS) and included a benchmark of the method against the excited states of some small molecules; namely, $\mathrm{HF}, \mathrm{H}_{3}, \mathrm{~N}_{2}$, and $\mathrm{H}_{4}$. It was found that SFPCIS is capable of qualitatively reproducing conical intersections and general potential energy surface characteristics, as computed using full configuration-interaction (FCI). Chibani et al. ${ }^{47,48}$ and Ponce-Vargas et al. ${ }^{49}$ have applied SOS-CIS(D) to large, highly $\pi$-conjugated boron containing dyes. In these three studies, SOS-CIS(D) energies are used to correct overestimated TD-DFT excitation energies, while keeping several key features that are better captured by TD-DFT; e.g. band shaped of dyes presenting vibronic coupling, solvatochromic effects, CT transfer characteristics, and the impact of auxochromes on the meeting point between absorption and fluorescence curves (AFCP). This hybrid approach is found to decrease the errors in the calculated adiabatic transition energies for the M06-2X functional, however results obtained with the M06 functional are poorer. From this work it was concluded that the M06-2X functional is systematically overestimating the energies, and this systematic error can therefore be reduced; as for the M06 functional, it is unclear as to the origin of the poor agreement.

\subsection{Assessment}

Summing up, the inaccuracies CIS methods are well documented, and compared with multireference and TD-DFT calculations, they have somewhat fallen out of favor (Table 2). Along with TD-HF, they do remain, however, the cheapest possible excited state methods available today, and for extremely large systems, they remain the only viable methods. The simplicity of the methods and their wide-spread implementation means that they are also often included in benchmarking studies, particularly for absorption/emission spectra simulation. Generally, it is found that their accuracy is highly basis set dependent, and the need for large basis sets counteracts their cost advantage. As a result, TD-DFT is generally preferred, although CIS is sometimes more attractive for excited state geometry optimizations for subsequent TD-DFT single points. 
Table 2 Configuration interaction summary.

Property

Trends

Nonlinear optical properties

Absorption spectra

Fluorescence spectra

Photochemical mechanisms

Quantitative photochemical Kinetics

${ }^{a}$ Depends on the system and whether it is a first, second, third order hyperpolarizability, with higher order polarizabilities modelled better than lower order ones.

${ }^{b}$ Depends on the exact method and basis set, with these requiring very large basis sets for accuracy (which in turn defeats its cost advantage).

${ }^{c}$ Due to its low cost, CIS is sometimes used for geometry optimizations in the excited state with TD-DFT improved energies.

\section{Multireference studies}

While HF-based singlet determinant methods, i.e., TD-HF and CIS, have somewhat fallen out of favor due to the general improvement in qualitative studies found when using TD-DFT, the same cannot be said for multireference studies. Multireference methods benefit from being able to fully model regions of electronic degeneracies and can therefore provide accurate information about the shape of excited state potential energy surfaces. As such, they provide mechanistic insights into the photochemical process of choice. In particular, complete active space second order perturbation theory (CASPT2), is often used as the ab initio benchmark, against which the performance of all other computational excited state methods is compared. However, multireference calculations suffer from the exponential scaling of the number of wavefunction configurations that have to be considered with respect to the number of orbitals included in the calculation, limiting these highly accurate methods to systems in which no more than $\sim 20$ orbitals are required to be correlated. ${ }^{50}$ These methods also suffer from non-"black box" behavior; in general, there is no systematic approach to choosing which orbitals are to be included in a multireference calculation. The resulting calculations are therefore dependent on the quality of the nonsystematic starting guess, and therefore the results can suffer. 


\subsection{Mechanistic studies}

The most important use of multireference methods is in clarifying and quantifying photochemical reaction mechanisms, which is often not possible using lower-cost procedures. Below, a number of recent such studies are highlighted.

Sumita and Morihashi ${ }^{51}$ studied the generation of singlet oxygen from an excited thiophene/oxygen exciplex, employing MRMP2-corrected CASSCF geometries. It is found the thiophene undergoes intersystem crossing to a low-lying triplet state after initial excitation, before forming an exciplex with ${ }^{3} \mathrm{O}_{2}$. Further decay, either radiative or non-radiative, takes place to the singlet state, after which either a $[2+4]$ cycloaddition takes place, or ${ }^{1} \mathrm{O}_{2}$ is generated.

Excited state proton transfer of pyruvic acid was studied by Chang et al. ${ }^{52}$ with TD-DFT and CASPT2//CASSCF. The TD-DFT vertical excitation energies are in reasonable agreement with those calculated with CASSCF, and the CASPT2 energy is found to be in excellent agreement with the experimental value. Four nonadiabatic pathways are suggested, and movement along the excited state intramolecular proton transfer coordinate results in a three-way intersection between the $\mathrm{S}_{1} / \mathrm{T}_{1} / \mathrm{S}_{0}$ states. Excited state intramolecular proton transfer of 1-cyclopropyldiazo-2-napthol was studied by Cui et al. ${ }^{53}$ by CASPT2//CASSCF. Their calculations suggest that initial excitation takes place to the $\pi \pi^{*}$ state, from which barrierless radiationless decay can take place to the $\mathrm{S}_{0}$ keto structures, or crossing to the $n \pi^{*}$ state can take place, after which the molecule undergoes decay to the $\mathrm{S}_{0}$ enol structures via a $\mathrm{S}_{1} / \mathrm{S}_{0}$ conical intersection.

Casellas and Reguero ${ }^{54}$ applied a CASPT2//CASSCF approach to study the competition between photosensitization and photocyclization in 9-phenylphenalenone (9-PhPn). It had been found previously that the closely related phenalenone molecule, upon excitation to a low-lying ${ }^{1} \pi \pi^{*}$, undergoes internal conversion followed by efficient intersystem crossing, via a conical intersection, to $a^{3} \pi \pi^{*}$ state. ${ }^{55}$ This triplet state is then available for an efficient energy transfer to form a singlet oxygen species with a quantum yield of 0.95 . In contrast, 9-PhPn exhibits a quantum yield of merely $0.08 .^{56}$ The authors employed CASSCF optimised geometries upon which improved CASPT2 single-point energies were calculated. It was shown that the decrease in quantum yield is due to the photocyclization reaction that can take place between the neighboring phenyl and ketone groups. As with the original study, the CASPT2-corrected CASSCF geometries are 
generally found to be sufficiently accurate to provide insight into the observed processes. However, the critical minimum energy geometries of the ${ }^{1} \pi \pi^{*}$ state, as calculated individually at the CASSCF and CASPT2 levels of theory, have drastically different structures. The equilibrium structure from CASSCF has a phenyl group almost perpendicular to the rest of the phenalenone structure, but the CASPT2 structure has these two groups almost planar to each other. This has important consequences in the ensuing cyclization reaction, in which the close vicinity of the two reacting groups is necessary; in this respect, it is a failure of the CASSCF method.

Casellas et al. ${ }^{57,58}$ have also applied the CASPT2/CASSCF approach to studying the photoisomerization reactions of azobenzene and phenylazopyridine. Given the planar structure of the $\mathrm{N}=\mathrm{N}$ double bond, it is possible for the isomerization to take place via either a rotational or non-rotational pathway; for both azobenzene and phenylazopyridine, the rotational pathway is favored thermally. On the excited states, however, the potential energy surface shows that both pathways become competitive.

Dai et al. ${ }^{59}$ have employed CASPT2 corrected CASSCF potential energy surfaces to explore the photolysis of the carbon-iodine bond found in iodouracil, finding intersystem crossing and conical intersections are involved in converting the bright $\pi \pi^{*}$ to dissociative $\pi \sigma^{*}$ and $n \sigma^{*}$ states. The calculated vertical excitation energies for the first 5 electronic states of iodouracil are found to be in good agreement with the experimental UV-vis absorption, with the calculations showing the lowest excited state is in fact a dark $\pi \sigma^{*}$ transition, followed by the bright $\pi \pi^{*}$ transition.

Another recent study by Menzel et al. ${ }^{7}$ utilizes CASSCF calculations to explore the photoreactivity of two ligation reactions; i.e., photoactivation of o-methylbenzaldehyde to form a reactive o-quinodimethane, via a conical intersection, and the photolysis of 2,5-diphenyltetrazole, in which conical intersections also play a profound role. In both systems, CASSCF potential energy surfaces (PES) were found to be sufficient to describe the geometries at which electronic degeneracies were found. As well, the study also employed TD-DFT to provide qualitative insight into the nature of the first few low-lying excited states. While TD-DFT is incapable of fully describing the conical intersections involved, it is sufficient in describing the symmetry forbidden, and therefore spectroscopically dark, $n \pi^{*}$ transition. It is this transition that is responsible for the observed peak in per-photon reactivity taking place at a lower wavelength than the absorption maxima.

From an experimental perspective, it is interesting to compare this wavelength dependence to the study by Dai et al., as in the latter study the 
reactivity is directly reconciled by the experimentally observed absorption spectrum of iodouracil; i.e., the higher energy, bright $\pi \pi^{*}$ state is initially populated and is the origin of the resulting photochemistry. However, Menzel et al. demonstrate that absorption efficiency does not necessarily correspond to photochemical efficiency, and that from a per-photon perspective, it can be much more useful to directly aim for the dark states. Certainly, from the PES shown by Dai et al., directly hitting the lower energy $\pi \sigma^{*}$ state should directly result in the $\mathrm{C}-\mathrm{I}$ bond photolysis and could be more efficient as competition from higher energy pathways may no longer be relevant.

Yuan and Bernstein et al. have also performed several number of multireference studies in the past few years, applying CASSCF calculations to the excited state decomposition reactions of $3,3^{\prime}$-diamino-4, $4^{\prime}$-bisfuroxan and $4,4^{\prime}$-diamino- $3,3^{\prime}$-bisfuroxan, ${ }^{60}$ bis(ammonium) $5,5^{\prime}$-bistetrazolate and bis(triaminoguanidinium) $5,5^{\prime}$-azotetrazolate, ${ }^{61}$ dihydroxylammonium $5,5^{\prime}$ bistetrazole-1, $1^{\prime}$-diolate and dihydroxylammonium $3,3^{\prime}$-dinitro-5, $5^{\prime}$-bis1,2,4-triazole-1,1' ${ }^{\prime}$-diol, ${ }^{62}$ nitrophenyl derivatives, ${ }^{63}$ and 1,1-diamino-2,2dinitroethylene, ${ }^{64}$ as well as the fragmentation of excited state ammonia-based clusters. ${ }^{65}$ In the five decomposition studies, the products are a ring-opened tetra/triazoles, $\mathrm{NO}$ and/or $\mathrm{N}_{2}$. In particular, emphasis is placed on the role of conical intersections in the ring opening/decomposition reactions; generally, neither the ground state nor excited state PES are dissociative, however, the conical intersections found between the two states are accessible and result in a vibrationally hot molecule with sufficient energy to undergo homolysis. In the case of the bisfuroxan derivatives, it is found that MP2-corrected CASSCF calculations are required to produce a PES capable of reconciling experimental observations.

A CASPT2//CASSCF study by Datar and Hazras ${ }^{66}$ examined the two possible nonradiative decay pathways of 5,6-dihydroxyindole. Upon excitation and internal conversion, it is found that there are two conical intersections between the low-lying $\pi \sigma^{*}$ and $\mathrm{S}_{0}$ states, along both an $\mathrm{OH}$ and the $\mathrm{NH}$ bond stretching coordinates. The barrier to the conical intersection located along the $\mathrm{OH}$ coordinate is lower than the corresponding barrier along the NH coordinate. Once over the barriers, it is possible not only to decay to the ground state, but also to continue to dissociation to form a dihydroxyindole radical and free hydrogen atom. However, it is noted that nonadiabatic dynamics simulations are required to fully determine the extent of the competition between the two pathways. Kobayashi et al. ${ }^{67}$ employed extended multistate (XMS-) CASPT2 and ultrafast visible and infrared 
spectroscopy to probe the dynamics of the delocalized biradical excited form of pentaarylbiimidazole. The ultrafast spectroscopy revealed the existence of two transient isomers, each with a differing amount of biradical character. XMS-CASPT2 calculations demonstrate the metastable isomers are likely stabilized by the pi-stacking present between the imidazole rings present.

Kim et al. ${ }^{68}$ employed CASSCF, DFT, and CC methods to study the photoisomerization of 1,2-dihydro-1,2-azaborine, in which the planar, six-membered ring can isomerize to a Dewar product by two possible pathways. The study finds that one of the transition states is greatly reduced on the $\mathrm{S}_{1}$ state, and the molecule can decay to the $\mathrm{S}_{0}$ state by a conical intersection to form a stable intermediate product. This product is then able to further isomerize either to the Dewar product or back to the planar, six-membered ring. Gao et al. ${ }^{69}$ studied the photoisomerization of $1,1^{\prime}$-dimethyl-2,2'-pyridocyanine, using CASPT2//CASSCF energies. The isomerization takes place on the $\pi \pi^{*}$, on which it is barrierless, before decaying to the $\mathrm{S}_{0}$ state via a conical intersection. Lei et l. $^{70}$ studied the photoisomerization of stilbene with CASSCF, finding four left-hand and four right-hand conical intersections between the $\mathrm{S}_{0}$ and $\mathrm{S}_{1}$ states.

Suaud et al. ${ }^{71}$ used CASSCF to fully correlate the $\pi$-electrons of some conjugated, mono- and di-radical hydrocarbons, against which the delocalization of the radicals as calculated with ROHF is compared. It is found that the SOMO as calculated with ROHF are generally much more localized than as calculated with CASSCF, and that increasing the size of the active space also results in an increase in delocalization. Zhang et al. ${ }^{72}$ used CASSCF to gain insight into the electronic spectra of linear carbene chains $\mathrm{H}_{2} \mathrm{C}_{\mathrm{n}}$, where $n=3-10$. The study shows that chains with even values of $\mathrm{n}$ are more stable than those where $\mathrm{n}=\mathrm{odd}$, and that there is a nonlinear relationship between chain length and vertical excitation energy. Hlavacek et al. $^{73}$ studied the $\pi$-conjugated acrolein molecule with CASPT2 and $\operatorname{CCSD}(\mathrm{T})$, in order to determine structural properties of the its $\mathrm{T}_{1}$ state. The methods are used to simulate cavity ringdown $(\mathrm{CRD})^{74}$ spectra, finding good agreement between the experimentally obtained and simulated spectra. No comparison with other, lower levels of theory is included, however. Li et al. ${ }^{75,76}$ compared CASSCF/CASPT2 with CASPT2-corrected SF-TD-DFT for the photoisomerization of 9-(2,4,7-trimethyl-2,3dihydro-1H-inden-1-ylidene)-9H-fluorene, an example of a 2nd generation rotary molecular motor. The results show the importance of the conical intersections that lead the cis-stable to the trans-unstable isomer upon excitation and find the TD-DFT-based methods can capture this behavior. 
However, the TD-DFT based geometries are generally lower in energy than the multireference energies, but the structural parameters of the conical intersection geometries are in close agreement. Amatatsu has also performed CASSCF calculations on 9-(5-methyl-2-phenyl-2-cyclopenten-1-ylidene)$9 \mathrm{H}$-fluorene (MPCPF) and various derivatives, ${ }^{77-81}$ showing initially that the design of molecular rotary motors should include groups with internal vibrations that are strongly coupled with the bridging carbon-carbon double bond. The later investigations more fully characterize the conical intersections along the isomerization pathway.

The excitation energy transfer mechanism in a peridinin-chlorophyll $a$ complex has been studied by Bricker and Lo. ${ }^{82,83}$ Here, the low-lying excited states of the complex are calculated using CASSCF, and the corresponding transition dipole moments were used to determine the Coulombic couplings between the two species within the complex. It was found that there is significant coupling between not only the $\mathrm{S}_{1}$ and $\mathrm{Q}_{\gamma}$ states of the peridinin and chlorophyll $a$, respectively, but also between the $S_{2}$ and $\mathrm{Q}_{x / y}$ states. It is this extra coupling, that explains the experimentally observed, highly-efficient energy transfer.

Further multireference investigations into photomechanisms of organic molecules include a study by Guan et al. ${ }^{84}$ who used CASPT2//CASSCF and TD-DFT energies to study the photochemistry of Sudan I and Orange II dyes, employing an azobenzene parent structure that is present in both sets of molecules as the computational model. It was found that initial excitation of the azobenzene structure is to the $\mathrm{S}_{2}, \pi \pi^{*}$ state, from which barrierless decay can take place along either the $\pi \pi^{*}$ state to the keto $\mathrm{S}_{0}$ state, via a $\mathrm{S}_{2} / \mathrm{S}_{0}$ conical intersection, or crossing to the $n \pi^{*}$ state can take place to either the cis- or trans-enol structures. Another study employed CASSCF/CASPT2 to assess the two possible elimination reactions of 6,7 -dihydro- $5^{4}$-benzo[d]pyrido [2,1-f]azaborininr; the two eliminations either produce $\mathrm{H}_{2}$, or $\mathrm{BH}_{3}$ and a corresponding ring contraction. On the ground state, the ring contraction mechanism is found to be thermally favorable; the reaction barrier for $\mathrm{H}_{2}$ production is $\sim 43 \mathrm{~kJ} \mathrm{~mol}^{-1}$ higher in energy. However, on the excited state it is found the $\mathrm{PES}$ for $\mathrm{H}_{2}$ production is essentially barrierless, with a reaction barrier for ring contraction increasing from $+233.5 \mathrm{~kJ} \mathrm{~mol}^{-1}$ on the $\mathrm{S}_{0}$ state to $+252.8 \mathrm{~kJ} \mathrm{~mol}^{-1}$ (from the Franck-Condon point) on the first excited state. There are also several conical intersection geometries found, between the $S_{0}$ and $\mathrm{S}_{1}$ states, with the two intersections on the $\mathrm{H}_{2}$ pathway either taking the molecule to the original equilibrium geometry, or to the $\mathrm{H}_{2}$ product on the ground state. 


\subsection{Restricted active spaces (RAS)}

Although multireference methods suffer from exponential scaling issues with respect to the number of orbitals included in the calculations, they can still be applied to large systems with some success. Ben Amor et al. ${ }^{77}$ employed CASPT2 to study the valence excitations of an iron-porphyrin complex. In this complex, if all possible $\pi / \pi^{*}$ and metallic $3 \mathrm{~d}$ orbitals were included, an active space of 36 electrons in 36 orbitals would be required, far beyond current computational capabilities. Instead, a truncated active space is used employing the $3 d_{n}$ orbitals of the iron and $6 \sigma\left(^{*}\right)$ and $\pi\left(^{*}\right)$ orbitals that exhibit significant changes in occupancy upon excitation. Also, only the excited states up to approximately $3 \mathrm{eV}$ are investigated, above this point (the so-called Soret Band), excitations are dominated by $\pi \pi^{*}$ transitions and would require their inclusion in the active space. The difficulties in determining an appropriate active space and the expense of the resulting calculations may warrant the usage of a cheaper method, for example TD-DFT. However, CASPT2 results reveal the presence of not only singly excited states, generally dominated by $d_{n} \pi^{*}$ transitions, but also low-lying doubly excited states; states that are necessarily not included in TD-DFT calculations.

Venturinelli et al. ${ }^{78}$ used CAS- and RAS- procedures to study the spin states of manganese-porphyrin complexes. Like Ben Amor et al., the Mn-Por complexes also require an extremely large active space to fully accommodate the relevant $\mathrm{d}, \sigma$, and $\pi$ orbitals; however, partitioning of the active space in order to fully and partially correlate different orbitals, as is the case with RASSCF and RASPT2, allows for a larger number of orbitals and electrons to be included than would otherwise be possible. In their study, Venturinelli et al. were able to correlate 28 electrons in 28-30 orbitals using a RAS-procedure, significantly more than the 14 electrons in 16-18 orbitals that were able to be correlated in the accompanying CAS calculations. The calculations demonstrate the existence of low-lying, high-spin configurations of the Mn-porphyrin complexes, which have significant implications for the catalytic activity of such species. Bai et al. ${ }^{79}$ employed CASSCF, along with a number of other excited state methods, to study a ruffled porphyrin species. The active space used was, however, reasonably small (6 electrons, 6 orbitals), with little emphasis placed on the CASSCF results. Crossing points were located, however, between the $T_{1}$ and $S_{0}$ states, and presented as a possible route for excited state energetic decay.

The excited states of nucleobases have been studied by Segarra-Martí et al., ${ }^{80,81,85-87}$ with CASSCF and RASSCF, and their corresponding perturbation theory CASPT2 and RASPT2 counterparts. In the study of 
adenine alone, ${ }^{80}$ its high energy electronic states are investigated by RAScalculations, as this allows a larger active space, and therefore higher energy virtual- and lower energy occupied-orbitals, to be considered. Interestingly, the nonlinear optical properties of adenine were investigated, as this allows the modelling of pump-probe experiments to be performed accurately. The third-order nonlinear properties of cytosine, thymine, and uracil were then studied, ${ }^{86}$ finding that the resulting two-dimensional spectra are highly sensitive to small structural changes, especially important when trying to differentiate between the similar thymine and uracil nucleobases.

CASPT2//CASSCF studies were then performed on thymine to study the possible photodeactivation pathways that are available upon excitation to the low-lying, bright $\pi \pi^{*}$ state. It was found that the CASSCF and CASPT2 relaxed geometries for this state are similar, however CASPT2 points to a twisted geometry, compared with a planar structure predicted by CASSCF, and that radiationless decay takes places via a $\pi \pi^{*} / \mathrm{S}_{0}$ conical intersection. As well as this, the CASSCF PES was found to be overly steep compared with that of CASPT2, and this difference would likely lead to inaccurate dynamic simulations, as needed for quantitative analysis of the excited state lifetimes. Finally, the dissociative electron detachment (DEA) reactions of the four DNA nucleobases was studied, again with CASPT2//CASSCF energies. For each of the four nucleobases, each possible $\mathrm{N}-\mathrm{H}$ homolysis reaction was modelled. For each $\mathrm{N}-\mathrm{H}$ coordinate, the PES of the anionic $\pi \pi^{*}-$ and dissociative $\pi \sigma^{*}-$, and neutral $\mathrm{S}_{0}$ are calculated, and generally intersections are found between the bright $\pi \pi^{*}-$ and dark $\pi \sigma^{*}-$, that will result in hydrogen ejection. The relative energies of these intersections are used to determine the contributions of the respective dissociation channels.

Santolini et al. ${ }^{88}$ used CASSCF and RASSCF to study the photochemical reactions of cis-butadiene and cyclopentadiene. It was found that small CASSCF active spaces result in a poor balance of covalent and ionic valence bond (VB) structures, and an extended active space is therefore required for accurate potential energy surfaces. To that end, RASSCF is a natural choice for this active space extension. Calculations of vertical excitation energies demonstrate the failing of CASSCF to correctly predict the ordering of the first two excited states for both cis-butadiene and cyclopentadiene, whereas the larger RAS calculations correctly predict the ordering and are closer to experimental values of excitation energy. Xia et al. ${ }^{89}$ studied the ring-opening of cyclopropenone, cyclobutanone, cyclopentanone, and cyclohexanone. The authors use CASPT2-corrected CASSCF 
structures to compute the PES, finding that the excited state pathway to the $\mathrm{S}_{0}$ ring-opened isomers is via a $\mathrm{S}_{1} / \mathrm{S}_{0}$ conical intersection in all four molecules. Increasing ring strain is also found to decrease the barrier to the conical intersection geometry. Bera et al. ${ }^{90-92}$ studied the excited state decomposition reactions of dimethylnitramine and corresponding clusters with $\mathrm{Fe}, \mathrm{Zn}$, and $\mathrm{Al}$ with CASSCF and RASSCF calculations. The calculations were able to find a variety of conical intersections between the relevant electronic excited states and determine which are dissociative and result in decomposition.

\subsection{Quantum Monte-Carlo (QMC)}

Manni et al. ${ }^{93}$ employed (metallo-) porphyrins as a difficult test case for multireference calculations, to test the performance of their newly developed full configuration interaction quantum Monte Carlo (FCIQMC) code for solving the CASSCF equations. Here, instead of a deterministic approach to fully correlating a subset of orbitals, a QMC approach is employed in which the configuration state functions are sampled stochastically. The authors find good agreement with experimentally obtained data for the absorption and ground state properties of the porphyrins studied. Further investigations are required, however, to determine the impact of including dynamic correlation corrections to the obtained wavefunctions.

Asturiol and Barbatti ${ }^{94}$ studied the examined the formation of ${ }^{1} \mathrm{O}_{2}$ upon encountering excited porphyrin. It is found that efficient intersystem crossing will predominantly yield triplet porphyrin, which leads to inefficient generation of singlet oxygen. However, formation of ${ }^{1} \mathrm{O}_{2}$ is efficient if singlet excited porphyrin is encountered. Kornobis et al. ${ }^{95}$ used TD-DFT with a range of functionals, EOM-CCSD, and MC-XQDPT2-corrected CASSCF energies, to study the $\mathrm{S}_{1}$ state of $\mathrm{Co}^{3+}$-corrinoids. Previous literature had described the $S_{1}$ state as either a $\pi \pi^{*} \operatorname{transition}^{96,97}$ or a metal to ligand charge transfer transition, ${ }^{98-100}$ and this study aimed to unambiguously determine the dominant character of the $\mathrm{S}_{1}$ state, finding with the MC-XQDPT2 calculations that it is, in fact, dominated by MLCT character. It is found that the B3LYP, mPW1PW91, and TPSS-h functionals incorrectly predict a $\pi \pi^{*}$ state, whereas EOM-CCSD, and the BP86, BLYP, MPWPW91, and LC-BLYP functionals correctly predict metal to ligand charge transfer dominant character.

Rotllant et al. ${ }^{101}$ studied the photochemistry of acetophenone using CASSCF and XMCQDPT2. The equilibrium geometries of the low-lying, singlet and triplet $\pi \pi^{*}$ and $n \pi$ states are optimised at both levels of theory, 
and the carbon-oxygen bond stretch is found to be a convenient reaction coordinate. The efficient crossing from the ${ }^{1} n \pi^{*}$ is thought to take place via a three-state crossing point between the ${ }^{1} n \pi^{*},{ }^{3} n \pi^{*}$, and ${ }^{1} \pi \pi^{*}$ states. It is also found that CASSCF generally underestimates the length of the carbon-oxygen bond for each of the equilibrium structures, highlighting the importance of including dynamic correlation in the calculations.

In other studies, $\mathrm{Xu}$ et al. ${ }^{102}$ proposed a diabatization scheme, and applied it to the dissociation of phenol. Energies were calculated using MC-QDPT2 and coupling modes at the conical intersections between the $\pi \pi^{*} / \pi \sigma^{*}$ and $\pi \pi / \pi \sigma^{*}$ states were calculated. As the algorithm was shown to give good, smooth results, it was proposed that it can therefore be used in dynamic simulations. Kletskii et al. ${ }^{103}$ studied the competition between Norrish-type reactions in butanal, pentanal, penta-2-one, buten2-al, butanimine-1, and nitrosopropane with CASSCF and XMCQDPT2. For each molecule, the possible dissociation and $\mathrm{H}$-transfer pathways were studied in order to determine their relative competition, finding that increasing alkyl chain length had limited effect on the Norrish-I reaction pathway, but decreased the energy of the $\mathrm{H}$-transfer reactions.

\subsection{Benchmarking studies}

In addition to studying photochemical reactivity, multireference methods of various flavors are also commonly used to test the accuracy of lower-cost methods such as TD-DFT, or indeed lower-cost multireference methods. Retinal, and various derivatives and truncated models of it, have been employed in recent years as a system against which a wide-range of methods can be tested and benchmarked. Andruniów et al. ${ }^{104-106}$ have employed CASPT2, CASPT3, CASSCF, CASPT2/CASSCF, DFT, MP2, (L)CC2, and $\operatorname{CCSD}(\mathrm{T})$ to test the accuracy of optimised geometries, and vertical and adiabatic excitation energies; CASPT2 energies/geometries are taken as the benchmark. For the study including 9 retinal derivatives, ${ }^{105}$ it was generally found that methods that include dynamic correlation (i.e., not CAS$\mathrm{SCF}$ ), gave ground state geometries in good agreement with those of CASPT2, with CASSCF giving significant differences in bond lengths, and in two retinal models, significantly twisted structures that are planar by the other methods. CASSCF was also found to consistently overestimate vertical excitation energies, however, the CASPT2 vertical excitation energies on CASSCF geometries were found to be accurate, provided the CASSCF geometry resembles the equivalent CASPT2 geometry. 
A smaller retinal model was used to test the CASPT3 method, ${ }^{104}$ finding that it performed exceptionally well for ground state geometries, was insensitive to IPEA shifts, but more sensitive to basis set size than CASPT2. A further three retinal models were employed to test CASPT2/CASSCF, CASPT2, and CC2. ${ }^{106}$ It was found that CASSCF is incapable of locating the planar $\mathrm{S}_{1}$ minimum geometry structures for four out of the five studied retinol models. CC2, on the other hand, was as successful as CASPT2 in locating these minimum energy geometries. However, when CASSCF finds the correct structure, the one-electron properties, emission, and adiabatic energies are in excellent agreement with those of CASPT2. When extended to the $\mathrm{S}_{2}$ states, which are found to have significant multireference/double excitation character, CC2 is found to give transition energy errors of $>1 \mathrm{eV}$. Notably there does not seem to be any clear indicators of when CASSCF geometries will fail, in which case CASPT2 corrections will not result in any improvements in mechanistic accuracy. This is, thus, especially troublesome in larger molecules, when running CASPT2 geometry optimizations may not be feasible due to their computational expense.

Further benchmarking studies with retinal models have been performed by Olivucci et al. ${ }^{107-114}$ The original study ${ }^{107}$ employed CASSCF and multireference methods that capture dynamic electron correlation (MRCISD, CASPT2, NEVPT2, and XMCQDPT2) to explore the ground state PES of a retinal model. As with the studies by Andruniów et al., it is found that the CASSCF geometries, and resulting PES, are liable to failure, and the additional inclusion of dynamic correlation results in a significant reshaping of the PES. Subsequent studies employed TD-DFT, ${ }^{108,109}$ coupled-cluster based methods, ${ }^{110-112}$ quantum Monte Carlo, ${ }^{113}$ and ADC(2) and ADC(3) methods, ${ }^{114}$ which were generally compared with the high-level MRCISD(+Q) PES. Generally, most methods were capable of qualitatively reproducing the PES from high-level calculations, with the EOM-CC methods seemingly capable of reproducing key photochemical pathways, with EOM-SF-CCSD $(\mathrm{dT})$ shown to be capable of quantitatively describing some photoisomerization pathways.

TD-DFT and CASSCF/CASPT2 calculations were applied to the pyrene and its 3 possible protonated forms by Chin and Lin, ${ }^{115}$ from which absorption and fluorescence spectra were calculated. The calculated vertical excitation energies calculated by TD-B3LYP and CASPT2 are in close agreement for the four pyrene species, however, the size of the basis set, i.e., cc-pVDZ, is reasonably small. Cacelli et al. ${ }^{116}$ employed TD-DFT and multireference calculations to study the absorption behavior of anthocyanidins, 
again finding reasonable agreement between DFT and MRPT2 vertical excitation energies, with B3LYP finding the closest agreement. However, the vertical excitation energies were calculated for DFT equilibrium geometries, with no comparison made between DFT and MRPT2 structural parameters. Were these to be included, the vertical excitation energies may be significantly different.

The competition between photoisomerization and photodegradation mechanisms in the first excited state of tetrazoline oxime ether has been studied with TD-DFT and CASSCF by Fréneau et al. ${ }^{17,118}$ The calculations show that the photodegradation pathway is favored in the $E$-isomer, whereas photoisomerization is favored in the $Z$-isomer; this is due to the hydrogenbond present in the Z-isomer that prevents that photolysis pathway from being favored. López-de-Luzuriaga et al. ${ }^{119}$ used TD-DFT and CASSCF/ CASPT2 to study the dual-fluorescence of 4-(dimethylamino)-pyridine in different solvent environments, finding good agreement between the two methods and experimental data. Li et al. ${ }^{120}$ used TD-B3LYP and CASPT2/CASSCF energies to study the low-lying excited states of coumarin, finding good agreement between both methods and experimental data.

Olsen ${ }^{121}$ employed CASSCF to study the competition between the charge-transfer (CT) and locally excited (LE) states of green fluorescent protein (GFP) derivatives, finding that strongly electron donating groups enhance CT character in the $\mathrm{S}_{1}$ state, compared with weakly donating/ electron withdrawing groups. Ai et al. ${ }^{122}$ used TD-DFT and CASSCF to study the one- and two-photon absorption of GFP. CASSCF is found to give results in good agreement with experimental data, whereas CAM-B3LYP is found to be in poor agreement. The blue-shift observed was found to be due to the vibronic coupling present through a carboncarbon-carbon single, double bond moiety.

In other studies, Zhang et al. ${ }^{123}$ applied TD-B3LYP and CASPT2 to study substituent effects on the absorption properties of phenol radicals, finding good agreement between the TD-DFT, CASPT2, experimental absorption spectra. Palmer et al. have recently performed a number of multireference studies on several organo-halide molecules; namely, iodopentafluorobenzene, ${ }^{124,125}$ difluoromethane, ${ }^{126}$ pyridineN-oxide, ${ }^{127}$ iodobenzene, ${ }^{128}$ and chlorobenzene. ${ }^{129}$ Each study used both single- and multireference calculations, and generally finds good agreement between both types of calculations and experimental data, for both adiabatic and vertical excitation energies. 


\subsection{Assessment}

To summarize, multireference methods have been applied to a wide range of molecules, from some as large as porphyrin to those as small as hydrogen. Applied properly, they offer high degrees of accuracy but at the high computational expense (Fig. 2). In particular, multireference calculations provide accurate insight into the photochemical processes being investigated, against which less accurate, but cheaper, methods are compared; most notably, TD-DFT. Multireference calculations continue to be successfully applied to systems in which nonadiabatic behavior plays an important role in the photochemical process of interest. A particularly popular approach involves using CASSCF calculated geometries, upon which potential energies are improved by performing MRPT2 single-point calculations. However, it has been shown that the neglect of dynamic correlation in CASSCF can result in incorrect geometry minima, which can have a profound effect when attempting to reconcile observed photochemical behavior. These spurious geometries can be unpredictable, and so it can be difficult to ensure that truly correct behavior is being simulated if CASPT2 calculations are not feasible. The methods also suffer from active orbital choice, also has a profound impact on the success or failure of these methods, though the careful use of restricted active space methods and quantum Monte-Carlo methods can effectively address that problem.

\begin{tabular}{|c|c|}
\hline \multicolumn{2}{|c|}{ Multi-reference methods } \\
\hline Advantages & Disadvantages \\
\hline $\begin{array}{l}\text { Highly Accurate } \\
\text { - sometimes the only } \\
\text { accurate approach available } \\
\text { e.g. for non-adiabatic } \\
\text { surfaces, many other } \\
\text { photochemical reactions }\end{array}$ & $\begin{array}{l}\text { High computational cost } \\
\text { made worse by need for } \\
\text { - large active spaces RAS- or } \\
\text { QMC methods can be used } \\
\text { to expand active space at } \\
\text { lower cost }\end{array}$ \\
\hline $\begin{array}{l}\text { Main Applications } \\
\text { - photochemical reaction } \\
\text { mechanisms } \\
\text { - excited state optimizations } \\
\text { - benchmarking lower-cost } \\
\text { levels of theory }\end{array}$ & $\begin{array}{l}\text { dynamic correlation } \\
\text { CASPT2//CASSCF often } \\
\text { used to improve efficiency } \\
\text { but some CASSCF } \\
\text { geometries are inaccurate } \\
\text { and these are hard to } \\
\text { anticipate }\end{array}$ \\
\hline
\end{tabular}

Fig. 2 Main advantages and disadvantages of multireference studies. 


\section{Time-dependent density functional theory}

TD-DFT and its counterpart that employs the Tamm-Dancoff approximation (TDA-TD-DFT) ${ }^{130}$ are hugely popular excited state methods that are largely analogous to TD-HF and CIS, respectively, but with the inherent advantage of the included dynamic correlation present in DFT. The methods benefit from the cost-effectiveness of DFT and can therefore be applied to a huge range of molecules that are simply not feasible for ab-initio methods, providing qualitative and, sometimes, quantitative insight into photochemical processes. However, as with ground state DFT studies, the accuracy of the calculations is dependent on the functional of choice, which can provide wildly different values for identical calculations. TD-DFT will suffer when excitations include charge-transfer, Rydberg character, or when the excited state wavefunction requires anything other than a single-determinant description (regions of degeneracy, double-excitations). Due to the large volume of literature that include TD-DFT calculations of some description, this section will concentrate on some of the most highly cited studies in the 2013-2018 period.

\subsection{Methodological developments}

\subsubsection{Long-range corrected functionals}

One of the key decisions in choosing a functional is whether or not it is long range corrected (LC). LC functionals treat electron-electron exchange using $\mathrm{HF}$ at large separation and using a generalized gradient exchange (GGA) at short range and have been shown to have improved accuracy for states with significant charge transfer character. ${ }^{131-133}$ The performance of LC functionals depends on the choice of the range separation parameter $(\omega)$ that determines the balance of GGA and HF exchange at intermediate interelectronic distance. ${ }^{134-139}$ While the parameter can be used ad hoc to fit predictions to experimental data, a more "a priori" approach has been advocated by Baer in which the parameter is tuned to make the negative of the highest occupied molecular orbital (HOMO) equal to its ionization potential in accordance with Kohn-Sham theory.

Gasiorski et al. ${ }^{144,145}$ employed a modified form of the B3LYP, CAMB3LYP, and LRC-BLYP functionals, for which the exchange screening parameter, $\omega$, was varied, in order to study the absorption and fluorescence properties of 1,3-diphenyl-1H-pyrazolo[3,4-b]quinoxalines. The study finds that the long-range corrected functionals give absorption/emission 
in closer agreement than the uncorrected B3LYP functional. However, this good agreement could be the result of fitting $\omega$ to the data. No comment is made on the nature of the excited state, and whether this seems to be functional dependent.

Arulmozhiraja and Coote ${ }^{146}$ used four long-range corrected functionals (CAM-B3LYP, $\omega-B 97 X-D$, LC-BLYP, and LC- $\omega$ PBE), along with standard hybrid and double hybrid functionals, in an attempt to describe the excited states of indole and azaindole. The long-range corrections were determined a priori using Baer's method. It was found that while some excited properties were improved, others were not and no one functional predicted the correct order of all of the excited states for both species.

In a similar vein, Eriksen et al. ${ }^{147}$ discuss in detail the failure of CAMB3LYP to correctly describe the charge-transfer character of the $\mathrm{S}_{1}, \pi \pi^{*}$ transition of para-nitroaniline. In particular, compared to CCSD and SOPPA calculations, CAM-B3LYP is found to not only be unable to reproduce experimental gas-phase absorption spectra, but also fails in describing the bathochromic shift that is also observed experimentally; this is a worrying failure as, the authors note, the CAM-B3LYP functional is designed to correct for the failings of B3LYP when modeling charge transfer in molecules. The study also includes other hybrid functionals, for example, BHHLYP, as well as CIS/RPA, finding that the errors are even more pronounced. The incorrect energies are found to arise from an overestimation of charge-transfer character in the ground state, apparent through a calculated dipole moment that is larger with CAM-B3LYP than with CCSD, and a resulting underestimation of CT in the $S_{1}$ state. This effect is found to become more pronounced in the solvent calculations; the authors also examine the contribution of the solvent field to the excitation energies, finding similar contributions for both TD-DFT and CCSD calculations, suggesting the solvent model is not to blame. Finally, the authors find that the difference in TD-DFT and TDA-TD-DFT energies can be used as a measure for the extent of CT.

Körzdörfer and Brédas ${ }^{148}$ also note that the failings of semilocal and global hybrid functionals can be attributed to the error associated with electron delocalization inherent in such functionals. However, the authors show that tuning the $\omega$ value of the $\mathrm{LC}-\omega \mathrm{PBE}$ against the ionization potential of the molecules of interest can reduce ensuing error in ensuing TD-DFT calculations. Similarly, Sun et al. ${ }^{149}$ employ a set of organic emitters that undergo thermally activated delayed fluorescence (TADF), which represent a challenge for TD-DFT with traditional functionals. In particular, the authors 
note that the organic emitters exhibit donor-acceptor behavior and therefore they tune the $\omega$ value against both ionisation potential and electron affinity. The studies show that the mean absolute deviation and maximum absolute error for the tuned functional are greatly reduced, particularly for the adiabatic singlet-triplet energy gaps. Sun et al also, however, continue to find good performance of the B3LYP and M06- $2 \times$ functionals for the properties investigated, however, this is attributed to favorable error cancellation rather than proper treatment of physical phenomena.

Penfold $^{150}$ also tunes the range-separated parameter associated with the LC-BLYP functional against both ionization potential and electron affinity of TADF emitters. Although Penfold employs a slightly different formalism in terms of optimization procedure, like Sun et al., Penfold finds that such an a priori tuning reduces errors associated with vertical ionization energy and the singlet-triplet energy gaps that are important when considering TADF candidates. Penfold also notes that the spin orbit coupling between the singlet and triplet states are not considered in his work, which are also important properties to consider as they determine the efficiency of any possible state-crossing processes.

Moore and Autschbach ${ }^{151}$ evaluated the performance of TD-DFT (BP, $\mathrm{PBE}, \mathrm{PBE} 0, \mathrm{LC}^{-} \mathrm{PBE}^{*}, \mathrm{LC}-\mathrm{PBE} 0^{*}$ ) in describing the excited states of linear cyanines. The study finds that, unlike with charge transfer states, minimizing the delocalization error $(\Delta \mathrm{E})$, for example, by tuning the range-separated functionals, does not lead to a concomitant increase in accuracy in absorption maxima. It is also found that local pure functionals overestimate vertical excitation energy, and a $\triangle$ SCF approach significantly underestimates the vertical excitation energy. TD-DFT in general is also found to overestimate singlet-triplet energy gaps.

\subsubsection{Relaxed versus unrelaxed densities}

Maschietto et al. ${ }^{152}$ have compared the effect of using relaxed or unrelaxed excited state density when attempting to describe the nature and properties of excited states. Excited state charge-transfer characteristics of extended phenyl chains are examined by computing $\mathrm{D}_{\mathrm{CT}}$ values for each molecule, using either relaxed $\left({ }^{\mathrm{R}} \mathrm{D}_{\mathrm{CT}}\right)$ or unrelaxed $\left({ }^{\mathrm{U}_{\mathrm{D}}} \mathrm{CT}\right)$ excited state densities. These values correspond to the spatial extent of the charge transfer upon excitation and the charge transfer distances, respectively. In total, 52 exchange correlation functionals were employed in order to determine their performances. Generally, it was found that the description of the CT state was qualitatively described by both ${ }^{\mathrm{R}} \mathrm{D}_{\mathrm{CT}}$ and ${ }^{\mathrm{U}_{\mathrm{D}}} \mathrm{D}_{\mathrm{CT}}$ values, and that as charge-transfer increased in distance, the use of $\mathrm{U}_{\mathrm{CT}}$ becomes more 
appropriate. The performance of the CT description was found to be relatively functional independent, and much more dependent on the extent of the CT instead.

\subsubsection{Dual-functional Tamm-Dancoff approximation}

Shu, Parker, and Truhlar ${ }^{153}$ report a new implementation of TD-DFT, i.e., Dual-Functional Tamm-Dancoff Approximation (DF-TDA), that should correctly reproduce the $3 N-8$, where $N$ is the number of atoms, dimensionality that describes conical intersections. The proposed implementation uses two DFT functionals; the first functional is used to optimize the orbitals of the system, and the second functional is used to construct the TD-DFT/TDA Hamiltonian; the method is implemented in GAMESS. In order to assess the performance of the method, combinations of the M06, M11, and MN15 functionals were combined and applied to the dissociation of $\mathrm{NH}_{3}$, which exhibits a conical intersection between the $\mathrm{S}_{1}$ and $\mathrm{S}_{0}$ states, and to the pyramidalization and twisting of ethylene. The DFT results show very close agreement with the PES calculated with CASPT2 and could provide a new approach to tackling difficult intersection points.

\subsubsection{Simplified Tamm-Dancoff approximation}

Grimme et al. have performed a wide-range of studies focusing on both implementation and development of TD-DFT methods as well as their subsequent application to organic systems. The derivations for simplified TDA- ${ }^{154}$ and full TD-DFT ${ }^{155}$ are presented, which allow for the computation of excited states for large molecules, i.e. up to $\sim 1000$ atoms; systems that still today present a significant challenge. The sTDA and sTD-DFT methods yield significant speed-ups compared to the full calculations, and the STDDFT provides the benefit of more reliable oscillator intensities, crucial for simulation of various spectra, compared to sTDA, and exhibits similar calculation errors to that of the full calculations. A subsequent study ${ }^{156}$ extends sTDA from just global hybrid exchange-correlation functionals (XCFs) to several range-separated hybrids, namely, the $\omega \mathrm{B} 97$ family of functionals, PBE0, B3LYP, CAM-B3LYP, and LC-BLYP. The study finds that CAMB3LYP and $\omega$ B97X-D3 perform best for most excitations, and that in general the RSH functionals perform better than global hybrids. As well as this, a study $^{157}$ applying the $\omega \mathrm{B} 97 \mathrm{X}$ and BHHLYP in conjunction with sTDDFT to simulate the electronic circular dichroism (ECD) spectra of [16] helicene, finds good agreement with experiment; ECD spectra represent a challenge for TD-DFT as the spectra are sensitive to oscillator intensities. 
A further extension of STDA is tight-binding based (sTDA-xTB), ${ }^{158}$ which is a semi-empirical approach to speeding up the generation of ground-state Kohn-Sham orbitals and eigenvalues, upon which the sTDA method can be applied for generation of excited states. The method is found to perform well for both low-energy valence and Rydberg states and outperforms other semi-empirical methods. The sTDA-xTB method was then applied to several biomolecular systems (thousands of atoms present) in order to simulate their UV-vis and CD spectra, finding excellent agreement between experimental and calculated spectra. ${ }^{159}$ sTD-DFT and sTDA has was also applied to the ECD spectra of several large, highly $\pi$-conjugated systems ${ }^{160}$; namely, $\mathrm{C}_{76}$ fullerene, [11] helicene, part of a chiral $(11,7)$ carbon nanotube, and a section of a long, $\alpha$-helical polypeptide chain. The study notes the differences between TDA-TD-DFT and full TD-DFT in terms of computing excited state properties, notably the difference that can arise when calculating the rotary strengths, a signed property on which simulation of ECD spectra depends. The study shows that, for $\mathrm{C}_{76}$ fullerene and [11] helicene, TDA (and sTDA) TD-DFT produce rotary strengths opposite in sign to that of a full TD-DFT (and sTD-DFT), resulting in incorrect spectra that would affect any attempt of assignment. In the case of [11]-helicene, the ECD spectrum is reasonably well simulated, however, a band of opposite sign to experiment is observed in the $250-280 \mathrm{~nm}$, demonstrating that the incorrect signs of rotary strengths from (s)TDA is not systematic and cannot simply be multiplied by -1 . (s)TD-DFT is found to give spectra in excellent agreement with experiment when combined with the $\omega \mathrm{B} 97 \mathrm{X}-\mathrm{D} 3$ functional.

Full TD-DFT has also been applied to the examination of 20 different 3,4,5-triaryl-1-R-1,2-diphosphole derivatives, in order to determine their applicability as optoelectronic materials. ${ }^{161}$ The study found that structural changes, for example, introduction of substituents to the para-positions of the aryl groups, or changing the substitution of the phosphorus atoms, can be used to tune the HOMO-LUMO gap of the molecules. TD-DFT and TDA-TD-DFT were also applied to the study of some fluorescent benzimidazolylquinoxaline derivatives, ${ }^{162}$ finding good agreement between experiment and calculations using the PBE0 and B3LYP functionals.

\subsection{Dye chemistry}

\subsubsection{Overview}

One of the most popular applications of TD-DFT is the study of dyes, particularly in the context of dye-sensitized solar cells. Jacquemin has applied 
TD-DFT to a large range of systems from both a benchmarking and exploratory perspective. ${ }^{2,47,163-183}$ Among other things, the performance of TD-DFT with respect to reproducing several excited state phenomena exhibited by several types of dye (i.e., anthraquinones, cyanines, coumarins, naphthalimides, BODIPY and similar dyes, photoacids and biodyes, and photoswitches) has been examined. ${ }^{179}$ The properties modelled included absorption and emission spectra and understanding their band shapes, charge-transfer characteristics for optimization in solar cells, and excited state proton transfers/photoacidity. TD-DFT was found to accurately reproduce vertical excitation energies using hybrid functionals, e.g., B3LYP and PBE0, with MAE of $0.10 \mathrm{eV}$. Multiple linear regression, with parameterized components from both PBE0 and B3LYP, was found to reduce the MAE to $0.08 \mathrm{eV}$, and reduced the number of erroneously large deviations. However, TD-DFT was found to severely overestimate the transition energies of cyanine and its derivatives; for example, B3LYP exhibits MAE of $0.31 \mathrm{eV}$ when applied to cyanine, and B3P86 exhibits a MAE of $0.25 \mathrm{eV}$ when applied to anthocyanine. With respect to exploring dye-sensitized solar cells, the same authors ${ }^{179}$ note the importance for including the surface in the time-dependent calculation, as the surface has a polarizing effect on the molecular electronic structure which will affect absorption behavior. Finally, the authors find that TD-DFT can also be applied to photoreactivity, i.e., excited state intermolecular proton transfer and photoswitching, which moves TD-DFT away from simply calculating intrinsic properties on equilibrium geometries.

A review focused on the application of quantum chemistry to cyanine excited states ${ }^{172}$ finds that TD-DFT can be used to find a specific cyanine transition; the transition will be functional independent and upshifted in energy when the Tamm-Dancoff approximation ${ }^{130}$ is employed, and the respective excited state geometries are well modelled. Single reference methods with included dynamic correlation are required for acceptable reproduction of the absolute transition energies, however. A review into the photodeactivation dynamics of phosphors ${ }^{178}$ highlights the non-systematic errors exhibited by TD-DFT when applied to systems with transition metals present. $0-0$ energies and vibrationally resolved spectra are also reviewed, ${ }^{2}$ generally finding that M06-2 $\times$, B3LYP, M06, and $\omega$ B97X-D give small maximum absolute errors for absorption spectra, which increase for emission spectra. LC-PBE was found to perform poorly for both sets of spectra.

Jacquemin et al. have also employed TD-DFT for a range of systems. The second-order NLO behavior of a $\mathrm{Pt}(\mathrm{II})$ complex $^{171}$ was reported 
and investigated experimentally and with TD-DFT; using PBE0 to model the absorption behavior and $\omega \mathrm{B} 97 \mathrm{X}-\mathrm{D}$ to compute the first hyperpolarizabilities. The computed absorption spectra are in good energetic agreement with the experimental spectra. Benelhadj and Jacquemin et al. ${ }^{176,184-186}$ have published studies into excited state intramolecular proton transfer processes in benzazole ${ }^{184}$ and benzoxazole ${ }^{176}$ derivatives, and the luminescent properties of borate complexes. ${ }^{185,186}$ The benzoxazole derivatives were found to exhibit fluorescence of white light, arising from dual fluorescence from enol and keto phototautomers. TD-DFT was employed to explore the excited state PES for excited state intramolecular proton transfer. ${ }^{165,174}$ This study was subsequently expanded to over 110 potential excited state intramolecular proton transfer dyes with a TD-DFT/ADC(2) hybrid approach. ${ }^{175}$ TD-DFT was used for vibrational and structural parameters, and ADC(2) was used for absolute and transition energies. This hybrid approach was found to give good agreement with experimental fluorescence spectra and, more importantly, consistently predicted whether fluorescence would arise from the keto or enol forms, or if dual fluorescence would dominate. Diketopyrrolopyrrole molecules ${ }^{170}$ were investigated for their solar cell sensitizing properties; specifically 4 different dyes were investigated. Experimentally, each of the dyes exhibit absorption at similar wavelengths, which is captured by TD-DFT. However, changing terminal thiophene groups for trisarylamine groups was found to enhance CT character in the excited states, a property beneficial for sensitizing dyes. The CT character was found to be well modelled by the CAM-B3LYP functional.

\subsubsection{Dye-sensitized solar cell applications}

Le Bahers et al. ${ }^{187}$ have discussed the proper practice for examining dyesensitized solar cells with quantum chemistry; summarizing the procedure as: (a) compute the structural and electronic properties of the dye-sensitized solar cell; (b) employ diagnostic tools to determine the accuracy and reliability of the calculations; (c) assess impact of dye adsorption; and (d) evaluate electron injection efficiency, incident photon-to-charge carrier efficiency (IPCE), and $\mathrm{J}_{\mathrm{SC}}$. Coupled with the PBE0 functional, three similar pyridinium derivatives were examined, finding good agreement with experimentally obtained results.

Mathew et al. ${ }^{188}$ report the success of two bis $\left(2^{\prime}, 4^{\prime}\right.$-bis(hexyloxy)- $\left[1,1^{\prime}-\right.$ biphenyl]-4-yl)amine-functionalised porphyrin-based dyes for solar cell sensitization, which exhibit power conversion efficiencies of up to $13 \%$. 
The two dyes are similar in that their donor groups (porphyrin core + amine groups) are identical but differ in that their acceptor groups are either a benzoic acid group (dye SM371), or a benzothiazole group (dye SM315); SM315 is found to be the more efficient of the two dyes. The M06 functional was employed in order to rationalize the observed differences in absorption behavior, finding that SM315 exhibited a greater amount of donor $\rightarrow$ acceptor CT character in the $\mathrm{S}_{1}$ state than SM371, as well as a series of bright absorption peaks extending to $\sim 400 \mathrm{~nm}$, which are used to explain the observed panchromatic absorbance of the dye and the resulting efficiency.

Liu et al. ${ }^{189}$ employed B3LYP and CAM-B3LYP to study the linear and non-linear optical properties of 6 derivatives of triphenylamine (-indandione) chromophores. Here, CAM-B3LYP was employed to optimize the ground state structures of the molecules as B3LYP is said to be less capable of reproducing the correct geometries or modelling the charge transfer taking place to give rise to the non-linear optical properties. However, it appears that B3LYP was nonetheless employed to simulate the absorption and emission spectra of the molecules, rather than CAMB3LYP. The authors do, however, find good agreement between the simulated and experimental spectra, and find that asymmetric modification of indandione groups results in a profound effect on the calculated first-order hyperpolarizabilities.

Linares-Flores et al. ${ }^{190}$ also used (CAM-)B3LYP to study the effect of different electron-donating substituents and anchors on the optical properties of porphyrin-based zinc phthalocyanine dyes. The ground state geometries were obtained using both functionals, with B3LYP giving a band gap in good agreement with experimental values, $3.2 \mathrm{eV}$ vs $3.4 \mathrm{eV}$, respectively; however, CAM-B3LYP gave an estimated band gap of approximately $6 \mathrm{eV}$, a somewhat spectacular failure. However, reasonable agreement is obtained for each functional between the calculated vertical excitation energy and oscillator strengths and experimental values obtained from similar compounds. The work was motivated by applications in dye-sensitized solar cells, and the study demonstrates that it is possible to use theory to optimize structural parameters so as to increase the efficiency of the electron injection process critical to photovoltaic action.

An earlier study by Zarate et al. ${ }^{191}$ also studied dye-sensitized solar cell models, employing both B3LYP with added dispersion corrections and CAM-B3LYP. Here, CAM-B3LYP was employed to calculate the vertical excitation energy, and a comparison to values as calculated by the 
$\omega$ B97X-D, BHHLYP, M06-2×, M11, HISSbPBE, HSE06, M06, PBE, and TPSSh functionals was performed. It was found that a smaller number of bridging units between the chromophore and the dye-sensitized surface gave rise to a larger absorption. Zarate et al. ${ }^{192}$ also studied the electron photoinjection mechanism and photovoltaic properties of $\mathrm{TiO}_{2}$-adsorbed donor-acceptor bridges with the B3LYP, CAM-B3LYP, PW91, PBE, and M06-L functionals. In total, 11 different donor motifs were compared to alizarin and napthalenediol, which can undergo both Type I and Type II photoinjection mechanism mechanisms, and it was found that different donor systems were capable of driving favorability for a photoinjection mechanism.

Li et al. ${ }^{193}$ used B3LYP (ground state geometries), CAM-B3LYP (absorption/emission), and M06-2 $\times$ (dye regeneration mechanism) to study various properties of three organic dyes, to determine their potential for application to dye-sensitized solar cells. The simulated absorption spectra are in good agreement with experiment, and charge density plots of the $\mathrm{S}_{0}$ and $S_{1}$ states show clear charge transfer from the donor to the acceptor regions of the molecule, suggesting CAM-B3LYP can reproduce these effects. In general, the study shows that CAM-B3LYP was capable of reproducing experimental observations, including which species had the higher molar absorption characteristics, and that substitution along the $\pi$-bridge had a significant impact on the absorption properties of the molecule.

Nachimuthu et al. ${ }^{194}$ studied the effects of anchoring groups on the optical and aggregation properties of a 4-methoxy- N-(4-methoxyphenyl)- $\mathrm{N}$ phenylbenzeneamine donor moiety coupled with several different acceptor and anchoring units. In particular, the role of the anchoring groups in dye aggregation was examined as extensive aggregation can result in a decrease in electron transport through a solar cell, and hence a decrease in its efficiency. The B3LYP, $\omega$ B97XD, BHHLYP, M06-2 $\times$, and CAM-B3LYP functionals were used in the excited state calculations, with the $\omega \mathrm{B} 97 \mathrm{XD}$ proving to be the most accurate. The study finds that dyes with a dithioic (CSSH) anchoring group exhibit the lowest energy transitions, and that the inclusion of a CN group into the anchoring moiety can also enhance absorption. Compared to the $\mathrm{COOH}$ anchoring group, molecular dynamics (MD) calculations found that dyes with CSSH favored aggregation to a greater extent, demonstrating that optimal choice of the anchoring group can be used to enhance favorable, and limit unfavorable, physical characteristics.

Baheti et al. ${ }^{195,196}$ used B3LYP to study the effect of functionalizing fluorene-based sensitizers for dye-sensitized solar cells. In their first study, 
one or two fluorenylidene moieties were incorporated into triarylamine donor units, coupled with cyanoacrylic acceptor units and (bi)thiophene conjugated bridges. All of the fluoro-containing dyes studied exhibit significant charge-transfer character arising from the HOMO-LUMO transition, and they also have red-shifted absorbance compared to the reference and dicyanovinyl containing dyes. As well as this, the fluoro-containing dyes exhibited higher molar extinction coefficients and HOMO-LUMO energy levels close to that of $\mathrm{TiO}_{2}$ that resulted in a higher photocurrent density than that arising from the dicyanovinyl species. In their second study the authors studied the effect of functionalizing rod-like and T-shaped dyes. They found that the rod-shaped dyes exhibit lower-energy $\mathrm{S}_{0} \rightarrow \mathrm{S}_{1}$ transitions compared to that of T-shaped dyes, and this arises from the extended delocalization found in the rod-like dyes. As well as this, it is found that (bi) thiophene linkers result in a red-shift in absorbance. Finally, while the rodlike have a lower-energy absorbance compared to the T-shaped dyes, the latter are in fact the more efficient sensitizers if they were to be used in a dye-sensitized solar cells.

Venkateswararao $^{197}$ used B3LYP and MPW1K to characterize the behavior of carbazole donor units with $\pi$-linkers. The calculations demonstrate significant charge-transfer character from the HOMO-LUMO transition at energies appropriate for electron injection to $\mathrm{TiO}_{2}$. Feng et al. ${ }^{198}$ use B3LYP and CAM-B3LYP in order to simulate the spectra of several molecules functionalized with an ullazine donor group, finding that the spectra demonstrate the applicability of ullazine to dye-sensitized solar cells.

Kulinich et al. ${ }^{199}$ used the B97D3, B3LYP, B3PW91, and $\omega$ B97X-D functionals to study the excited states of merocyanine. The study finds good agreement for all functionals between the calculated and experimental spectra, with the B97D3 functional performing best. The B97D3 functional suggests that the low energy, $\mathrm{S}_{0}$ to $\mathrm{S}_{1}$ transition is a $\pi \pi^{*}$ transition at $2.81 \mathrm{eV}$, in very close agreement with the experimental band centered at $2.8 \mathrm{eV}$. The $\mathrm{S}_{0}$ to $S_{2} / S_{3}$ excitations are found to be symmetry forbidden and therefore unresolvable in either experimental or simulated spectra. The B3LYP, B3PW91, and $\omega$ B97X-D functionals consistently overestimate the vertical excitation energy, with the $\omega \mathrm{B} 97 \mathrm{X}-\mathrm{D}$ energies being the least accurate.

Kowalska-Baron et al. $^{200}$ used the B3LYP and CAM-B3LYP functionals, as well as the $\omega \mathrm{B} 97 \mathrm{X}-\mathrm{D}$ functional, to study the distyrylnaphthalene chromophore. However, only the B3LYP functional was employed to study the excited state. Again, no description of the character of the $S_{1}$ state is included. The experimental absorption and fluorescence spectra are also 
broad and ill-defined, meaning it is unclear if the excited states are being correctly modelled. The chromophore also has an extensive $\pi$-system and the paper also mentions that charge transfer takes place in the $S_{1}$ state, for which TD-DFT, and B3LYP in particular, is known to fail. ${ }^{131-133}$ It is unclear, then, if the TD-DFT calculations are properly describing either the ground or excited state structures and energies of the chromophore.

In other studies, Lee et al. ${ }^{201}$ employed B3LYP to study the excited states of several fluorene containing iridium complexes. The complexes exhibit "deep-blue" phosphorescence, and significant metal-to-ligand chargetransfer character is found for the triplet excited states, suggesting significant spin orbit coupling between the excited states. Estrada et al. ${ }^{202}$ provide insight into the change in electronic structure of isoindigo upon functionalization. Different functionalization patterns are found to change not only the broadness of the absorption spectra but also the oscillator intensities. Thienyl groups were found to planarize the molecules. Lourenço Neto et al. ${ }^{203}$ employed B3LYP to simulate the absorption spectra of eight different phenolic acids, which were then be compared to the experimentally obtained spectra of four Brazilian fruits. From the calculations, the B3LYP spectra are in good agreement with the experimentally obtained absorption spectra, and the acidic makeup of the fruits can be elucidated.

\subsection{Excited state intramolecular proton transfer}

Excited state intramolecular proton transfer studies have also found TD-DFT a useful and often accurate method. Huang et al. ${ }^{204,205}$ employed TD-DFT to study the excited state intramolecular proton transfer mechanisms of 3-hydroxy-2-(thiophen-2-yl)chromen-4-one (3-HTC) and 2,5-bis(benzoxazol-2-yl)thiophene-3,4-diol with B3LYP. In their earlier study, 3-HTC is found to undergo excited state intramolecular proton transfer due to the strengthening of an internal $\mathrm{OH}$ hydrogen bond upon excitation. The $\mathrm{S}_{0}$ and $\mathrm{S}_{1} \mathrm{H}$-transfer PES were calculated and the favorability for excited state intramolecular proton transfer is clear. The simulated absorption and fluorescence spectra are in good agreement with the experimental spectra. Similarly, upon excitation an internal $\mathrm{NH}$ hydrogen present in 2,5-bis(benzoxazol-2-yl) thiophene-3,4-diol is strengthened, and the calculated PES show clear favorability for the excited state intramolecular proton transfer. However, with 2,5-bis(benzoxazol-2-yl) thiophene-3,4-diol, a double excited state intramolecular proton transfer process is in fact favored. 
As with 3-HTC, good agreement between simulated absorption/emission spectra is obtained. Zhu et al. ${ }^{206}$ studied the excited state intramolecular proton transfer of five molecules containing the 10-aminobenzo[h] quinoline chromophore group.

Liu et al. ${ }^{207}$ studied the excited state double proton transfer mechanism of 8-hydroxyquinoline (8HQ) using the B3LYP and CAM-B3LYP functionals. The 8HQ molecules were micro-solvated with either $\mathrm{NH}_{3}, \mathrm{H}_{2} \mathrm{O}$, or $\mathrm{AcOH}$. The HOMO-LUMO transitions for each species is of $\pi \pi^{*}$ character, and the strength of the $\mathrm{H}$-bond between the $8 \mathrm{HQ}$ and solvating species results in slightly different $\mathrm{H}$-transfer mechanisms. With $\mathrm{NH}_{3}$, the $\mathrm{OH}(8 \mathrm{HQ})$ $\mathrm{H}$-bond is found to be strongest and is therefore the first proton transfer to take place, followed by an $\mathrm{H}$-transfer from the $\mathrm{NH}_{4}^{+}$species back to the $8 \mathrm{HQ}$. The opposite is found to be true for the $8 \mathrm{HQ}-\mathrm{AcOH}$ species, and with $\mathrm{H}_{2} \mathrm{O}$, each of the two $\mathrm{H}$-bonds are found to be similar in strength, which leads to a concerted double proton transfer. Ground state analysis of the proton transfer for the $8 \mathrm{HQ}-\mathrm{NH}_{3}$ species shows a significant activation barrier, whereas TD-DFT results suggest a near-barrierless PES in the $S_{1}$ state.

Pina et al. ${ }^{208}$ studied the excited state intramolecular proton transfer of Indigo and a monohexyl-substituted derivative with the CAM-B3LYP functional. The study aims to provide evidence for whether the transfer is either a single or double excited state intramolecular proton transfer, and if the transfer involves rotation about the central carbon-carbon bond. It is found that only a singlet excited state intramolecular proton transfer takes place, and rotation is feasible but according to experimental ultrafast spectroscopy it is a non-competitive pathway.

The excited state intramolecular proton transfer of 1,5-dihydroxyquinone was studied by Zhou et al. ${ }^{209}$ with B3LYP. The simulated absorption and fluorescence spectra are found to agree well with experimental spectra, and the intermolecular $\mathrm{H}$-bonds are found to be strengthened upon excitation to the $S_{1}$ state. It is also found that a single excited state intramolecular proton transfer takes place on the $T_{1}$ surface. The $S_{0}$ to $S_{1} / S_{2}$ transitions are likely to be $\pi \pi^{*} / n \pi *$ transitions, respectively, based on the relative oscillator intensities of the two transitions ( 0.2866 vs 0 , respectively). The population of the $T_{1}$ state is attributed to internal conversion to the $S_{1}$ state followed by ISC to the $T_{1}$ state; however, no description of the character of the $T_{1}$ state is included, on which the efficiency of the ISC is dependent. It may be found that the higher $n \pi^{*}$ state may be involved in populating the triplet states, however this possibility is not explored. 
Li et al. ${ }^{210,211}$ used TD-B3LYP to explore excited state intramolecular proton transfer in 6-amino-2-(2'-hydroxyphenyl)-benzoxazole (6A-HBO) with and without an explicit methanol molecule present. The role of the methanol hydrogen bond does not appear to be significant; the uncomplexed 6A-HBO exhibits a H-transfer transition state energy that lies $9.6 \mathrm{kcal} \mathrm{mol}^{-1}$ above the ground state and a shallow $\mathrm{H}$-transferred minimum, and upon excitation the transition state decreases in energy to $8.7 \mathrm{kcal} \mathrm{mol}^{-1}$ and a slightly deeper $\mathrm{H}$-transferred minimum. Complexation to the $\mathrm{MeOH}$ molecule serves to stabilize the transition states for both the $\mathrm{S}_{0}$ and $\mathrm{S}_{1}$ state, to $6.4 \mathrm{kcal} \mathrm{mol}^{-1}$ and $5.2 \mathrm{kcal} \mathrm{mol}^{-1}$, respectively. Questions remain about the accuracy of B3LYP for predicting transition state energies, it would be interesting, therefore, to assess if other DFT functionals give similar potential surfaces. The authors also studied $6 \mathrm{~A}-\mathrm{HBO}$ and 5 -amino-2- $\left(2^{\prime}-\right.$ hydroxyphenyl)benzoxazole (5-HBO) excited state intramolecular proton transfer in the context of the role of the amino group, finding again that the $\mathrm{OH} \rightarrow \mathrm{N}$ hydrogen bond is significantly increased in strength in the $\mathrm{S}_{1}$, hence promoting excited state intramolecular proton transfer.

Zhang and $\mathrm{Liu}^{212}$ used TD-DFT to study the absorption behavior of 2,4,6-trisbenzothiazolylphenol, which can undergo excited state intramolecular proton transfer upon excitation the $S_{1}$ state. The authors note that design of molecules that undergo excited state intramolecular proton transfer can be difficult and here, TD-B3LYP calculations were used to show that the HOMO-LUMO gap can be red-shifted by increasing the extent of conjugation in the molecule. The calculated absorption (384 nm) and emission $(619 \mathrm{~nm})$ maxima were also found to be in good agreement with experimental values, at 376 and $570 \mathrm{~nm}$, respectively.

Zhao et al. studied the excited state intramolecular proton transfer mechanisms of 3-hydroxyisoquinoline (3HIQ), ${ }^{213}$ bis-2,5-(2-benzoxazolyl)hydroquinone (BBHQ) and its derivatives, ${ }^{214}$ and 1,8-dihydroxybenzo $[a, h]$ phenazine $(\mathrm{DHBP})^{215}$; each study used TD-B3LYP. In the case of $3 \mathrm{HIQ}$, three model proton transfers were considered; the first involved a single 3HIQ molecule along which the enol-keto tautomerization proceeds via a high barrier of approximately $37 \mathrm{kcal} \mathrm{mol}^{-1}$. The second and third transfer reactions involved (a) 3HIQ self-association in cyclohexane with a step-wise double proton transfer via a $3 \mathrm{HIQ}+\mathrm{H}^{+} / 3 \mathrm{HIQ}^{-}$chargeseparated intermediate, and (b) 3HIQ H-bonding to an acetic acid molecule that involves a simultaneous $3 \mathrm{HIQ} \rightarrow \mathrm{ACID} / \mathrm{ACID} \rightarrow 3 \mathrm{HIQ}$ double proton transfer to form the keto species. Mechanism (b) is found to be essentially barrierless. The studies of BBHQ and DHBP also employed a TD-DFT 
PES and demonstrated the feasibility for either single- or double-proton transfer mechanisms, as the respective hydrogen-bonds of interest are strengthened in the $S_{1}$ states. However, no work is done to definitively determine which mechanism actually takes place.

Liu et al. ${ }^{216}$ performed a similar study focusing on quinoline-pyrazole isomerization, which involves excited state intramolecular proton transfer. Two isomeric systems were considered, labelled QP-I and QP-II, as it is found experimentally that, upon photoexcitation, QP-I undergoes emission at a wavelength corresponding to the proton-transferred (PT) isomer; however, no such emission is observed for QP-II. The authors find that the PES for H-transfer in QP-II exhibits a lower barrier than that of QP-I on the $\mathrm{S}_{1}$ state, suggesting QP-II excited state intramolecular proton transfer is in fact more favorable than that of QP-I. The lack of emission is therefore attributed to the small energy gap $(\sim 0.29 \mathrm{eV})$ between the $\mathrm{S}_{1} / \mathrm{T}_{2}$ states found in PT-QP-II, which could result in ISC and therefore no fluorescence. The equivalent energy gap in PT-QP-I is found to be $\sim 0.77 \mathrm{eV}$, which results in the observed fluorescence.

\subsection{Photoinitiation}

Photoinitiation is the process by which involves a molecule that undergoes $\alpha$-decomposition (Type I) or a hydrogen atom abstraction (Type II) to generate either free radicals or ions upon irradiation with (usually UV) light. ${ }^{217}$ Fig. 3 shows the both types of photoinitiation, adapted from ref. ${ }^{217}$

Huix-Rotllant et al. ${ }^{108,218,219}$ have applied TD-DFT to retinal, acetophenone, and photoinitiation of nitroxide mediated polymerization (NMP). In the case of retinal, TD-DFT and TDA-TD-DFT with the B3LYP, CAM-B3LYP, BHHLYP, M06-2 $\times$, and mPW2PLYP functionals was compared to MRCISD + Q, SF-EOMCCSD $(\mathrm{dT})$, and SA-CAS. The study explores the ground and excited state PES, finding generally that the single-reference TD-DFT was unsuitable, and was incapable of describing the diradical and CT intersection geometries. As well as this, TD-DFT was found to produce "kinks" in the PES, artefacts that were not present in higher-level calculations. Out of all the functionals tested, the double hybrid mPW2PLYP functional performed the best. mPW2PLYP was also found to perform the best in describing the three-state crossing between the ${ }^{1}\left(n \pi^{*}\right),{ }^{3}\left(n \pi^{*}\right)$, and ${ }^{3}\left(\pi \pi^{*}\right)$ states, in particular when combined with TDA-TD-DFT. The double hybrid functional was the only functional to predict a three-state crossing and produced energies and geometric 

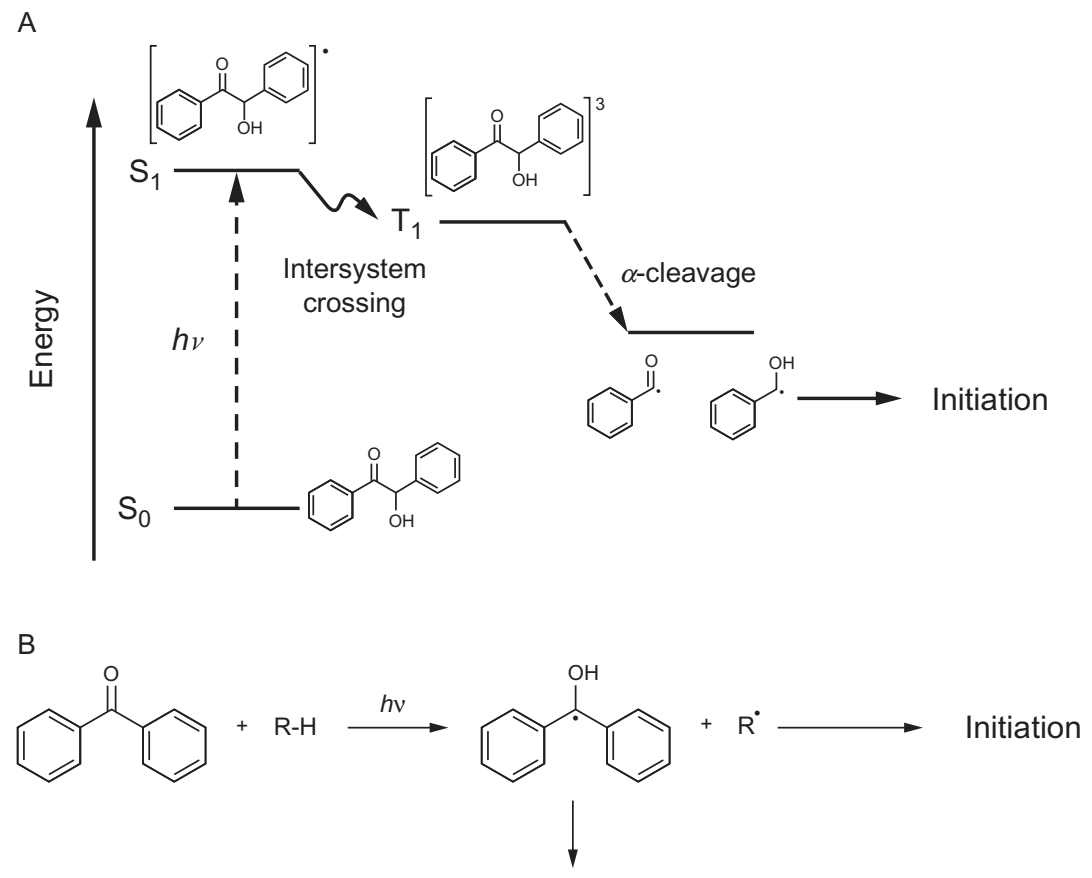

Side reactions

Fig. 3 Example mechanism for Type I (A) abd Type II (B) photoinitiation.

parameters in closest agreement with multireference calculations. The increase in accuracy of mPW2PLYP is attributed to the larger inclusion of double-excitations in the excited states arising from the MP2 component of the calculation. Full TD-DFT, and CAM-B3LYP in particular, was found to perform badly when describing the triplet surfaces, and TDATD-DFT is found to correct these issues.

For the NMP study, several different isomers of an NMP iniferter (An iniferter is an initiator for radical polymerizations.), which combine a chromophoric acetophenone moiety with an alkoxyamine group that will undergo cleavage to initiate the reaction, were studied using TD-B3LYP. The calculations suggest that the photochemical processes of the iniferters are defined by singlet-triplet crossing, followed by excitation delocalization across the alkoxyamine moiety, which results in bond homolysis. It was also found that $\mathrm{C}-\mathrm{N}$ and $\mathrm{O}-\mathrm{C}$ dissociations can compete, which could account for low initiation efficiencies. However, in their acetophenone study Huix-Rottlant et al. ${ }^{218}$ recommend the use of mPW2PLYP/TDA for exploring the excited states of acetophenone-based chromophores, 
not B3LYP/TD-DFT which is found to perform poorly. As well as this, B3LYP is known to fail for describing radical bond dissociations, ${ }^{220}$ so the competition between the different $\sigma$-cleavage pathways remains unclear.

Frick et al. ${ }^{221}$ explored the relationship between the structure and reactivity of benzoin-based radical photoinitiators, using a combination of pulsed-laser polymerization with subsequent electrospray-ionization mass spectrometry (PLP-ESI-MS), femtosecond transient absorption (fs-TA) spectroscopy, and ab initio calculations. TD-B3LYP was employed to compute the excited state properties, finding generally that there was no significant relationship existed between the brightness of an excited state and the subsequent dissociation reaction. In fact, dark $n \pi *$ states generally dominate the $S_{1}$ state, and it is the population of these states that most efficiently leads to dissociation. The study also finds that if too many singlet/triplet states lie below the likely most reactive states, photoinitiation becomes less favorable due to increased competition with internal conversion decay pathways.

Xiao et al. ${ }^{11,222,223}$ employed TD-DFT to study the absorption characteristics and photoinitiation mechanism of anthraquinone derivatives. The studies highlight the impact of different substitution patterns can have on altering the ordering of $n \pi *$ and $\pi \pi^{*}$ transitions, also finding that the anthraquinone molecules undergo excited state redox processes when co-initiating molecules are present.

\subsection{Other photochemical reactions}

A range of other photochemical triggered reactions are possible, and below is just a few of the processes studied via TD-DFT. Luo et al. ${ }^{224}$ employed ultrafast spectroscopic techniques and TD-M06-2 $\times$ to investigate the efficacy of sinapoyl malate and sinapic acid for natural UV-protection. In this case, TD-DFT is found to be able to qualitatively reproduce the $\mathrm{S}_{0 / 1} \mathrm{PES}$, finding that the cis-trans isomerization is barrierless for both systems on the $\mathrm{S}_{1}$ state, and leads back to the ground state. However, it is observed that the cis-isomer of sinapic acid is less able to absorb UV light than its transcounterpart; in contrast, sinapoyl malate exhibits no significant difference in the absorption characteristics of either cis/trans isomer and continues to absorb UV light efficiently. This is used to reconcile why sinapoyl malate is prevalent in nature rather than sinapic acid.

Mazzone et al. ${ }^{225}$ used several functionals, M06-2 $\times$, M06, PBE0, and B3LYP, to examine the electronic properties and spin-orbit couplings of two thienopyrrole BODIPY (BODIPY =a boron-dipyrromethene). 
All four functionals gave consistent structural parameters and $S_{1} / T_{1}$ energy gaps, and M06 and B3LYP gave $S_{0}$ to $S_{1}$ transition energies closest to the experimental values. The spin orbit coupling between the $S_{1}$ and $T_{1}$ states was found to be higher than Foscan ${ }^{\circledR}$ (Temoporfin photosensitizer) taken as a reference molecule, and the BODIPY derivatives are therefore feasible photosensitizers for singlet oxygen generation.

Liu et al. ${ }^{226}$ designed a cross-coupling reaction between thiol and aryl halides that is promoted by visible light, and results in a highly synthetically useful carbon-sulfur bond. TD-DFT was applied in order to gain insight into the process taking place upon excitation, using the CAM-B3LYP functional. The calculations suggest that, upon excitation, a preformed negatively charged electron donor-acceptor (EDA) complex undergoes CT to form a negatively charged species now on the acceptor molecule, and a biradical across the complex. These biradicals are localized on the carbon and sulfur atoms that then undergo coupling to form the product.

Kozlowski et al. ${ }^{227}$ give a perspective on the photochemistry of cobalamins; in particular, methylcobalamin (MeCbl), adenocobalamin (AdoCbl), cyanocobalamin $(\mathrm{CNCbl})$ a.k.a. vitamin- $\mathrm{B}_{12}$, and hydroxocobalamin (HOCbl). These molecules have significant biological relevance, for example, some enzymes are found to be dependent on AdoCbl in radical-mediated rearrangement reactions; the cobalamins also possess a "unique $\sigma \mathrm{Co}-\mathrm{C}$ bond". The rich chemistry of the cobalamins also involves complex excited state properties that are found to be dependent not only on the relative substitutions of various chemical groups, but also the local environment. In particular, cobalamins are found to undergo bond photolysis, resulting in a $\mathrm{Co}(\mathrm{III}) \rightarrow \mathrm{Co}(\mathrm{II})$ oxidation and production of alkyl radicals, and detailed understanding of the photolysis mechanism is therefore sought. Due to the size of the systems, TD-DFT was employed rather than ab initio methods, along with a significantly truncated model that captures the cobalamin Co core and leaves out any functional groups extending beyond the pyrrolic halo. B3LYP, and dispersion corrected B97-D and BP86 functionals were used, finding that, for MeCbl, B3LYP incorrectly predicted a $\mathrm{S}_{1}$ state dominated by $\pi \pi^{*}$ character, whereas BP86 correctly (according to ab initio and spectroscopic results) predicts predominantly metal to ligand charge transfer character. The results concentrate on the singlet excited states of the four cobalamin complexes, finding that cobalamin low-lying excited states can be dominated by $\pi \pi^{*}, \sigma \pi^{*}, \pi d$, and $d \pi^{*}$ characters, which give rise to complicated dissociation pathways from which reactivity can be difficult to elucidate. As well as this, the authors find that TD-DFT is insufficient to 
explain the experimentally observed wavelength independence of AdoCbl with respect to photolysis, as the calculations draw similarities between AdoCbl and $\mathrm{MeCbl}$ that do not translate to experimental similarities. The involvement of triplet excited states is also fielded as a potential avenue for further study and could provide further insight into currently unexplained phenomena.

Ding and $\mathrm{Liu}^{228}$ used TD-DFT and SF-TD-DFT to study the bioluminescence mechanism of luciferin in Watasenia scintillans. The bioluminescence of this structure is important it is a derivative of a core skeleton structure that is found in wide range of bioluminescent organisms. CAMB3LYP was applied to TD-DFT calculations, from which a range of ground- and excited-state PES were calculated, to either support or refute the proposed bioluminescence mechanism. ${ }^{229}$ As well as this, BHHLYP was used in conjunction with SF-TD-DFT to perform non-adiabatic dynamics simulations with on-the-fly trajectory surface hopping (THP) to model the chemiluminescent decomposition reaction. SF-TD-DFT was chosen as it is more capable of coping with regions of electronic degeneracy. These two methods were found produce qualitatively correct surfaces and results, from which the previously proposed mechanism is strongly supported.

\subsection{UV-Vis spectra and excitation energies}

Ashwood et al. ${ }^{230}$ studied the excited state dynamics of 6-thioguanine (6tGua) and its N9-glycosilated derivative (6tGuo) with ultrafast spectroscopy, and TD-DFT was used to provide theoretical insight; the PBE0 functional was used. The calculations find low-lying triplet states that are close in energy to the $S_{1}$ and $S_{2}$ states. The calculations and dynamics experiments appear to disagree, as the difference in the vertical excitation energy of 6tGua and 6tGuo are small, however, upon the excitation, the triplet states of the 6tGuo are populated nearly twice as quickly as the 6tGua molecule. This is attributed to an increase in vibronic coupling between various singlet and triplet states due to the 6tGuo molecule being significantly larger.

Simone et al. $^{231}$ studied the effect of substituting hydrogen atoms for either bromine or iodine atoms at various positions of aza-BODIPY. Aza-BODIPY has potential for therapeutic use as a photosensitizer for generation of singlet oxygen, which is cytotoxic and can be used to kill targeted cells. For a photosensitizer to be efficient, the triplet states of the molecule need to be effectively and rapidly populated after excitation. The M06 functional was used to calculate the ground state and absorption properties of the 
substituted derivates, finding that neither bromine nor iodine had a significant impact on the absorption properties. TD-DFT also predicted that for all compounds investigated, the lowest energy transition is the $\pi \pi^{*}$ transition, and that the low-lying triplet states are of $n \pi^{*}$ character, and therefore populating these states, according to the El-Sayed rules, ${ }^{232}$ should be reasonably favorable.

Boron dipyridylmethene (DIPYR) dyes, which are pyridine based BOPDIY derivatives, were studied by Golden et al. ${ }^{233}$ TD-DFT and XMCQDPT2 calculations were performed on boron dipyridylmethene, boron diquinolylmethene, and boron diisoquinolylmethene. B3LYP was employed, and the calculated vertical excitation energies were found to be systematically overestimated and were subsequently scaled by subtracting $0.44 \mathrm{eV}$ from all calculated values; doing so resulted in good agreement with experimental values for the three molecules. The lack of fluorescence exhibited by DIPYR is attributed to the near degeneracy between the $\mathrm{S}_{1}$ and $T_{2}$ states which leads to efficient ISC between the two. The authors note that manipulation of the relative energies of these two states, e.g. by benzannulation, can lead to an increase in fluorescence behavior.

Lorenz et al. ${ }^{234}$ studied the electronic structure of poly( $p$-phenylene iminoborane) with TD-B3LYP. This was done to determine the extent of the $\pi$-conjugation across the polymer backbone; the TD-DFT calculations were performed to find the HOMO-LUMO transition, which was found to be a $\pi \pi^{*}$ transition, providing evidence that there is such a $\pi$-system. Three different chain lengths were studied, and it was found that conjugation extends with chain length.

Margar et al. ${ }^{235}$ studied difluoroboron-curcumin complexes, another highly $\pi$-conjugated system, in which the hydroxy-groups were replaced with methoxy and tosylate groups. Absorption and emission properties were calculated with B3LYP and are in good agreement with experimental values, and the first-order hyperpolarizability was calculated, suggesting that the molecules could exhibit significant non-linear optical properties. Kamada et al. ${ }^{177}$ also studied difluoroboron curcuminoid molecules with TD-B3LYP, using it to calculate the one- and two-photon absorption properties. In agreement with Margar et al., the fluorophores are shown to exhibit significant non-linear optical properties, and B3LYP calculations are in good agreement with the experimental absorption spectra, if somewhat blue-shifted.

Cabrera-González et al. ${ }^{236}$ studied a set of carborane clusters for their photophysical properties, finding experimentally that ortho- vs meta- bonding to the stilbene groups does not affect the absorption profile of the 
different molecules. TD-B3LYP found that for all systems the $\mathrm{S}_{1}$ state was dominated by a HOMO-LUMO transition localized to the stilbene moieties, leading to absorption behavior independent to the bonding patterns of the stilbene units. This is found to be in direct contrast to previously reported carborane diads, ${ }^{237,238}$ which find that the $\mathrm{S}_{1}$ states are dominated by CT character and are therefore significantly affected by structural parameters.

Momeni and Brown ${ }^{239}$ note that BODIPY and aza-BODIPY are challenging systems for TD-DFT to model accurately, and therefore benchmark the performance of a range of functionals (B3LYP, PBE, BLYP, PBE0, LC-BLYP, LC-PBE, CAM-B3LYP, $\omega$ B97X-D, and LC- $\omega$ PBE) against $\mathrm{ab}$ initio methods (TD-HF, CIS, CIS(D), EOM-CCSD, SAC-CI, LCC2, CCS, CC2, LR-CCSD, CCSDR(T), CCSDR(3), CASSCF, and CASPT2), all with the cc-pVTZ basis set. The correlated methods provide the authors with the means to analyze the different contributions to the excited state wavefunctions, for example dynamic correlation arising from the coupled-cluster methods, or double excitations that can appear in CASSCF calculations. The study finds that TD-DFT generally overestimates the vertical excitation energy by $>0.3 \mathrm{eV}$, and that within the test-set of functionals used, the range-separated functionals generally performed the best. Although the ab initio methods are computationally expensive, the authors find that SAC-CI and LCC2 with reasonably sized basis sets (cc-pVDZ/cc-pVTZ) provide greater accuracy than TD-DFT, while still being applicable to medium-sized systems.

Fujikawa et al. ${ }^{240}$ were able to rationalise the UV-vis absorption behavior of two isomers, twisted- and meso-, of their synthesized double helicene molecule. The meso-isomer was found to exhibit a similar spectrum to that of the twisted-isomer, albeit shifted to higher energies. This was found, via TD-B3LYP, to arise from an increase in contribution from the HOMO-2 orbital to the $\mathrm{S}_{1}$ state for the meso-isomer, resulting in a higher energy excitation compared to that of the twisted-isomer. This is a neat example of how TD-DFT can be used to separate the behavior of two isomers.

Maier et al. report the seminumerical implementation ${ }^{241}$ and performance $^{242}$ of TD-DFT for global and local hybrid XCF functionals. The semi-numerical implantation is found to scale favourably compared to analytical implementations available for global hybrids. The performance of several popular XCFs namely SVWN, BLYP, PBE, TPSS, TPSSh, PBE0, B3LYP, BMK, BHLYP, M06-2X, CAM-B3LYP, LC- $\omega$ PBE, and $\omega \mathrm{B} 97 \mathrm{X}-\mathrm{D}$, is compared to that of the following local hybrid $(L h)$ functionals; Lh-SVWN, Lh-SsifPW92, and Lh-SsirPW92. The local hybrid functionals are found to perform well when calculating the singlet and triplet 
excitation energies of the Thiel benchmark set of molecules, ${ }^{243,244}$ and provide a good balance between accurate energies for valence, core, and Rydberg states. However, the local hybrids were found to perform poorly for charge-transfer excitations.

Zhekova et al. ${ }^{245}$ benchmark the performance of adiabatic TD-DFT (ATD-DFT) with and without the Tamm-Dancoff approximation. The performance of ATD-DFT is compared to that of CC2 and a $\triangle$ SCF procedure for energies corresponding to the first $\pi \pi^{*}$ transition in a number of cyanine molecules. The ATD-DFT excitation energies were found to be reasonably functional independent, however, this still corresponded to significant singlet and triplet energy over- and underestimation, respectively. The $\Delta$ SCF procedure was found to exhibit significant functional dependence, with the authors finding that the BHLYP functional with the proportion of exact HF exchange set at $50 \%$ gave the excitation energies closest to ab initio results.

Cao et al. ${ }^{246}$ studied the effect of antiaromatic bispentalene derivatives fused attached to either a benzene or naphthalene core. The focus of the paper is generally toward the experimental work carried out, showing with NMR that, although the molecules of interest fit the classical rule of aromaticity (i.e., $[4 n+1] \pi$ electrons), the pentalene protons experience a significant upfield shift like that of antiaromatic compounds. As well as this, ring currents around the rings were measured using nucleus-independent chemical shift (NICS)-XY-scans, which demonstrated a global paratropic ring current (consistent with antiaromaticity) and a local diatropic current around the benzene core. The result of these opposing ring currents is present in the UV-vis spectra which show, for all of the systems studied, broad, low-energy absorption characteristics. Here, TD-CAM-B3LYP was used to characterize these broad peaks finding, compared to fully aromatic molecules with the same number of fused rings, significantly lower HOMOLUMO energy gaps. Particularly impressive is the significant absorption taking place at $>650 \mathrm{~nm}$.

Etienne et al. ${ }^{247}$ developed a method of quantifying the extent of chargetransfer that takes place upon excitation, essentially by determining the extent of the overlap between the ground- and excited-state electron densities. The index, $\phi_{S}$, is found to be relatively insensitive to the type of orbital employed, e.g. natural transition orbitals (NTO) vs. Kohn-Sham, is computationally cheap to calculate. The index can also be employed to assess the failing of XCF functionals that are not range-corrected, so as to determine the extent of any potential XCF failing. 
Ji et al. ${ }^{248}$ synthesized several boron-containing oligothiophene chromophores, and employed TD-B3LYP in order to examine both the oneand two-photon absorption behavior of the molecules. The simulated one-photon absorption spectra are in good agreement with the experimental data, and the calculated TPA spectra are found to be systematically overestimated by B3LYP. However, the calculated TPA trends between each of the molecules studied were found to be reproduced qualitatively. In another interesting study Arulmozhiraja et al. ${ }^{249}$ used TD-DFT and SAC-CI to explain the strong UV-absorbance of Arsenicin A, a compound with no formal $\pi$ bonds. It was shown that the chromophore facilitated by through-space and through-bond interactions between the lone pairs on the arsenic and oxygen atoms and the $\sigma$-bonding framework of the molecule. These destabilize occupied and stabilize unoccupied molecular orbitals thus favoring the transitions.

\subsection{Thermally activated delayed fluorescence (TADF)}

Samanta et al. ${ }^{250}$ applied TD-DFT to the up-conversion intersystem crossing (UISC) of 11 different organic emitting molecules that undergo thermally activated delayed fluorescence (TADF). B3LYP was used to optimize the ground state structures, and CAM-B3LYP was used to optimize the excited state geometries. $\mathrm{S}_{1}$ and $\mathrm{T}_{2}$ states were optimized with TD-DFT, and the $T_{1}$ state was optimized with unrestricted DFT; the excitation energies, however, were calculated with a tuned LC- $\omega$ PBE functional. The spin-orbit couplings (SOC) between the singlet and triplet states of interest were also calculated, and it is well established that the smaller the energy difference between singlet and triplet states $\left(\Delta E_{T S}\right)$, the larger the SOC, and ultimately the faster the rate of UISC. The study finds that $\Delta E_{T S}$ cannot be reduced by simply extending the spatial separation between the donor-acceptor regions within the molecule; instead, it is found that increasing the $\mathrm{CT}$ character of the $\mathrm{T}_{1}$ results in a concomitant decrease in $\Delta E_{T S}$. It is also found that, if possible, the symmetry of the singlet and triplet states needs to be different, which according to El Sayed's rules results in an increase in SOC.

Cai et al. ${ }^{251}$ employed TD-DFT with the BMK functional to tune TADF of organic light emitting diodes (OLEDs). Four rate parameters are identified as important for determining the crucial excited state lifetime of TADF $\left(\tau_{\mathrm{TADF}}\right)$; the rate of fluorescence $\left(\mathrm{k}_{\mathrm{F}}\right)$, the rate of intersystem crossing (ISC, $\mathrm{k}_{\mathrm{ISC}}$ ), the rate of reverse ISC (RISC, $\mathrm{k}_{\mathrm{RISC}}$ ), and the rate of 
internal conversion (IC, $\left.\mathrm{k}_{\mathrm{IC}}\right)$. $\tau_{\mathrm{TADF}}$ is required to be short for OLEDs to be at their most efficient, and it is found that increasing $\mathrm{k}_{\mathrm{RISC}}$ has the most profound effect in shortening $\tau_{\mathrm{TADF}}$, even when large values of $\mathrm{k}_{\mathrm{ISC}}$ and small values of $\mathrm{k}_{\mathrm{F}}$ are observed. It was also found that tuning the dihedral angles of the molecule can decrease $\Delta \mathrm{E}_{\mathrm{ST}}$; these factors are to be considered when designing OLED candidate systems.

Gómez-Bombarelli et al. $^{252}$ applied a high-throughput screening approach to target novel TADF OLED candidates. Ultimately TD-B3LYP was employed to quantify the excited state properties of the molecules of interest, finding that it performed well for calculating vertical absorption. Improvement in the calculated values were not obtained though usage of M06- $2 \times$, BHHLYP, LC- $\omega$ PBE0, CAM-B3LYP, or $\omega$ B97X-D functionals, nor through employing the PCM implicit solvent model. It was found that TD-B3LYP was incapable of accurately reproducing $\mathrm{S}_{1}$ PES as it overestimated the extent of the CT character of the excited state, and UDFT was therefore employed to optimize the $\mathrm{T}_{1}$ state. It is unclear if basis set choice was also considered for the calculations, as the $6-31 \mathrm{G}(\mathrm{d})$ basis set used is lacking the diffuse functions generally required to model the more diffuse excited state electron densities.

Valchanov et al. ${ }^{253}$ also examined TADF of a series of donor-bridgeacceptor organic chromophores using the B3LYP and PBE0 functionals. The absorption and emission spectra were simulated, and singlet-singlet and singlet-triplet energy gaps were evaluated in order to determine the likelihood of (R)ISC processes. The authors find that the optical properties can be significantly altered by varying any one of the following physical parameters; the spacer fragment used, the binding positions to it, and the structure/ elemental makeup of the donor and acceptor units. Chen et al. ${ }^{254}$ examined TADF from a more fundamental perspective; that is, exploring the role of nonadiabaticity in the observed RISC mechanisms. TD-BMK is employed to explore some of the potential energy surfaces of some donor-acceptor molecules.

Zhang et al. ${ }^{255}$ applied TD-DFT as a means to design anthraquinonebased TADF emitters, based on the requirement for a system that exhibits large fluorescence rates $\left(k_{F}\right)$ and small singlet-triplet energy gaps $\left(\Delta \mathrm{E}_{\mathrm{S}-\mathrm{T}}\right)$. In order to achieve these desirable qualities, the authors paired anthraquinone acceptor units with diphenylamine (DPA), bis(4-biphenyl)amine (BBPA), 3,6-di-tert-butylcarbazole (DTC), and 9,9-dimethyl- 9,10dihydroacridine (DMAC) as the donor units, using phenyl rings as the bridging units. The authors found that increasing the distance between the 
acceptor/donors increased the rate of fluorescence without increasing the $\Delta \mathrm{E}_{\mathrm{S}-\mathrm{T}}$, as is desirable. However, geometry optimizations of the molecules on the $S_{1}$ excited state found significant out-of-plane relaxation resulting in a decrease in $\Delta \mathrm{E}_{\mathrm{S}-\mathrm{T}}$ with a lower $k_{F}$ and an increase in radiationless internal processes. Predictions made with TD-DFT with respect to emission peaks and photoluminescence quantum yield $(\Phi)$ were found to be in good agreement with experimental observations, despite the fact the B3LYP functional was employed for systems that exhibit significant charge-transfer.

Walter et al. ${ }^{256}$ investigated the performance of TD-DFT and the semiempirical OM2 ${ }^{257}$ method against spin-component scaled (SCS-) CC2 and SCS-ADC(2) by application to perylene and perylene bisimide (PBI) dimer aggregates. The functionals used were CAM-B3LYP, $\omega$ B97X-D, $\omega$ B97X, LC- $\omega \mathrm{PBE}$, and LC-BLYP. The dimer aggregates are $\pi$-electron rich systems, and a significant component of the stabilizing energy holding the systems together is likely to arise from dispersion forces, however, no dispersion correction to any of the functionals was introduced so this effect has not been studied. The study finds that the CAM-B3LYP and $\omega$ B97X-D functionals give the best results for the perylene dimer, and for PBI the predicted ordering of the excited states is found to be incorrect when calculated with CAM-B3LYP. The $\omega$ B97X-D functional results were found to be extremely sensitive to the monomer geometries of PBI, and this was attributed to near-degeneracies in the low-lying excited states. The long-range corrected functionals were found to give qualitatively correct results but overestimated the CT state energies. A combination of $\omega \mathrm{B} 97 \mathrm{X}-\mathrm{D}$ monomer geometry and $\omega \mathrm{B} 97 \mathrm{X}$ transition energies was found to provide an accurate approach, and it was also found that tuning the $\omega$ parameter of $\omega \mathrm{B} 97 \mathrm{X}-\mathrm{D}$ to $\omega=0.25$ yielded results similar to the ab initio methods. However, the authors note that this approach needs to be further investigated. The OM2 method was found to give poor results.

Huang et al. ${ }^{258}$ examine the HF exact-exchange dependence with respect to the calculation of charge-transfer states in organic fluorophores that could be employed in TADF. The authors highlight the importance for the accurate prediction of singlet- and triplet-charge-transfer adiabatic energies for assessment of a molecules potential and employ a range of $\mathrm{XCFs}$ that incorporate different percentages of HF exchange, the most extreme examples being BLYP (0\% HF) to M06-HF (100\% HF). All the calculations point to a significant $\% \mathrm{HF}$ dependency when describing CT character. 


\subsection{Fluorescence and quenching}

Qian et al. ${ }^{259}$ used TD-DFT to gain insight into the suppression of Kasha's rule found in the fluorescent behavior of 8 derivatives of the BODIHY chromophore. The PBE functional was chosen, and its suitability was determined by comparison of calculated low energy bands against experimental absorption spectra, finding the predicted absorption maxima are within approximately $5 \mathrm{~nm}$ of the experimental values, and the predicted intensities are within approximately $10 \%$. The PBE functional also correctly predicts the observed blue-shift in absorption upon addition of ortho-methyl groups and the observed red-shift upon addition of para-electron withdrawing groups. TD-DFT calculations also find that the $\mathrm{S}_{1}$ state is not the first observable transition in the experimental spectra, instead the absorption maxima correspond to the $S_{0}$ to $S_{2}$ transition. It is also found that for the highly fluorescent molecules, the $\mathrm{S}_{2}$ state is significantly higher in energy than the $S_{1}$ state, and the less fluorescent molecules have a smaller $S_{1} / S_{2}$ energy gap. The difference in the energy gaps is inversely correlated to the efficiency of the $S_{2} / S_{1}$ internal conversion, the smaller the gap, the greater the extent of internal conversion. As the $S_{1}$ state is dark, this increase in population will result in less fluorescence, as observed.

Escudero $^{260}$ reviewed the oft-quoted mechanism for fluorescence quenching in donor-acceptor molecules, photoinduced electron transfer (PET). Escudero argues that PET is overused as a mechanism for fluorescence quenching in fluorescent sensors/switches and employs TD-DFT and multireference calculations to provide evidence for this. Instead of PET, Escudero finds that enhancement of "dark" excited states, e.g. $n \pi *$ states, are the most likely explanation, as they provide nonradiative pathways not previously accessible in the "ON" state of the sensor/switch. TD-PBE0 was found to be capable of reproducing adiabatic PES of the lowest-lying excited states and finds that multireference (CASPT2//CASSCF) calculations are required for proper treatment of any higher states that may be relevant.

Shepard et al. ${ }^{261}$ used increasing halogen substitution to form high-spin iron(II)-polypyridines complexes, in order to extend the excited-state lifetime of the metal-to-ligand charge-transfer (MLCT) excited states. The study employs the APFD hybrid density functional, and the TD-DFT calculations show that the low-lying states remain predominantly metalto-ligand, i.e. metal d-orbitals to ligand $\pi^{*}$-orbitals, in nature. It is also found that the increased number of chlorine atoms does indeed increase the MLCT lifetime, however, this evidence is obtained experimentally rather than from the TD-DFT calculations. 
Nobusue et al. $^{262}$ report the synthesis and physical properties of tetracyclopenta[def,jkl,pqr,vwx]tetraphenylene (TCPTP), which is found to potentially exhibit tetraradicaloid nature. Here, a combination of CASSCF and TD-DFT, using either BHHLYP or LC-BLYP, were used to study the structure and occupation of the LUMO and LUMO+1 of the species. Imposing $\mathrm{D}_{2 \mathrm{~h}}$ symmetry on the structures results in the lowest energy geometry with a relatively small diradical nature, whereas $\mathrm{D}_{4 \mathrm{~h}}$ symmetry resulted in a higher energy structure with a LUMO and LUMO +1 population (1.000 and 0.166 , respectively) indicative of tetraradicaloid character. TD-B3LYP was also employed, showing that it was possible to decrease the energy of the HOMO-LUMO transition of TCPTP by substituting extended conjugation onto the ring. $\mathrm{Hu}$ et al. ${ }^{263}$ studied the tetraradicaloid nature of $\mathrm{p}$-quinodimethane ( $\mathrm{p}-\mathrm{QDM}$ ) units fused to either a naphthalene or a benzene ring. DFT and TD-DFT calculations were used to study the UV-vis absorption of the molecules as well as study the orbital occupancies that would suggest polyradicaloid character of the species; in this case, the tetraradicaloid character of the molecules was found to be very small. However, different fusion modes of the p-QDM to the benzene/ naphthalene moieties had a pronounced effect on diradical characteristics.

Lou et al. ${ }^{264}$ note the advantages of using TD-DFT when exploring the photochemistry of redox-responsive fluorescent probes for use in biological systems. In particular, these complicated systems can give rise to experimental data that can be difficult to rationalize; however, such experimental results can be used in conjunction with TD-DFT calculations to determine the photophysical processes taking place, and therefore can well describe the biological system of interest. As well as this, the biological probes are generally very large, and can incorporate heavy metals, for example, selenium, and can therefore only feasibly be tackled with TD-DFT.

Daday et al. ${ }^{265}$ used a range of excited state methods, namely, TD-DFT (CAM-B3LYP, LC-BLYP), CASPT2, NEVPT2, and QMC, with lower level theories, i.e. static point charges (QM/MM), DFT embedding (QM/DFT), and classical polarizable embedding through induced dipoles (QM/MMpol), in order to determine which combinations of theory were able to fully capture the complex photophysical behavior of the green fluorescent protein (GFP), which is known to be dependent on its environment. The authors study the GFP in both its neutral and anionic form, finding better agreement for the anionic form of the GFP compared to the neutral species, which is found to exhibit greater sensitivity to the structure of the environment. This sensitivity is demonstrated by the spread of 
excitation energies found for several different "frames" in the MD simulations. It is also found that the QM/MM approach to the environment results in a systematic blue-shift in excitation energies, and the QM/DFT approach is also unable to provide results in good agreement with experiment. The QM/MMpol approach, specifically the linear-response (polLR) approach, results in a chromophore-protein resonance that leads to a red-shift in excitation energy and subsequently more accurate excitation energies. The TD-DFT energies were found to be blue-shifted relative to that of CASPT2, although both sets of energies followed a similar trend with respect to the lower-level approach to environment.

Wu et al. ${ }^{266}$ study the effect of aggregation on the absorption/emission characteristics of three highly conjugated systems; 9,10-distyrylanthracene (DSA), 2,3-dicyano-5,6-diphenylpyrazine (DCDPP), and cis,cis-1,2,3,4tetraphenyl- 1,3-butadiene (TPBD). Generally, it is found that upon aggregation optical emission spectra will be red-shifted relative to the solvent-phase species; however, the emission spectra of the three molecules investigated in this study are found to undergo a blue-shift upon aggregation. The authors employ a PCM model for solvent calculations and a QM/MM approach in order to model the aggregate systems, using the PBE0-D3 functional. The calculations find that the absorption behavior of the molecules in both phases is largely the same; only the emission is affected. This is found to arise from the fact that the molecules are unable to fully relax in the aggregate phase compared to the solution phase, resulting in a smaller Stokes' shift and blue-shifted emission.

Adamska $e a^{265}$ study a set of intriguing cycloparaphenylenes (CPP), highly conjugated ring systems that exhibit a ring-size independency with respect to absorbance maxima, and exhibit a ring-size dependency with respect to extinction coefficient. Fluorescence of these molecules is also found to be ring-size dependent, with a strong red-shift with respect to ring-size. CAM-B3LYP was used in order to study the absorbance of 8 different sized CPP hoops, finding across all of the molecules studied that the lowest energy transition results in a nodeless $S_{1}$ state that is delocalized across the entire structure which, due to the circular nature of the systems, results in no net transition dipole. The $\mathrm{S}_{2}$ and $\mathrm{S}_{3}$ states are found to be degenerate in even-numbered ring systems and quasi-degenerate in odd-numbered ring systems; both states have nodes and therefore exhibit significant transition dipole moments and therefore high spectroscopic absorbance. The increasing absorbance with respect to ring size is attributed to the increase in conjugation found in such systems. The ring-size independency with respect to 
absorbance maxima is found to arise from the near-exact cancellation of two competing effects. Increasing the ring size would result in a red-shift arising from increased conjugation as well as a decrease in the splitting between the $\mathrm{S}_{1}$ and $\mathrm{S}_{2} / \mathrm{S}_{3}$ states; however, increasing the ring size would also serve to blue-shift the absorbance due to a decrease in favorable through-space $\pi$-interactions (pseudo $\pi$-stacking) between neighbouring phenyl groups. The authors also employ non-adiabatic dynamics simulations in order to study the behavior of the molecules upon excitation, using PES generated on-the-fly by TD-DFT. These calculations demonstrate that the fluorescence observed for the larger CPP systems is due to strong vibronic coupling that results in a spatial localization of an exciton.

\subsection{Other properties}

Johnson et al. ${ }^{267}$ tackle the ability of DFT methods to accurately compute both first-order hyperpolarizabilities $(\beta)$ and absorption values, as large $\beta$ values give rise to efficient, high-bandwidth electro-optical devices and therefore accurate modelling of these systems will allow for their optimization. The authors focus on hybrid DFT functionals, i.e. those that incorporate some amount of $\mathrm{HF}$ exchange, finding that those with $<50 \% \mathrm{HF}$ exchange were unable to accurately reproduce $\beta$ values, whereas those with $>50 \%$ HF exchange provided better estimates of $\beta$ with, however, significantly blue-shifted absorbance energies. The authors suggest MP2 for $\beta$ calculations, and find that CAM-B3LYP and M06-2X were the most successful of the functionals tested in terms of accuracy.

Pescitelli and Bruhn ${ }^{268}$ give a review on good practice toward the computation of electronic circular dichroism spectra in molecules, and is also reviewed by Longhi et al. ${ }^{269}$ Ferrer et al. ${ }^{270}$ discuss the approach to first-principle simulation of vibrationally resolved absorption and emission spectra that include temperature and solvent effects.

Rozzi et al. ${ }^{271}$ combined femtosecond spectroscopy and TD-DFT to study the dynamics of a carotene-porphyrin-fullerene triad, in order to examine quantum coherence effects that could be exploited in artificial photosynthesis. The Perdew-Zunger functional ${ }^{272}$ (PZ81) was employed which uses the local density approximation (LDA), and should be suitable given the lack of charge-transfer character exhibited by the excited states of interest. The study finds that PZ81 was able to reproduce the Soret band and to some extent the Q-band of the porphyrin, once the calculated spectrum was red-shifted by $180 \mathrm{meV}$. The semi-classical dynamic simulations, where 
the nuclei are modelled as classical particles, find that almost complete charge-flow from the porphyrin to the fullerene takes place in approximately $70 \mathrm{fs}$, in good agreement with the experimentally observed timescale. In other studies, Santhanamoorthi et al. ${ }^{273}$ and Karthikeyan et al. ${ }^{274}$ used B3LYP with the 6-31G(d) basis set in order to investigate the effects of different sensitizers on the absorption behavior of porphyrin-based dyes.

Guido et al. ${ }^{275}$ benchmarked the performance of the B3LYP, CAMB3LYP, and PBE0 functionals in determining relaxed gas- and solventphase excited state geometries, compared to RI-CC2. The chromophores chosen in the test set exhibit both $n \pi^{*}$ and $\pi \pi^{*}$ transitions, and PCMacetonitrile solvation is used. Adiabatic energies are not considered. The B3LYP and PBE0 functionals are found to perform best for vertical excitation energy and ground-state structure; however, CAM-B3LYP is found to perform best for excited state geometries.

Sousa et al. ${ }^{276}$ coupled TD-DFT and CASSCF/CASPT2 to study the ultrafast deactivation mechanism of $\left[\mathrm{Fe}(2,2 \text {-bipyridine })_{3}\right]^{2+}$. The TD-DFT calculations with the PBE0 functional were used to calculate the geometries and frequencies of the ground and excited states of the different spin states of the complex, and CASPT2 calculations were used for the ultimate energy comparisons and SOC computations. This combined approach suggests a 5 -step deactivation process that involves singlet $\rightarrow$ triplet intersystem crossing, triplet $\rightarrow$ triplet internal conversion, with a final triplet $\rightarrow$ quintuplet intersystem crossing.

Cerezo et al. ${ }^{277}$ studied the issue of coordinate choice when modelling vibrationally resolved absorption spectra. The excited state PES were constructed using the adiabatic $(\mathrm{AH})$ and vertical Hessian $(\mathrm{VH})$ models, with internal coordinates and Cartesian coordinates used with $\mathrm{AH}$. The study finds that, when the $\mathrm{S}_{0} \rightarrow \mathrm{S}_{1}$ transition results in significant geometric displacement, Cartesian coordinates perform poorly for absorption spectra due to being unable to cope with curvilinear displacements; using internal coordinates alleviates this issue. The $\mathrm{VH}$ approach is found to generally give good agreement between experimental and computed spectra independent of the extent of relaxation in the excited state.

Long et al. ${ }^{278}$ designed a coumarin-based fluorescent probe capable of detecting biological thiols. The initial compound is found to exhibit almost no fluorescence in solution, and upon reaction with a thiol fluorescence is switched on. B3LYP is used to determine the extent of the CT for the different forms of the probe, finding that CT, and therefore fluorescence, is inhibited in the non-thiol-reacted form. However, as B3LYP fails with CT states it's unclear to what extent this is true. 
Baiardi et al. ${ }^{279}$ present the implementation of a time-independent model that can include Franck-Condon, Herzberg-Teller, and Duchinsky effects $^{280}$ in the simulation of vibrationally resolved absorption spectra. The authors note the importance of simulating electronic transitions when attempting to assign experimental spectra, but also highlight how, generally, the simulation of spectra is limited to the simple fitting of a symmetric Gaussian function to the absorption maximum. This in turn limits the amount of information that can be extracted from the calculations and can prevent them from truly complementing experimental results. The authors therefore developed this vibronic model in order to enhance excited state calculations. The BLYP functional was employed against a test set of molecules that included anthracene, furan, phenyl radical, $\mathrm{R}, R$ dimethyloxirane, and coumarin 339. The resulting spectra exhibit significant vibrational fine structure in good agreement with experimentally obtained photoionization and absorption spectra.

Mitri et al. ${ }^{281}$ employed a QM/MD approach to simulating the absorption and emission spectrum of 4-naphthoyloxy-1-methoxy-2,2,6,6tetramethyl-piperidine (NfO-TEMPO-Me) in toluene; the MD calculations were run for $3 \mathrm{~ns}$, frames of which were used to build an average UV-vis spectrum as calculated with CAM-B3LYP and PBE0. The MD approach was employed due to the flexibility of the NfO-TEMPO system, which can occupy a range of spectroscopically relevant equilibrium structures at the temperature of $300 \mathrm{~K}$. The calculations find good agreement with experimental data, when comparing vertical excitation energies, Stoke's shift, and solvatochromic shifts.

Li et al. have applied TD-DFT to open-shell systems ${ }^{282-285}$ and to the effect of solvent on the excited states of triphenylamine-thiadiazole (TPANZP). ${ }^{286}$ In particular, for the open-shell systems, which often suffer from spin-contamination issues, the spin-adapted (X-) TD-DFT method was found to cope well and offer an increase in accuracy for doublet-doublet and doublet-quartet transitions. In the case of TPA-NZP, the $\mathrm{S}_{0}$ geometries were found to be solvent independent, and the $S_{1}$ geometry was found to be solvent dependent. The study also finds that increasing the solvent polarity increases the amount of CT in the $\mathrm{S}_{1}$ state. However, as the excited states were computed with B3LYP, it is unclear to what extent the CT really dominates.

\subsection{Assessment}

TD-DFT methods are certainly the most widely used in photochemical studies as they offer the best compromise between accuracy and 
Table 3 TD-DFT summary.

Property

Trends

Quantitative

\begin{tabular}{lll}
\hline Nonlinear optical properties & TD-HF & TD-HF $^{\mathrm{a}}$ \\
\hline Absorption spectra & Yes $^{\mathrm{b}}$ & Yes $^{\mathrm{b}}$ \\
\hline Fluorescence spectra & Yes $^{\mathrm{b}}$ & Yes $^{\mathrm{b}}$ \\
\hline Photochemical mechanisms & Sometimes $^{\mathrm{b}}$ & No \\
\hline Quantitative photochemical Kinetics & No & No \\
\hline
\end{tabular}

${ }^{a}$ Depends on the system and whether it is a first, second, third order hyperpolarizability, with higher order polarizabilities modelled better than lower order ones.

${ }^{b}$ Depends on system and choice of functional. In particular, states involving charge transfer character need to be treated with a long-range corrected functional, relaxed densities are needed for emission spectra and modern functional with improved correlation/dispersion treatment tend to perform better.

computational cost. They are also much simpler to use than the more accurate multireference methods, which in turn require careful selection of active space orbitals. The main applications of TD-DFT are in the prediction of absorption and emission spectra, and their accuracy depends upon the system and choice of functional (Table 3). As a general rule, systems involving significant charge-transfer character require long-range corrected functionals, but even these are not always sufficiently accurate. Owing to its low cost, TD-DFT is also frequently used to study photochemical reaction mechanisms such as excited state intermolecular proton transfer, photoinitiation, and photoisomerization. Often it is able to qualitatively explain experimental results, but does have failures and is rarely used for quantitative studies. However, new developments such as Truhlar's Dual-Functional Tamm-Dancoff Approximation ${ }^{153}$ show enormous promise in raising the accuracy of TD-DFT to that of equivalent multireference methods, but at a fraction of the cost. At the other extreme, Grimme's Simplified Tamm-Dancoff Approximation has recently been shown to reproduce standard TD-DFT at a fraction of the cost, allowing systems with 1000s of atoms to be studied. ${ }^{154}$

\section{Solvent and environmental effects \\ 7.1 Continuum versus explicit solvent}

Solvent effects can have profound influences on the excited state behavior, which can be difficult to fully capture. Implicit solvent effects remain popular; however, they will fail, when explicit solvent-solute bonding is 
taking place. For example, hydrogen bonding of water to a carbonyl oxygen will result in the blue-shift in the corresponding $n \pi^{*}$ transition, due to stabilisation of the nonbonding oxygen electrons, and implicit solvent models will therefore be inherently inaccurate when considering such an effect.

Including explicit solvent molecules can add to the cost of already expensive calculations. To address this, Zhang et al. ${ }^{287}$ have implemented a local excitation approximation (LEA) for CIS, TD-HF, and TD-DFT, in which an optically active region (containing the chromophore) is defined in a QM/ $\mathrm{MM}$, chromophore/solvent system. Using local excitation serves to truncate excited state calculations as most of the explicit solvent molecules are not considered. However, the method benefits relative to simply using the polarizable continuum models (PCM), as important solvent-chromophore interactions are still included. The LEA-TD-DFT implementation results in a speedup of 6-10 times for calculations, compared with a standard TD-DFT calculation for the same system. The LEA schemes for all three of the tested methods also show significant blue-shifting of the $n \pi^{*}$ transition observed for aqueous acetone, compared with gas-phase acetone, suggesting the LEA scheme is correctly capturing explicit solvent-chromophore interactions. However, no comparison to experimental data is provided, so it is unclear if the LEA-TD approach is outperforming traditional PCM-TD approaches.

Milanese et al. ${ }^{288}$ studied the convergence of excited state calculations with respect to increasing the number of explicit water molecules solvating four 1,4-diphenylbuta-1,3-diene derivatives in a full QM approach, from 0 to 400 water molecules, citing concerns with defining the QM/MM regions as to why such a system was not used. TD-DFT and CIS methods are compared with several basis sets $\left(3-21 G, 6-31 G, 6-31 G^{*}, 6-31+G^{*}\right)$, and the emphasis is on the TD-(LC-) $\omega \mathrm{PBE}$ results. The addition of polarization functions, from 6-31G to $6-31 \mathrm{G}^{*}$, was found to have an insignificant effect on the absorption spectra, however, increasing the size of the basis set, from 3-21G to $6-31 \mathrm{G}$, then to the $6-31+\mathrm{G}^{*}$ results in a concomitant decrease in the HOMO-LUMO transition energy. The biggest change in transition energy, for all basis sets with CIS and TD-DFT approaches, arises in approximately the $0-100$ water molecule regime. The authors note that CIS convergence with respect to explicit solvation is faster than that of TD-DFT, and that increasing the size of the basis set also results in faster convergence. Clearly, however, there is a balance, between increasing the number of solvating molecules and increasing the basis set size, with respect to computational expense. 
Like that of Zhang, ${ }^{287}$ neither experimental nor PCM-TD absorption spectra are included for comparison, so convergence with respect to physical relevance/computational expense is not quantified. Such a comparison would also allow for better estimation of the required size of a QM region of solvated chromophore, and therefore whether a full QM, rather than a $\mathrm{QM} / \mathrm{MM}$, treatment is required.

The contrast between implicit and explicit solvation is illustrated in studies by Martínez-Fernández et al. ${ }^{289}$ and Szabla et al. ${ }^{290}$ of the absorption properties of methylated cytosine derivatives. Martínez-Fernández et al. used TD-CAM-B3LYP and CASPT2/MM to describe how the low-lying $\pi \pi_{1}^{*}$, $\pi \pi_{2}^{*}, n_{\wedge} \pi^{*}$, and $n_{\mathrm{O}} \pi^{*}$ are vibronically coupled, and therefore population of the dark states is possible. These states are then able to decay to the ground state, and the excited state lifetimes are estimated using the CASPT2/MM approach. However, Szabla et al. argued that the approach by Martínez-Fernández et al. is insufficient to properly describe the experimentally observed behavior of the cytosine/cytidine derivatives, as the CASPT2/MM calculations do not include any explicit solvent molecules in the QM region, and the TD-DFT calculations utilize the nonexplicit PCM solvent model. In their study, Szabla et al. find that microsolvation of the N1-methylcytosine by two $\mathrm{H}_{2} \mathrm{O}$ molecules results in a dark, low-lying water-to-chromophore charge transfer state, denoted $n_{N} \pi_{C T}^{*}$, and significant destabilization of the $n_{\mathrm{O}} \pi^{*}$ state to approximately $3 \mathrm{eV}$ above the CT state. Exploring the PES of the $n_{N} \pi_{C T}^{*}$ state demonstrates the possibility for an electron-driven proton transfer (EDPT), along the coordinate of which there is a conical intersection between the $S_{1}$ and $S_{0}$ state, which forms a reactive hydroxyl anion that could further react to form uracil derivatives.

Li et al. ${ }^{291}$ also studied the effect of explicit solvent molecules on the absorption spectrum of cytosine, using a QM/MM approach. The structure of the solute is determined using CASSCF, upon which CASPT2 vertical excitation energies are calculated. The approach is found to blue-shift the $n \pi^{*}$ transitions, relative to values calculated with PCM, a result that is to be expected as the inclusion of explicit solvent-solute hydrogen-bonds stabilises the $n$-orbitals. There is also, however, significant deviation observed for some of the $\pi \pi^{*}$ transitions; this is likely due to the CASSCF calculated geometries not being accurate enough. Spata and Matsika ${ }^{292}$ studied a conical intersection in a 9-methyladenine homodimer, finding that, upon excitation to a low-lying $\pi \pi^{*}$ state significant charge-transfer takes place between the two adenine species. Radiationless decay can also take place between the $S_{0}$ and $S_{1}$ states, and higher energy excitations result in the population of the dissociative part of the $\mathrm{S}_{0}$ PES. 
Fang and $\mathrm{Kim}^{293}$ used (MRPT2-)CASSCF to study the excited state tautomerization of 7-azaindole complexed with a methanol molecule; the tautomerization involves transfer of a hydrogen atom to the methanol molecule, before a subsequent $\mathrm{H}$-transfer from the methanol to a neighboring nitrogen atom. The rates of transfer were calculated with variational transition state theory, and it is also found that the integral equation formalism PCM (IEFPCM) solvent model was not capable of reproducing the correct energetics of the reaction.

Zuehlsdorff et al. ${ }^{294,295}$ have studied the effect of including explicit solvent molecules on the simulated absorption properties of Nile Red and alizarin. The study of alizarin used a QM/MM model (TD-PBE and TD-CAM-B3LYP for the QM region) and found a relatively slow convergence of absorption accuracy vs number of explicit solvent molecules, with up to 380 water molecules being required for accurate results. The slow convergence was attributed to charge transfer to solvent molecules in the immediate vicinity of the alizarin molecule, and long-range electrostatic effects of the water molecules. The study of Nile Red found that the failure of implicit solvent models to describe $\pi$-stacking and $\mathrm{H}$-bonding interactions led to significant deviations in calculated absorption peaks. The study also found that the PBE functional systematically underestimated excitation energies, and that CAM-B3LYP could be used to improve the results.

Chang et al. ${ }^{296}$ studied the photoprotection mechanism of p-methoxy methylcinnamate, which was found to display increased radiationless decay to the $\mathrm{S}_{0}$ state upon addition of water. CASPT2-corrected CASSCF calculations demonstrate that initial excitation is to $\mathrm{a}^{1} \pi \pi^{*}$, which can undergo either internal conversion to $a^{1} n \pi$ state, or deactivation to the $\mathrm{S}_{0}$ state via a conical intersection along the photoisomerization pathway. The $\mathrm{H}_{2} \mathrm{O}$ explicit solvent molecule is found to stabilize the $\pi \pi^{*}$ state and destabilise the $n \pi^{*}$ state, compared with the unsolvated species, resulting in an increase in favorability for the photoisomerization deactivation pathway to take place.

Charlton et al. ${ }^{297}$ used several flavors of the PBE functional including the LC- $\omega$ PBE functional, the optimally tuned (OT-) LC- $\omega$ PBE, and the PBE functional itself, to study the effect of including explicit, near-neighbour, nitrogen-doped pentacene molecules around a single pentacene molecule. The use of the long-range corrected functionals was to negate some of the error associated with charge-transfer excited states, which were being modeled. The study found that the explicit inclusion of the surrounding molecules resulted in a red-shift in the $S_{1}$ state, compared to values calculated with a PCM solvent model. 
Marazzi et al. ${ }^{298}$ used TD-DFT and CASPT2 to calculate the electronic properties of Nile red and Nile blue chromophores. The authors also employed two types of geometry upon which vertical excitation energies were calculated; that is, QM optimized $\mathrm{S}_{0}$ equilibrium structures, and dynamic, explicitly solvated structures as calculated with MD. The dynamic structures were found to give red-shifted energies compared with the static structures and are in closer agreement with experimental data.

Mewes et al. ${ }^{299}$ reported the implementation and performance of a correction scheme for vertical excitation energies calculated with $\mathrm{ADC}(\mathrm{n})$ and TD-DFT (LRC- $\omega$ PBE) in a PCM solvent environment, using a test set of molecules mainly made up of nitroaromatic compounds. The correction is possible with both linear-response (LR-PCM) and state-specific (SS-PCM) PCM schemes. At best, the study finds that the correction scheme is able to reproduce experimental solvent shifts, when the shifts arise from bulk electrostatic interactions, with maximum absolute errors and mean absolute deviations of $50 \mathrm{meV}$ and $20-30 \mathrm{meV}$, respectively. The authors also find that the success of LR-PCM vs SS-PCM is dependent on the extent of orbital relaxation included in the excited-state calculation; methods that do not include orbital relaxation, i.e., TD-DFT/ADC(1), performed best with LR-PCM (essentially unrelaxed PCM), whereas higher-order $\mathrm{ADC}(\mathrm{n})$ calculations performed best with SS-PCM.

Isborn et al. ${ }^{300}$ studied the issues when using TD-DFT with chromophores explicitly solvated by water molecules to explore QM/MM calculations that often result in the spurious, low-energy solvent $\rightarrow$ chromophore (or vice versa) charge-transfer states. These charge-transfer artefact states are found to arise from so-called "edge" water molecules, molecules that are hydrogen-bond unsaturated and therefore have high-lying HOMOs. The effects of the edge waters are exacerbated by small basis sets, unoptimized geometries, and when using functionals that do not contain any exact HF exchange. However, the LC- $\omega \mathrm{PBEh}$ functional, which is both long-range corrected and incorporates $20 \%$ HF exchange, is found to reduce the impact of the edge water molecules and gives low-energy excitations corresponding to the optically relevant chromophore valence states.

\subsection{Equilibrium versus nonequilibrium solvation}

Molecules in their excited states are dynamic systems, exhibiting different phenomena that take place over a range of timescales, ${ }^{301}$ and different components of the model system exhibit different response timescales to each other. 
This gives rise to "nonequilibrium" solvation effects, in which the redistribution of electron density upon photon absorption is assumed to be faster than solvent nuclei redistribution. Vertical excitation energies, i.e., the instantaneous redistributions of electron density, are therefore calculated using nonequilibrium solvation, whereas relaxed properties, for example, excited state geometry optimizations, are calculated using equilibrium solvation. In calculations including explicit solvent effects, nonequilibrium vertical excitation energies are achieved simply by taking single-point energies. In continuum solvent models this is generally achieved by separating the solvent response into a dynamic component, which is relaxed alongside the new solute electron density, and an inertial component, which is kept frozen in equilibrium with the initial state of the molecule. ${ }^{302}$

$\mathrm{Li}^{303}$ provides a comprehensive overview of continuum models for nonequilibrium solvation. Generally, however, studies explicitly dealing with nonequilibrium versus equilibrium solvation effects do so with respect to comparing state-specific and linear-response formalisms for excited state calculations. ${ }^{302,304-306}$

\subsection{Other environmental effects}

Chromophores involving lone pairs (e.g., on oxygen or nitrogen) undergo $n \pi^{*}$ transitions. These can be disrupted by protonating the lone pair through $\mathrm{pH}$ changes. Likewise, if conjugated with a $\pi$ system, the stability of the $\pi$ and $\pi^{*}$ orbitals can also change dramatically the protonation state of acidic or basic functional groups. These $\mathrm{pH}$ effects can be studied conveniently in silico by considering the charged and neutral forms. This approach can be used in conjunction with experimental spectroscopic data to help ascertain the protonation state of a species, particularly in environments (such as enzymes) where direct titration is difficult. ${ }^{8}$ In a similar vein, complexation with Lewis acids has been shown to dramatically alter the photochemical behavior of photoinitiators, blue shifting the $n \pi^{*}$ excitations of type I photoinitiators, methyl-4'-(methylthio)-2-morpholinopropiophenone (MMMP) and 2,2dimethoxy-2-phenylacetophenone (DMPA), while concurrently red shifting the $\pi \pi^{*}$ transitions. ${ }^{1}$

Even when direct conjugation is not possible, the electrostatic effect of charged functional groups can also influence the relative energies of excited states due to their different polarities. Recently Hill and Coote showed used TD-DFT that by including these charged functional groups as nonconjugated substituents, simple $\mathrm{pH}$ changes can be used to 
selectively modify the relative energies (and even ordering) of the $n \pi^{*}$ and $\pi \pi^{*}$ states of an acetophenone derivative. ${ }^{307}$ This proof-of-concept study suggests significant opportunities to selectively tune photochemical reactivity.

\section{Conclusion}

While photochemical processes are challenging to model using computational methods, much progress has been made in this field over recent years. Expensive multireference calculations remain the gold standard for studying photochemical reactions, particularly on nonadiabatic surfaces (Fig. 4). However, improvements to TD-DFT, such as long range corrections, dispersion corrections and, most recently, the Dual-Functional Tamm-Dancoff Approximation, ${ }^{153}$ has meant that are they are starting to rival multireference in accuracy even for excited state potential energy surfaces. More generally, TD-DFT has become routine for the prediction of absorption and emission spectra, and in most cases provides qualitatively if not quantitatively useful results. For large systems, the wavefunction based TD-HF and CIS methods have traditionally been the only option, providing useful predictions of nonlinear optical properties but generally failing for other photochemical phenomena. However, the use of methods based on localized orbitals (for correlated wavefunctions), and the Simplified Tamm-Dancoff Approximation ${ }^{154}$ and TD-DFT ${ }^{155}$ has made it possible to apply more accurate methods to increasingly larger systems. At the same time the use of QM/MM methods have also made it possible to provide increasingly accurate treatments of solvent effects on photochemical processes without the prohibitive cost of including explicit solvent molecules in the excited state QM treatment.

The value of these methodological improvements is evident in the wide range of applications highlighted herein. Theory has proven extremely useful in clarifying photochemical reaction mechanisms and, for example, showing that targeting dark states sometimes provides more efficient reactivity. Theoretical studies have helped to provide an insight into structure-reactivity trends in a range of photochemical phenomena, in turn providing guidelines for optimizing nonlinear optical properties, dye-sensitized solar cells, redoxresponsive fluorescent probes and visible light photoinitiators, among other applications. Moreover, the recent demonstration of electrostatic tuning of photochemical reactivity may open up yet further avenues for computational design in this field. 

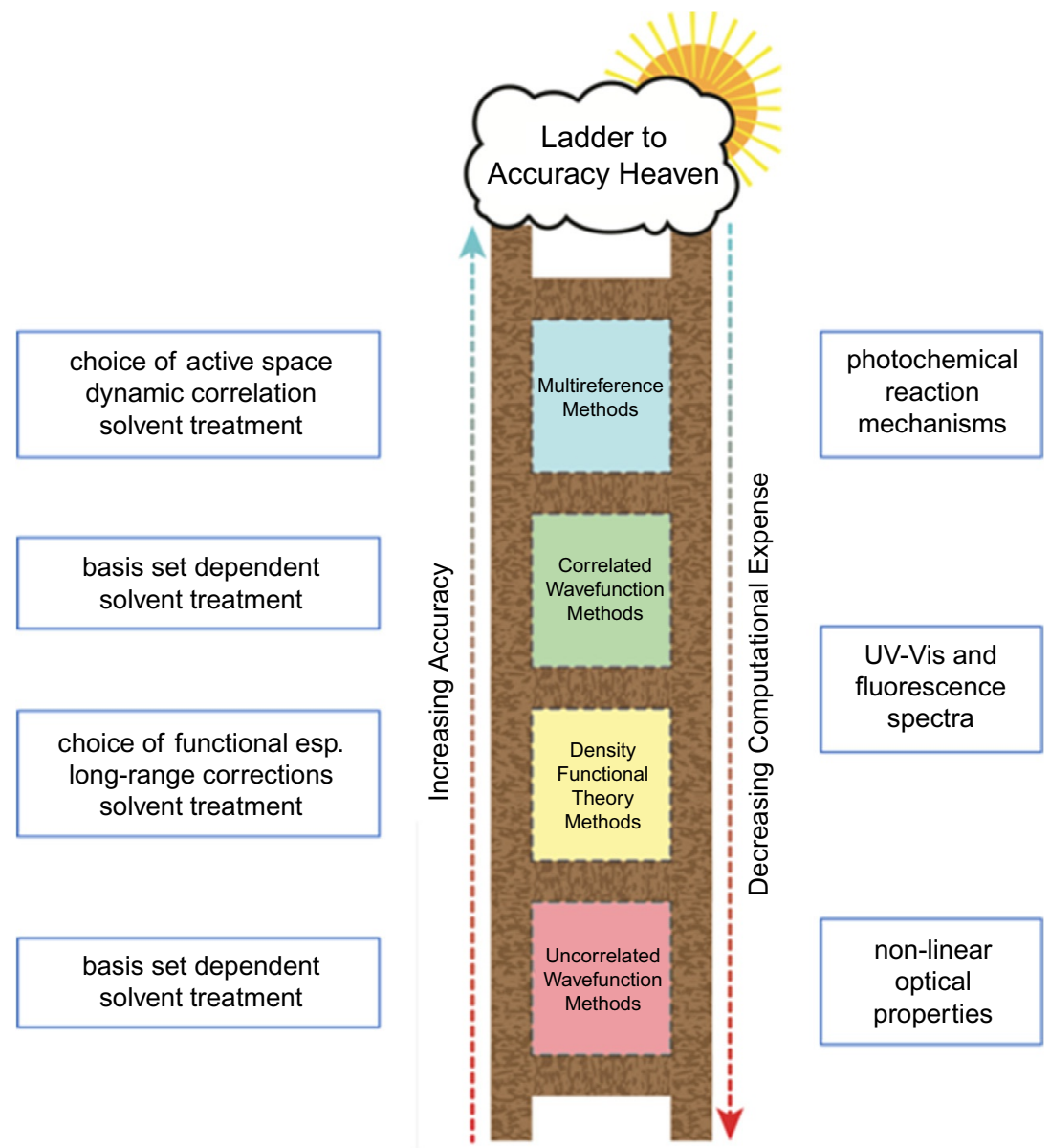

Fig. 4 Guidelines for selecting theoretical methods.

\section{Conflicts of interest}

There are no conflicts to declare.

\section{Acknowledgments}

The authors acknowledge financial support from the Australian Research Council (ARC: CE140100012, FL170100041) Centre of Excellence for Electromaterials Science, and an ARC Laureate Fellowship (to M.L.C.).

\section{References}

1. Raghavachari, K.; Anderson, J. B. Electron Correlation Effects in Molecules. J. Phys. Chem. 1996, 100(31), 12960-12973.

2. Santoro, F.; Jacquemin, D. Going Beyond the Vertical Approximation With Time-Dependent Density Functional Theory. Wiley Interdiscip. Rev. Comput. Mol. Sci. 2016, 6(5), 460-486. 
3. Improta, R.; Santoro, F.; Blancafort, L. Quantum Mechanical Studies on the Photophysics and the Photochemistry of Nucleic Acids and Nucleobases. Chem. Rev. 2016, 116(6), 3540-3593.

4. Laurent, A. D.; Jacquemin, D. TD-DFT Benchmarks: A Review. Int. J. Quantum Chem. 2013, 113(17), 2019-2039.

5. Garavelli, M. Computational Organic Photochemistry: Strategy, Achievements and Perspectives. Theor. Chem. Acc. 2006, 116(1-3), 87-105.

6. Serrano-Andrés, L.; Merchán, M. Quantum Chemistry of the Excited State: 2005 Overview. J. Mol. Struct. Theochem 2005, 729, 99-108.

7. Menzel, J. P.; Noble, B. B.; Lauer, A.; Coote, M. L.; Blinco, J. P.; Barner-Kowollik, C. Wavelength Dependence of Light-Induced Cycloadditions. J. Am. Chem. Soc. 2017, 139(44), 15812-15820.

8. Mohamed, A. E.; Ahmed, F. H.; Arulmozhiraja, S.; Lin, C. Y.; Taylor, M. C.; Krausz, E. R.; Jackson, C. J.; Coote, M. L. Protonation State of F420H2in the Prodrug-Activating Deazaflavin Dependent Nitroreductase (Ddn) From Mycobacterium Tuberculosis. Mol. Biosyst. 2016, 12(4), 1110-1113.

9. Li, H. P.; Bi, Z. T.; Xu, R. F.; Han, K.; Li, M. X.; Shen, X. P.; Wu, Y. X. Theoretical Study on Electronic Polarizability and Second Hyperpolarizability of Hexagonal Graphene Quantum Dots: Effects of Size, Substituent, and Frequency. Carbon N. Y. 2017, 122, 756-760.

10. Noble, B. B.; Mater, A. C.; Smith, L. M.; Coote, M. L. The Effects of Lewis Acid Complexation on Type I Radical Photoinitiators and Implications for Pulsed Laser Polymerization. Polym. Chem. 2016, 7, 6400.

11. Zhang, J.; Lalevée, J.; Hill, N. S.; Launay, K.; Morlet-Savary, F.; Graff, B.; Stenzel, M. H.; Coote, M. L.; Xiao, P. Disubstituted Aminoanthraquinone-Based Multicolor Photoinitiators: Photoinitiation Mechanism and Ability of Cationic Polymerization under Blue, Green, Yellow, and Red LEDs. Macromolecules 2018, 51, 8165-8173.

12. Blancafort, L. Photochemistry and Photophysics at Extended Seams of Conical Intersection. ChemPhysChem 2014, 15(15), 3166-3181.

13. Polo, V.; Kraka, E.; Cremer, D. Electron Correlation and the Self-Interaction Error of Density Functional Theory. Mol. Phys. 2002, 100(11), 1771-1790.

14. Karakas, A.; Karakaya, M.; Taser, M.; Ceylan, Y.; Gozutok, A.; Arof, A. K.; El Kouari, Y.; Sahraoui, B. Computational Studies on Linear, Second and Third-Order Nonlinear Optical Properties of Novel Styrylquinolinium Dyes. Chem. Phys. Lett. 2016, 648, 47-52.

15. Zawadzka, A.; Karakas, A.; Płóciennik, P.; Szatkowski, J.; Łukasiak, Z.; Kapceoglu, A.; Ceylan, Y.; Sahraoui, B. Optical and Structural Characterization of Thin Films Containing Metallophthalocyanine Chlorides. Dye. Pigment. 2015, 112, 116-126.

16. Karakas, A.; Dag, T.; El Ouazzani, H.; Sebiaai, K.; Boughaleb, Y. Two-Photon Absorption and Third-Order Nonlinear Optical Characterization of PyrroloTetrathiafulvalene Derivatives. Opt. Quantum Electron. 2014, 46(1), 165-170.

17. Guezguez, I.; Karakas, A.; Iliopoulos, K.; Derkowska-Zielinska, B.; El-Ghayoury, A.; Ranganathan, A.; Batail, P.; Migalska-Zalas, A.; Sahraoui, B.; Karakaya, M. Theoretical and Experimental Investigations on the Nonlinear Optical Properties of Gold(III) Dithiolene Complexes. Opt. Mater. (Amst). 2014, 36(1), 106-111.

18. Wu, J.; Wilson, B. A.; Smith, D. W., Jr.; Nielsen, S. O. Towards an Understanding of Structure-Nonlinearity Relationships in Triarylamine-Based Push-Pull Electro-Optic Chromophores: The Influence of Substituent and Molecular Conformation on Molecular Hyperpolarizabilities. J. Mater. Chem. C 2014, 2(14), 2591.

19. Alparone, A. Nonlinear Optical Properties of Fluorobenzenes: A Time-Dependent Hartree-Fock Study. Comput. Theor. Chem. 2013, 1013, 23-24. 
20. Bondu, F.; Quertinmont, J.; Rodriguez, V.; Pozzo, J.-L.; Plaquet, A.; Champagne, B.; Castet, F. Second-Order Nonlinear Optical Properties of a DithienyletheneIndolinooxazolidine Hybrid: A Joint Experimental and Theoretical Investigation. Chem. A Eur. J. 2015, 21(51), 18749-18757.

21. Brückner, C.; Walter, C.; Stolte, M.; Braida, B.; Meerholz, K.; Würthner, F.; Engels, B. Structure-Property Relationships for Exciton and Charge Reorganization Energies of Dipolar Organic Semiconductors: A Combined Valence Bond SelfConsistent Field and Time-Dependent Hartree-Fock and DFT Study of Merocyanine Dyes. J. Phys. Chem. C 2015, 119(31), 17602-17611.

22. Hidalgo Cardenuto, M.; Champagne, B. QM/MM Investigation of the Concentration Effects on the Second-Order Nonlinear Optical Responses of Solutions. J. Chem. Phys. 2014, 141(23), 234104.

23. Tamer, Ö.; Gözüaç1k, F.; Avc1, D.; Atalay, Y. Theoretical Analysis on Structural, Spectroscopic, and Electronic Properties of Some 2-Aminobenzimidazole Complexes by Using PBE1PBE, B3LYP, and HF Methods. Opt. Spectrosc. 2014, 116(1), 12-32.

24. Maidur, S. R.; Patil, P. S.; Rao, S. V.; Shkir, M.; Dharmaprakash, S. M. Experimental and Computational Studies on Second-and Third-Order Nonlinear Optical Properties of a Novel D- $\pi$-A Type Chalcone Derivative: 3-(4-Methoxyphenyl)-1(4-Nitrophenyl) Prop-2-En-1-One. Opt. Laser Technol. 2017, 97, 219-228.

25. Lötstedt, E.; Kato, T.; Yamanouchi, K. Intramolecular Electron Dynamics in the Ionization of Acetylene by an Intense Laser Pulse. J. Chem. Phys. 2013, 138(10), 104304.

26. Hu, S.-L.; Zhao, Z.-X.; Chen, J.; Shi, T.-Y. Ionization Dynamics of C2H2 in Intense Laser Fields: Time-Dependent Hartree-Fock Approach. Phys. Rev. A 2015, 92(5) 053409 .

27. Luzanov, A. V. Nonstationarity and Related Measures for Time-Dependent HartreeFock and Multiconfigurational Models. Int. J. Quantum Chem. 2013, 113(23), 2489-2505.

28. Nguyen, T. S.; Parkhill, J. Nonadiabatic Dynamics for Electrons at Second-Order: Real-Time TDDFT and OSCF2. J. Chem. Theory Comput. 2015, 11(7), 2918-2924.

29. Sato, T.; Ishikawa, K. L. The Structure of Approximate Two Electron Wavefunctions in Intense Laser Driven Ionization Dynamics. J. Phys. B At. Mol. Opt. Phys. 2014, 47(20), 204031.

30. Tanak, H.; Toy, M. Molecular Structure and Vibrational Assignment of 1-[N-(2-Pyridyl) Aminomethylidene]-2(1H)-Naphtalenone by Density Functional Theory (DFT) and $\mathrm{Ab}$ Initio Hartree-Fock (HF) Calculations. Spectrochim. Acta A Mol. Biomol. Spectrosc. 2016, 152, 525-529.

31. Dreuw, A.; Head-Gordon, M. Single-Reference Ab Initio Methods for the Calculation of Excited States of Large Molecules. Chem. Rev. 2005, 105(11), 4009-4037.

32. Subotnik, J. E. Communication: Configuration Interaction Singles Has a Large Systematic Bias against Charge-Transfer States. J. Chem. Phys. 2011, 135(7), 071104.

33. Cheng, C. Y.; Ryley, M. S.; Peach, M. J. G.; Tozer, D. J.; Helgaker, T.; Teale, A. M. Molecular Properties in the Tamm-Dancoff Approximation: Indirect Nuclear SpinSpin Coupling Constants. Mol. Phys. 2015, 113(13-14), 1937-1951.

34. Nithya, R.; Sowmiya, M.; Kolandaivel, P.; Senthilkumar, K. Structural, Optical, and Charge Transport Properties of Cyclopentadithiophene Derivatives: A Theoretical Study. Struct. Chem. 2014, 25(3), 715-731.

35. Cebrián, C.; De Mata Muñoz, J.; Strassert, C. A.; Prieto, P.; Díaz-Ortiz, Á.; De Cola, L.; De La Hoz, A. Synthesis of Bright Alkenyl-1H-1,2,4-Triazoles: A Theoretical and Photophysical Study. Chempluschem 2014, 79(10), 1489-1497.

36. Tathe, A. B.; Gupta, V. D.; Shreykar, M. R.; Ramasami, P.; Sekar, N. Excited State Intramolecular Proton Transfer of 2-(2',6'-Dihydroxyphenyl)Benzoxazole: Insights Using Computational Methods. J. Lumin. 2014, 154, 267-273. 
37. Laurent, A. D.; Blondel, A.; Jacquemin, D. Choosing an Atomic Basis Set for TD-DFT, SOPPA, ADC(2), CIS(D), CC2 and EOM-CCSD Calculations of Low-Lying Excited States of Organic Dyes. Theor. Chem. Acc. 2015, 134(6), 1-11.

38. Aziz, S. G.; Alyoubi, A. O.; Elroby, S. A.; Hilal, R. H. Photochemical Dissociation of HOBr. A Nonadiabatic Dynamics Study. J. Photochem. Photobiol. A Chem. 2016, 324, $8-13$.

39. Shinde, R. Benchmarking Quantum Chemical Methods for Optical Absorption in Boron Wheels. ACS Omega 2016, 1(4), 578-585.

40. Shayan, K.; Nowroozi, A. The First Singlet Excited State (S1) Intramolecular Hydrogen Bond of Malonaldehyde Derivatives: A TD-DFT and CIS Study. Struct. Chem. 2016, 27(6), 1769-1780.

41. Annaraj, B.; Mitu, L.; Neelakantan, M. A. Synthesis and Crystal Structure of Imidazole Containing Amide as a Turn on Fluorescent Probe for Nickel Ion in Aqueous Media. An Experimental and Theoretical Investigation. J. Mol. Struct. 2016, 1104, 1-6.

42. Kocherzhenko, A. A.; Sosa Vazquez, X. A.; Milanese, J. M.; Isborn, C. M. Absorption Spectra for Disordered Aggregates of Chromophores Using the Exciton Model. J. Chem. Theory Comput. 2017, 13(8), 3787-3801.

43. Farrokhpour, H.; Dehdashti Jahromi, M. Absorption Spectra of Small Helium Nano Clusters (4Hen; $\mathrm{n}=2-029$ ) and Characterization of Their Low-Lying Excited States. J. Mol. Liq. 2017, 230, 190-199.

44. Ge, Q.; Mao, Y.; White, A. F.; Epifanovsky, E.; Closser, K. D.; Head-Gordon, M. Simulating the Absorption Spectra of Helium Clusters ( $N=70,150,231,300)$ Using a Charge Transfer Correction to Superposition of Fragment Single Excitations. J. Chem. Phys. 2017, 146(4), 044111.

45. Krylov, A. I. Spin-Flip Configuration Interaction: An Electronic Structure Model That Is Both Variational and Size-Consistent. Chem. Phys. Lett. 2001, 350(5-6), 522-530.

46. Rhee, Y. M.; Head-Gordon, M. Scaled Second-Order Perturbation Corrections to Configuration Interaction Singles: Efficient and Reliable Excitation Energy Methods. J. Phys. Chem. A 2007, 111(24), 5314-5326.

47. Chibani, S.; Laurent, A. D.; Le Guennic, B.; Jacquemin, D. Improving the Accuracy of Excited-State Simulations of BODIPY and Aza-BODIPY Dyes with a Joint SOSCIS(D) and TD-DFT Approach. J. Chem. Theory Comput. 2014, 10(10), 4574-4582.

48. Chibani, S.; Laurent, A. D.; Le Guennic, B.; Jacquemin, D. Excited States of Ladder-Type $\pi$-Conjugated Dyes With a Joint SOS-CIS(D) and PCM-TD-DFT Approach. J. Phys. Chem. A 2015, 119(21), 5417-5425.

49. Ponce-Vargas, M.; Azarias, C.; Jacquemin, D.; Le Guennic, B. Combined TD-DFTSOS-CIS(D) Study of BOPHY Derivatives with Potential Application in Biosensing. J. Phys. Chem. B 2017, 121(48), 10850-10858.

50. Pulay, P. A Perspective on the CASPT2 Method. Int. J. Quantum Chem. 2011, 111, 3273-3279.

51. Sumita, M.; Morihashi, K. Theoretical Study of Singlet Oxygen Molecule Generation via an Exciplex with Valence-Excited Thiophene. J. Phys. Chem. A 2015, 119(5), 876-883.

52. Chang, X. P.; Fang, Q.; Cui, G. Mechanistic Photodecarboxylation of Pyruvic Acid: Excited-State Proton Transfer and Three-State Intersection. J. Chem. Phys. 2014, 141(15), 2-10.

53. Cui, G.; Guan, P. J.; Fang, W. H. Photoinduced Proton Transfer and Isomerization in a Hydrogen-Bonded Aromatic Azo Compound: A CASPT2//CASSCF Study. J. Phys. Chem. A 2014, 118(26), 4732-4739.

54. Casellas, J.; Reguero, M. Photosensitization Versus Photocyclization: Competitive Reactions of Phenylphenalenone in Its Role as Phytoanticipins in Plant Defense Strategies. J. Phys. Chem. A 2018, 122(3), 811-821. 
55. Segado, M.; Reguero, M. Mechanism of the Photochemical Process of Singlet Oxygen Production by Phenalenone. Phys. Chem. Chem. Phys. 2011, 13(9), 4138.

56. Flors, C.; Ogilby, P. R.; Luis, J. G.; Grillo, T. A.; Izquierdo, L. R.; Gentili, P.-L.; Bussotti, L.; Nonell, S. Phototoxic Phytoalexins. Processes That Compete with the Photosensitized Production of Singlet Oxygen by 9-Phenylphenalenones. Photochem. Photobiol. 2006, 82(1), 95-103.

57. Casellas, J.; Alcover-Fortuny, G.; de Graaf, C.; Reguero, M. Phenylazopyridine as Switch in Photochemical Reactions. A Detailed Computational Description of the Mechanism of Its Photoisomerization. Mater. (Basel, Switzerland) 2017, 10(12), 1342.

58. Casellas, J.; Bearpark, M. J.; Reguero, M. Excited-State Decay in the Photoisomerisation of Azobenzene: A New Balance between Mechanisms. ChemPhysChem 2016, 17(19), 3068-3079.

59. Dai, X.; Song, D.; Liu, K.; Su, H. Photoinduced C-I Bond Homolysis of 5-Iodouracil: A Singlet Predissociation Pathway. J. Chem. Phys. 2017, 146(2) 025103.

60. Yuan, B.; Bernstein, E. R. Initial Mechanisms for the Unimolecular Decomposition of Electronically Excited Bisfuroxan Based Energetic Materials. J. Chem. Phys. 2017, 146(1), 014301.

61. Yuan, B.; Bernstein, E. R. Initial Mechanisms for the Unimolecular Decomposition of Electronically Excited Nitrogen-Rich Energetic Salts with Tetrazole Rings: (NH4) 2BT and TAGzT. J. Chem. Phys. 2016, 145(6) 064306.

62. Yuan, B.; Yu, Z.; Bernstein, E. R. Initial Mechanisms for the Decomposition of Electronically Excited Energetic Salts: TKX-50 and MAD-X1. J. Phys. Chem. A 2015, 119(12), 2965-2981.

63. Yuan, B.; Yu, Z.; Bernstein, E. R. Azole Energetic Materials: Initial Mechanisms for the Energy Release from Electronical Excited Nitropyrazoles. J. Chem. Phys. 2014, 140(3) 034320.

64. Yuan, B.; Yu, Z.; Bernstein, E. R. Initial Decomposition Mechanism for the Energy Release from Electronically Excited Energetic Materials: FOX-7 (1,1-Diamino-2,2Dinitroethene, C2H4N4O4). J. Chem. Phys. 2014, 140(7), 074708.

65. Yuan, B.; Shin, J.-W.; Bernstein, E. R. Dynamics and Fragmentation of van Der Waals and Hydrogen Bonded Cluster Cations: (NH3)n and (NH3BH3)n Ionized at 10.51 EV. J. Chem. Phys. 2016, 144(14), 144315.

66. Datar, A.; Hazra, A. Pathways for Excited-State Nonradiative Decay of 5,6Dihydroxyindole, a Building Block of Eumelanin. J. Phys. Chem. A 2017, 121(14), 2790-2797.

67. Kobayashi, Y.; Okajima, H.; Sotome, H.; Yanai, T.; Mutoh, K.; Yoneda, Y.; Shigeta, Y.; Sakamoto, A.; Miyasaka, H.; Abe, J. Direct Observation of the Ultrafast Evolution of Open-Shell Biradical in Photochromic Radical Dimer. J. Am. Chem. Soc. 2017, 139(18), 6382-6389.

68. Kim, J.; Moon, J.; Sik Lim, J. Theoretical Investigation of the Reaction Mechanism of the Photoisomerization of 1,2-Dihydro-1,2-Azaborine. ChemPhysChem 2015, 16, $1670-1675$.

69. Gao, A.; Zhang, P.; Zhao, M.; Liu, J. Photoisomerization Mechanism of 1,1'-Dimethyl-2,2'-Pyridocyanine in the Gas Phase and in Solution. Spectrochim. Acta A Mol. Biomol. Spectrosc. 2015, 136(PB), 1157-1166.

70. Lei, Y.; Yu, L.; Zhou, B.; Zhu, C.; Wen, Z.; Lin, S. H. Landscapes of Four-Enantiomer Conical Intersections for Photoisomerization of Stilbene: CASSCF Calculation. J. Phys. Chem. A 2014, 118(39), 9021-9031.

71. Suaud, N.; Ruamps, R.; Malrieu, J. P.; Guihery, N. Singly Occupied MOs in Monoand Diradical Conjugated Hydrocarbons: Comparison between Variational SingleReference, Pi-Fully Correlated and Huckel Descriptions. J. Phys. Chem. A 2014, 118(31), 5876-5884. 
72. Zhang, Y.; Wang, L.; Li, Y.; Zhang, J. Theoretical Insight into Electronic Spectra of Carbon Chain Carbenes H 2Cn ( $\mathrm{n}=3-10) . J$. Chem. Phys. 2013, 138(2013), 204303.

73. Hlavacek, N. C.; McAnally, M. O.; Drucker, S. Lowest Triplet (n, Pi*) Electronic State of Acrolein: Determination of Structural Parameters by Cavity Ringdown Spectroscopy and Quantum-Chemical Methods. J. Chem. Phys. 2013, 138(6), 064303.

74. Wheeler, M. D.; Newman, S. M.; Orr-Ewing, A. J.; Ashfold, M. N. R. Cavity Ring-down Spectroscopy. J. Chem. Soc. Faraday Trans. 1998, 94(3), 337-351.

75. Liu, F.; Morokuma, K. Computational Study on the Working Mechanism of a Stilbene Light-Driven Molecular Rotary Motor: Sloped Minimal Energy Path and Unidirectional Nonadiabatic Photoisomerization. J. Am. Chem. Soc. 2012, 134(10), 4864-4876.

76. Li, Y.; Liu, F.; Wang, B.; Su, Q.; Wang, W.; Morokuma, K. Different Conical Intersections Control Nonadiabatic Photochemistry of Fluorene Light-Driven Molecular Rotary Motor: A CASSCF and Spin-Flip DFT Study. J. Chem. Phys. 2016, 145(24), 244311.

77. Ben Amor, N.; Soupart, A.; Heitz, M. C. Methodological CASPT2 Study of the Valence Excited States of an Iron-Porphyrin Complex. J. Mol. Model. 2017, $23(2), 53$.

78. Venturinelli Jannuzzi, S. A.; Phung, Q. M.; Domingo, A.; Formiga, A. L. B.; Pierloot, K. Spin State Energetics and Oxyl Character of Mn-Oxo Porphyrins by Multiconfigurational Ab Initio Calculations: Implications on Reactivity. Inorg. Chem. 2016, 55(11), 5168-5179.

79. Bai, F. Q.; Nakatani, N.; Nakayama, A.; Hasegawa, J. Y. Excited States of a Significantly Ruffled Porphyrin: Computational Study on Structure-Induced Rapid Decay Mechanism via Intersystem Crossing. J. Phys. Chem. A 2014, 118(23), 4184-4194.

80. Nenov, A.; Giussani, A.; Segarra-Martí, J.; Jaiswal, V. K.; Rivalta, I.; Cerullo, G.; Mukamel, S.; Garavelli, M. Modeling the High-Energy Electronic State Manifold of Adenine: Calibration for Nonlinear Electronic Spectroscopy. J. Chem. Phys. 2015, 142(21), 0-17.

81. Segarra-Martí, J.; Garavelli, M.; Aquilante, F. Multiconfigurational Second-Order Perturbation Theory with Frozen Natural Orbitals Extended to the Treatment of Photochemical Problems. J. Chem. Theory Comput. 2015, 11(8), 3772-3784.

82. Bricker, W. P.; Lo, C. S. Efficient Pathways of Excitation Energy Transfer from Delocalized $\mathrm{S}_{2}$ Excitons in the Peridinin-Chlorophyll a-Protein Complex. J. Phys. Chem. B 2015, 119(18), 5755-5764.

83. Bricker, W. P.; Lo, C. S. Excitation Energy Transfer in the Peridinin-Chlorophyll a-Protein Complex Modeled Using Configuration Interaction. J. Phys. Chem. B 2014, 118(31), 9141-9154.

84. Guan, P. J.; Cui, G.; Fang, Q. Computational Photochemistry of the Azobenzene Scaffold of Sudan I and Orange II Dyes: Excited-State Proton Transfer and Deactivation via Conical Intersections. ChemPhys Chem 2015, 16(4), 805-811.

85. Francés-Monerris, A.; Segarra-Martí, J.; Merchán, M.; Roca-Sanjuán, D. CompleteActive-Space Second-Order Perturbation Theory (CASPT2//CASSCF) Study of the Dissociative Electron Attachment in Canonical DNA Nucleobases Caused by Low-Energy Electrons (0-3 EV). J. Chem. Phys. 2015, 143(21), 0-14.

86. Giussani, A.; Segarra-Martí, J.; Nenov, A.; Rivalta, I.; Tolomelli, A.; Mukamel, S.; Garavelli, M. Spectroscopic Fingerprints of DNA/RNA Pyrimidine Nucleobases in Third-Order Nonlinear Electronic Spectra. Theor. Chem. Acc. 2016, 135(5), 1-18.

87. Segarra-Martí, J.; Francés-Monerris, A.; Roca-Sanjuán, D.; Merchán, M. Assessment of the Potential Energy Hypersurfaces in Thymine within Multiconfigurational Theory: CASSCF vs. CASPT2. Molecules 2016, 21(12), 1-18.

88. Santolini, V.; Malhado, J. P.; Robb, M. A.; Garavelli, M.; Bearpark, M. J. Photochemical Reaction Paths of Cis-Dienes Studied with RASSCF: The Changing Balance Between Ionic and Covalent Excited States. Mol. Phys. 2015, 113(13-14), 1978-1990. 
89. Xia, S. H.; Liu, X. Y.; Fang, Q.; Cui, G. Excited-State Ring-Opening Mechanism of Cyclic Ketones: A MS-CASPT2//CASSCF Study. J. Phys. Chem. A 2015, 119(15), 3569-3576.

90. Bera, A.; Bhattacharya, A. Excited Electronic State Decomposition Mechanisms of Clusters of Dimethylnitramine and Aluminum. J. Chem. Sci. 2015, 127(1), 71-82.

91. Bera, A.; Maroo, S.; Bhattacharya, A. Electronically Nonadiabatic Decomposition Mechanisms of Clusters of Zinc and Dimethylnitramine. Chem. Phys. 2015, 446, 47-56.

92. Bera, A.; Ghosh, J.; Bhattacharya, A. Ab Initio Multiple Spawning Dynamics Study of Dimethylnitramine and Dimethylnitramine-Fe Complex to Model Their Ultrafast Nonadiabatic Chemistry. J. Chem. Phys. 2017, 147(4), 044308.

93. Manni, G. L.; Smart, S. D.; Alavi, A. Combining the Complete Active Space Self-Consistent Field Method and the Full Configuration Interaction Quantum Monte Carlo within a Super-CI Framework, With Application to Challenging MetalPorphyrins. J. Chem. Theory Comput. 2016, 12(3), 1245-1258.

94. Asturiol, D.; Barbatti, M. Electronic States of Porphycene- $\mathrm{O}_{2}$ Complex and Photoinduced Singlet $\mathrm{O}_{2}$ Production. J. Chem. Phys. 2013, 139(7), 074307.

95. Kornobis, K.; Kumar, N.; Lodowski, P.; Jaworska, M.; Piecuch, P.; Lutz, J. J.; Wong, B. M.; Kozlowski, P. M. Electronic Structure of the S1 State in Methylcobalamin: Insight from CASSCF/MC-XQDPT2, EOM-CCSD, and TD-DFT Calculations. J. Comput. Chem. 2013, 34(12), 987-1004.

96. Stich, T. A.; Brooks, A. J.; Buan, N. R.; Brunold, T. C. Spectroscopic and Computational Studies of Co3+-Corrinoids: Spectral and Electronic Properties of the B12 Cofactors and Biologically Relevant Precursors. J. Am. Chem. Soc. 2003, 125(19), $5897-5914$.

97. Brunold, T. C.; Conrad, K. S.; Liptak, M. D.; Park, K. Spectroscopically Validated Density Functional Theory Studies of the B12 Cofactors and Their Interactions With Enzyme Active Sites. Coord. Chem. Rev. 2009, 253(5-6), 779-794.

98. Walker, L. A.; Jarrett, J. T.; Anderson, N. A.; Pullen, S. H.; Matthews, R. G.; Sension, R. J. Time-Resolved Spectroscopic Studies of B12 Coenzymes: The Identification of a Metastable Cob(III)Alamin Photoproduct in the Photolysis of Methylcobalamin. J. Am. Chem. Soc. 1998, 120(15), 3597-3603.

99. Shiang, J. J.; Walker, L. A.; Anderson, N. A.; Cole, A. G.; Sension, R. J. TimeResolved Spectroscopic Studies of B 12 Coenzymes: The Photolysis of Methylcobalamin Is Wavelength Dependent. J. Phys. Chem. B 1999, 103(47), 10532-10539.

100. Harris, D. A.; Stickrath, A. B.; Carroll, E. C.; Sension, R. J. Influence of Environment on the Electronic Structure of Cob(III)Alamins: Time-Resolved Absorption Studies of the S1 State Spectrum and Dynamics. J. Am. Chem. Soc. 2007, 129(24), 7578-7585.

101. Huix-Rotllant, M.; Siri, D.; Ferré, N. Theoretical Study of the Photochemical Generation of Triplet Acetophenone. Phys. Chem. Chem. Phys. 2013, 15(44), 19293-19300.

102. Xu, X.; Yang, K. R.; Truhlar, D. G. Diabatic Molecular Orbitals, Potential Energies, and Potential Energy Surface Couplings by the 4-Fold Way for Photodissociation of Phenol. J. Chem. Theory Comput. 2013, 9(8), 3612-3625.

103. Kletskii, M. E.; Lisovin, A. V.; Burov, O. N.; Kurbatov, S. V. Competing Mechanisms of Norrish and Norrish-like Reactions in a Wide Range of Systems-From Carbonyl Compounds to Nitrogen Oxide Donators. Comput. Theor. Chem. 2014, 1047(1), $55-66$.

104. Grabarek, D.; Walczak, E.; Andruniow, T. Assessing the Accuracy of Various Ab Initio Methods for Geometries and Excitation Energies of Retinal Chromophore Minimal Model by Comparison with CASPT3 Results. J. Chem. Theory Comput. 2016, 12(5), 2346-2356. 
105. Walczak, E.; Szefczyk, B.; Andruniów, T. Geometries and Vertical Excitation Energies in Retinal Analogues Resolved at the CASPT2 Level of Theory: Critical Assessment of the Performance of CASSCF, CC2, and DFT Methods. J. Chem. Theory Comput. 2013, 9(11), 4915-4927.

106. Szefczyk, B.; Grabarek, D.; Walczak, E.; Andruniów, T. Excited-State Minima and Emission Energies of Retinal Chromophore Analogues: Performance of CASSCF and CC2 Methods as Compared With CASPT2. J. Comput. Chem. 2017, 38(20), 1799-1810.

107. Gozem, S.; Huntress, M.; Schapiro, I.; Lindh, R.; Granovsky, A. A.; Angeli, C.; Olivucci, M. Dynamic Electron Correlation Effects on the Ground State Potential Energy Surface of a Retinal Chromophore Model. J. Chem. Theory Comput. 2012, 8(11), 4069-4080.

108. Huix-Rotllant, M.; Filatov, M.; Gozem, S.; Schapiro, I.; Olivucci, M.; Ferré, N. Assessment of Density Functional Theory for Describing the Correlation Effects on the Ground and Excited State Potential Energy Surfaces of a Retinal Chromophore Model. J. Chem. Theory Comput. 2013, 9(9), 3917-3932.

109. Xu, X.; Gozem, S.; Olivucci, M.; Truhlar, D. G. Combined Self-Consistent-Field and Spin-Flip Tamm-Dancoff Density Functional Approach to Potential Energy Surfaces for Photochemistry. J. Phys. Chem. Lett. 2013, 4(2), 253-258.

110. Gozem, S.; Krylov, A. I.; Olivucci, M. Conical Intersection and Potential Energy Surface Features of a Model Retinal Chromophore: Comparison of EOM-CC and Multireference Methods. J. Chem. Theory Comput. 2013, 9(1), 284-292.

111. Gozem, S.; Melaccio, F.; Lindh, R.; Krylov, A. I.; Granovsky, A. A.; Angeli, C.; Olivucci, M. Mapping the Excited State Potential Energy Surface of a Retinal Chromophore Model with Multireference and Equation-of-Motion Coupled-Cluster Methods. J. Chem. Theory Comput. 2013, 9(10), 4495-4506.

112. Gozem, S.; Melaccio, F.; Valentini, A.; Filatov, M.; Huix-Rotllant, M.; Ferre, N.; Frutos, L. M.; Angeli, C.; Krylov, A. I.; Granovsky, A. A.; et al. Shape of Multireference, Equation-of-Motion Coupled-Cluster, and Density Functional Theory Potential Energy Surfaces at a Conical Intersection. J. Chem. Theory Comput. 2014, 10(8), 3074-3084.

113. Zen, A.; Coccia, E.; Gozem, S.; Olivucci, M.; Guidoni, L. Quantum Monte Carlo Treatment of the Charge Transfer and Diradical Electronic Character in a Retinal Chromophore Minimal Model. J. Chem. Theory Comput. 2015, 11(3), 992-1005.

114. Tuna, D.; Lefrancois, D.; Wolanski, L.; Gozem, S.; Schapiro, I.; Andruniow, T.; Dreuw, A.; Olivucci, M. Assessment of Approximate Coupled-Cluster and Algebraic-Diagrammatic-Construction Methods for Ground- and Excited-State Reaction Paths and the Conical-Intersection Seam of a Retinal-Chromophore Model. J. Chem. Theory Comput. 2015, 11(12), 5758-5781.

115. Chin, C.-H.; Lin, S. H. Theoretical Investigations of Absorption and Fluorescence Spectra of Protonated Pyrene. Phys. Chem. Chem. Phys. 2016, 18(21), 14569-14579.

116. Cacelli, I.; Ferretti, A.; Prampolini, G. Predicting Light Absorption Properties of Anthocyanidins in Solution: A Multi-Level Computational Approach. Theor. Chem. Acc. 2016, 135(6), 1-17.

117. Fréneau, M.; de Sainte Claire, P.; Hoffmann, N.; Vors, J.-P.; Geist, J.; Euvrard, M.; Richard, C. Phototransformation of Tetrazoline Oxime Ethers: Photoisomerization vs Photodegradation. RSC Adv. 2016, 6(7), 5512-5522.

118. Fréneau, M.; Hoffmann, N.; Vors, J.-P.; Genix, P.; Richard, C.; De Sainte Claire, P. Phototransformation of Tetrazoline Oxime Ethers-Part 2: Theoretical Investigation. RSC Adv. 2016, 6(68), 63965-63972. 
119. López-de-Luzuriaga, J. M.; Manso, E.; Monge, M.; Sampedro, D. Dual Fluorescence of 4-(Dimethylamino)-Pyridine: A Comparative Linear Response TDDFT versus State-Specific CASSCF Study Including Solvent With the PCM Model. Theor. Chem. Acc. 2015, 134(5), 55.

120. Li, J.; Rinkevicius, Z.; Cao, Z. A Time-Dependent Density-Functional Theory and Complete Active Space Self-Consistent Field Method Study of Vibronic Absorption and Emission Spectra of Coumarin. J. Chem. Phys. 2014, 141(1), 014306.

121. Olsen, S. Locally-Excited (LE) versus Charge-Transfer (CT) Excited State Competition in a Series of Para-Substituted Neutral Green Fluorescent Protein (GFP) Chromophore Models. J. Phys. Chem. B 2015, 119(6), 2566-2575.

122. Ai, Y.; Tian, G.; Luo, Y. Role of Non-Condon Vibronic Coupling and Conformation Change on Two-Photon Absorption Spectra of Green Fluorescent Protein. Mol. Phys. 2013, 111(9-11), 1316-1321.

123. Zhang, L.; Muchall, H. M.; Peslherbe, G. H. Substituent Effects in the Absorption Spectra of Phenol Radical Species: Origin of the Redshift Caused by 3,5-Dimethoxyl Substitution. Photochem. Photobiol. 2013, 89(3), 536-544.

124. Palmer, M. H.; Hoffmann, S. V.; Jones, N. C.; Coreno, M.; de Simone, M.; Grazioli, C.; Peterson, K. A.; Baiardi, A.; Zhang, T.; Biczysko, M. A Combined Theoretical and Experimental Study of the Valence and Rydberg States of Iodopentafluorobenzene. J. Chem. Phys. 2017, 146(17), 174301.

125. Palmer, M. H.; Coreno, M.; de Simone, M.; Hoffmann, S. V.; Jones, N. C.; Grazioli, C.; Peterson, K. A.; Baiardi, A.; Zhang, T.; Biczysko, M. A Combined Theoretical and Experimental Study of the Ionic States of Iodopentafluorobenzene. J. Chem. Phys. 2017, 146(8) 084302.

126. Palmer, M. H.; Biczysko, M.; Baiardi, A.; Coreno, M.; De Simone, M.; Grazioli, C.; Hoffmann, S. V.; Jones, N. C.; Peterson, K. A. The Ionic States of Difluoromethane: A Reappraisal of the Low Energy Photoelectron Spectrum Including Ab Initio Configuration Interaction Computations. J. Chem. Phys. 2017, 147(7), 074305.

127. Palmer, M. H.; Hoffmann, S. V.; Jones, N. C.; Smith, E. R.; Lichtenberger, D. L. The Electronic States of Pyridine-N-Oxide Studied by VUV Photoabsorption and $\mathrm{Ab}$ Initio Configuration Interaction Computations. J. Chem. Phys. 2013, 138(21), 214317.

128. Palmer, M. H.; Ridley, T.; Hoffmann, S. V.; Jones, N. C.; Coreno, M.; De Simone, M.; Grazioli, C.; Biczysko, M.; Baiardi, A.; Limão-Vieira, P. Interpretation of the Vacuum Ultraviolet Photoabsorption Spectrum of Iodobenzene by Ab Initio Computations. J. Chem. Phys. 2015, 142(13), 134302.

129. Palmer, M. H.; Ridley, T.; Vrønning Hoffmann, S.; Jones, N. C.; Coreno, M.; De Simone, M.; Grazioli, C.; Zhang, T.; Biczysko, M.; Baiardi, A.; et al. Combined Theoretical and Experimental Study of the Valence, Rydberg, and Ionic States of Chlorobenzene. J. Chem. Phys. 2016, 144(12), 124302.

130. Besley, N. A. Calculation of the Electronic Spectra of Molecules in Solution and on Surfaces. Chem. Phys. Lett. 2004, 390(1-3), 124-129.

131. Jacquemin, D.; Perpe, E. A.; Scuseria, G. E.; Ciofini, I.; Adamo, C. TD-DFT Performance for the Visible Absorption Spectra of Organic Dyes: Conventional versus LongRange Hybrids. J. Chem. Theory Comput. 2008, 4, 123-135.

132. Jacquemin, D.; Perpète, E. A.; Ciofini, I.; Adamo, C. Assessment of the $\Omega B 97$ Family for Excited-State Calculations. Theor. Chem. Acc. 2011, 128(1), 127-136.

133. Goerigk, L.; Grimme, S. Assessment of TD-DFT Methods and of Various Spin Scaled CIS(D) and CC2 Versions for the Treatment of Low-Lying Valence Excitations of Large Organic Dyes. J. Chem. Phys. 2010, 132(18) 184103. 
134. Rohrdanz, M. A.; Martins, K. M.; Herbert, J. M. A Long-Range-Corrected Density Functional That Performs Well for Both Ground-State Properties and TimeDependent Density Functional Theory Excitation Energies, Including ChargeTransfer Excited States. J. Chem. Phys. 2009, 130(5), 054112.

135. Vydrov, O. A.; Heyd, J.; Krukau, A. V.; Scuseria, G. E. Importance of Short-Range versus Long-Range Hartree-Fock Exchange for the Performance of Hybrid Density Functionals. J. Chem. Phys. 2006, 125(7), 0-9.

136. Peach, M. J. G.; Helgaker, T.; Sałek, P.; Keal, T. W.; Lutnæs, O. B.; Tozer, D. J.; Handy, N. C. Assessment of a Coulomb-Attenuated Exchange-Correlation Energy Functional. Phys. Chem. Chem. Phys. 2006, 8(5), 558-562.

137. Peach, M. J. G.; Cohen, A. J.; Tozer, D. J. Influence of Coulomb-Attenuation on Exchange-Correlation Functional Quality. Phys. Chem. Chem. Phys. 2006, 8(39), 4543-4549.

138. Rohrdanz, M. A.; Herbert, J. M. Simultaneous Benchmarking of Ground- and Excited-State Properties with Long-Range-Corrected Density Functional Theory. J. Chem. Phys. 2008, 129(3), 034107.

139. Shcherbin, D.; Ruud, K. The Use of Coulomb-Attenuated Methods for the Calculation of Electronic Circular Dichroism Spectra. Chem. Phys. 2008, 349(1-3), 234-243.

140. Kuritz, N.; Stein, T.; Baer, R.; Kronik, L. Charge-Transfer-like $\Pi \rightarrow \pi^{*}$ Excitations in Time-Dependent Density Functional Theory: A Conundrum and Its Solution. J. Chem. Theory Comput. 2011, 7(8), 2408-2415.

141. Stein, T.; Kronik, L.; Baer, R. Reliable Prediction of Charge Transfer Excitations in Molecular Complexesusing Time-Dependent Density Functional Theory. J. Am. Chem. Soc. 2009, 131(8), 2818-2820.

142. Stein, T.; Eisenberg, H.; Kronik, L.; Baer, R. Fundamental Gaps in Finite Systems from Eigenvalues of a Generalized Kohn-Sham Method. Phys. Rev. Lett. 2010, 105(26), 1-4.

143. Stein, T.; Kronik, L.; Baer, R. Prediction of Charge-Transfer Excitations in Coumarin-Based Dyes Using a Range-Separated Functional Tuned From First Principles. J. Chem. Phys. 2009, 131(24), 244119.

144. Gąsiorski, P.; Matusiewicz, M.; Gondek, E.; Uchacz, T.; Wojtasik, K.; Danel, A.; Shchur, Y.; Kityk, A. V. On the Spectral Properties of Methyl and Methoxy Derivatives of 1,3-Diphenyl-1H-Pyrazolo[3,4-b]Quinoxalines: Experiment and DFT/ TDDFT Calculations. Spectrochim. Acta A Mol. Biomol. Spectrosc. 2017, 186, 89-98.

145. Gąsiorski, P.; Matusiewicz, M.; Gondek, E.; Pokladko-Kowar, M.; Armatys, P.; Wojtasik, K.; Danel, A.; Uchacz, T.; Kityk, A. V. Efficient Green Electroluminescence from 1,3-Diphenyl-1H-Pyrazolo[3,4-b]Quinoxaline Dyes in Dye-Doped Polymer Based Electroluminescent Devices. Dye. Pigment. 2018, 151(January), 380-384.

146. Arulmozhiraja, S.; Coote, M. L. 1La and 1Lb States of Indole and Azaindole: Is Density Functional Theory Inadequate?J. Chem. Theory Comput. 2012, 8(2), 575-584.

147. Eriksen, J. J.; Sauer, S. P. A.; Mikkelsen, K. V.; Christiansen, O.; Jensen, H. J. A.; Kongsted, J. Failures of TDDFT in Describing the Lowest Intramolecular ChargeTransfer Excitation in Para-Nitroaniline. Mol. Phys. 2013, 111(9-11), 1235-1248.

148. Körzdörfer, T.; Brédas, J. L. Organic Electronic Materials: Recent Advances in the DFT Description of the Ground and Excited States Using Tuned Range-Separated Hybrid Functionals. Acc. Chem. Res. 2014, 47(11), 3284-3291.

149. Sun, H.; Zhong, C.; Brédas, J. L. Reliable Prediction With Tuned Range-Separated Functionals of the Singlet-Triplet Gap in Organic Emitters for Thermally Activated Delayed Fluorescence. J. Chem. Theory Comput. 2015, 11(8), 3851-3858.

150. Penfold, T. J. On Predicting the Excited-State Properties of Thermally Activated Delayed Fluorescence Emitters. J. Phys. Chem. C 2015, 119(24), 13535-13544. 
151. Moore, B.; Autschbach, J. Longest-Wavelength Electronic Excitations of Linear Cyanines: The Role of Electron Delocalization and of Approximations in TimeDependent Density Functional Theory. J. Chem. Theory Comput. 2013, 9(11), 4991-5003.

152. Maschietto, F.; Campetella, M.; Frisch, M. J.; Scalmani, G.; Adamo, C.; Ciofini, I. How Are the Charge Transfer Descriptors Affected by the Quality of the Underpinning Electronic Density?J. Comput. Chem. 2018, 735-742.

153. Shu, Y.; Parker, K. A.; Truhlar, D. G. Dual-Functional Tamm-Dancoff Approximation: A Convenient Density Functional Method That Correctly Describes S1/S0Conical Intersections. J. Phys. Chem. Lett. 2017, 8(10), 2107-2112.

154. Grimme, S. A Simplified Tamm-Dancoff Density Functional Approach for the Electronic Excitation Spectra of Very Large Molecules. J. Chem. Phys. 2013, 138(24), 0-14.

155. Bannwarth, C.; Grimme, S. A Simplified Time-Dependent Density Functional Theory Approach for Electronic Ultraviolet and Circular Dichroism Spectra of Very Large Molecules. Comput. Theor. Chem. 2014, 1040-1041, 45-53.

156. Risthaus, T.; Hansen, A.; Grimme, S. Excited States Using the Simplified TammDancoff-Approach for Range-Separated Hybrid Density Functionals: Development and Application. Phys. Chem. Chem. Phys. 2014, 16(28), 14408-14419.

157. Bannwarth, C.; Seibert, J.; Grimme, S. Electronic Circular Dichroism of [16]Helicene With Simplified TD-DFT: Beyond the Single Structure Approach. Chirality 2016, 28, 365-369.

158. Grimme, S.; Bannwarth, C. Ultra-Fast Computation of Electronic Spectra for Large Systems by Tight-Binding Based Simplified Tamm-Dancoff Approximation (STDA-XTB). J. Chem. Phys. 2016, 145(5), 054103.

159. Seibert, J.; Bannwarth, C.; Grimme, S. Biomolecular Structure Information from High-Speed Quantum Mechanical Electronic Spectra Calculation. J. Am. Chem. Soc. 2017, 139(34), 11682-11685.

160. Bannwarth, C.; Grimme, S. Electronic Circular Dichroism of Highly Conjugated $\pi$-Systems: Breakdown of the Tamm-Dancoff/Configuration Interaction Singles Approximation. J. Phys. Chem. A 2015, 119(15), 3653-3662.

161. Zvereva, E. E.; Grimme, S.; Katsyuba, S. A.; Burganov, T. I.; Zagidullin, A. A.; Milyukov, V. A.; Sinyashin, O. G. Application of Time-Dependent Density Functional Theory and Optical Spectroscopy toward the Rational Design of Novel 3,4,5-Triaryl-1-R-1,2-Diphospholes. J. Phys. Chem. A 2013, 117(31), 6827-6834.

162. Burganov, T. I.; Zhukova, N. A.; Mamedov, V. A.; Bannwarth, C.; Grimme, S.; Katsyuba, S. A. Benzimidazolylquinoxalines: Novel Fluorophores with Tuneable Sensitivity to Solvent Effects. Phys. Chem. Chem. Phys. 2017, 19(8), 6095-6104.

163. Jacquemin, D.; Duchemin, I.; Blase, X. 0-0 Energies Using Hybrid Schemes: Benchmarks of TD-DFT, CIS(D), ADC(2), CC2, and BSE/GW Formalisms for 80 Real-Life Compounds. J. Chem. Theory Comput. 2015, 11(11), 5340-5359.

164. Ameline, D.; Diring, S.; Farre, Y.; Pellegrin, Y.; Naponiello, G.; Blart, E.; Charrier, B.; Dini, D.; Jacquemin, D.; Odobel, F. Isoindigo Derivatives for Application in P-Type Dye Sensitized Solar Cells. RSC Adv. 2015, 5(104), 85530-85539.

165. Houari, Y.; Charaf-Eddin, A.; Laurent, A. D.; Massue, J.; Ziessel, R.; Ulrich, G.; Jacquemin, D. Modeling Optical Signatures and Excited-State Reactivities of Substituted Hydroxyphenylbenzoxazole (HBO) ESIPT Dyes. Phys. Chem. Chem. Phys. 2014, 16(4), 1319-1321.

166. Ji, S.; Ge, J.; Escudero, D.; Wang, Z.; Zhao, J.; Jacquemin, D. Molecular StructureIntersystem Crossing Relationship of Heavy-Atom-Free Bodipy Triplet Photosensitizers. J. Org. Chem. 2015, 80(11), 5958-5963. 
167. Fihey, A.; Perrier, A.; Browne, W. R.; Jacquemin, D. Multiphotochromic Molecular Systems. Chem. Soc. Rev. 2015, 3719-3759.

168. Chibani, S.; Charaf-Eddin, A.; Mennucci, B.; Le Guennic, B.; Jacquemin, D. Optical Signatures of OBO Fluorophores: A Theoretical Analysis. J. Chem. Theory Comput. 2014, 10(2), 805-815.

169. Jacquemin, D.; Moore, B.; Adamo, C.; Autschbach, J. Performance of an Optimally Tuned Range-Separated Hybrid Functional for 0-0 Electronic Excitation Energies. J. Chem. Theory Comput. 2014, 10, 1677-1685.

170. Farré, Y.; Zhang, L.; Pellegrin, Y.; Planchat, A.; Blart, E.; Boujtita, M.; Hammarström, L.; Jacquemin, D.; Odobel, F. Second Generation of Diketopyrrolopyrrole Dyes for NiO-Based Dye-Sensitized Solar Cells. J. Phys. Chem. C 2016, 120(15), 7923-7940.

171. Boixel, J.; Guerchais, V.; Le Bozec, H.; Jacquemin, D.; Amar, A.; Boucekkine, A.; Colombo, A.; Dragonetti, C.; Marinotto, D.; Roberto, D.; et al. Second-Order NLO Switches from Molecules to Polymer Films Based on Photochromic Cyclometalated Platinum(II) Complexes. J. Am. Chem. Soc. 2014, 136(14), $5367-5375$.

172. Le Guennic, B.; Jacquemin, D. Taking Up the Cyanine Challenge with Quantum Tools. Acc. Chem. Res. 2015, 48(3), 530-537.

173. Jacquemin, D.; Duchemin, I.; Blase, X. Benchmarking the Bethe-Salpeter Formalism on a Standard Organic Molecular Set. J. Chem. Theory Comput. 2015, 11(7), 3290-3304.

174. Houari, Y.; Chibani, S.; Jacquemin, D.; Laurent, A. D. TD-DFT Assessment of the Excited State Intramolecular Proton Transfer in Hydroxyphenylbenzimidazole (HBI) Dyes. J. Phys. Chem. B 2015, 119, 2180-2192.

175. Azarias, C.; Budzák, Š.; Laurent, A. D.; Ulrich, G.; Jacquemin, D. Tuning ESIPT Fluorophores into Dual Emitters. Chem. Sci. 2016, 7(6), 3763-3774.

176. Benelhadj, K.; Muzuzu, W.; Massue, J.; Retailleau, P.; Charaf-Eddin, A.; Laurent, A. D.; Jacquemin, D.; Ulrich, G.; Ziessel, R. White Emitters by Tuning the Excited-State Intramolecular Proton-Transfer Fluorescence Emission in 2-(2-Hydroxybenzofuran)Benzoxazole Dyes. Chem. A Eur. J. 2014, 20(40), 12843-12857.

177. Kamada, K.; Namikawa, T.; Senatore, S.; Matthews, C.; Lenne, P. F.; Maury, O.; Andraud, C.; Ponce-Vargas, M.; Le Guennic, B.; Jacquemin, D.; et al. Boron Difluoride Curcuminoid Fluorophores With Enhanced Two-Photon Excited Fluorescence Emission and Versatile Living-Cell Imaging Properties. Chem. A Eur. J. 2016, 22(15), 5219-5232.

178. Escudero, D.; Jacquemin, D. Computational Insights Into the Photodeactivation Dynamics of Phosphors for OLEDs: A Perspective. Dalton Trans. 2015, 44(18), 8346-8355.

179. Laurent, A. D.; Adamo, C.; Jacquemin, D. Dye Chemistry With Time-Dependent Density Functional Theory. Phys. Chem. Chem. Phys. 2014, 16(28), 14334-14356.

180. Guido, C. A.; Jacquemin, D.; Adamo, C.; Mennucci, B. Electronic Excitations in Solution: The Interplay between State Specific Approaches and a Time-Dependent Density Functional Theory Description. J. Chem. Theory Comput. 2015, 11(12), 5782-5790.

181. Charaf-Eddin, A.; Le Guennic, B.; Jacquemin, D. Excited-States of BODIPYCyanines: Ultimate TD-DFT Challenges?RSC Adv. 2014, 4(90), 49449-49456.

182. Pascal, S.; Haefele, A.; Monnereau, C.; Charaf-Eddin, A.; Jacquemin, D.; Le Guennic, B.; Andraud, C.; Maury, O. Expanding the Polymethine Paradigm: Evidence for the Contribution of a Bis-Dipolar Electronic Structure. J. Phys. Chem. A 2014, 118(23), 4038-4047. 
183. Boulanger, P.; Jacquemin, D.; Duchemin, I.; Blase, X. Fast and Accurate Electronic Excitations in Cyanines with the Many-Body Bethe-Salpeter Approach. J. Chem. Theory Comput. 2014, 10(3), 1212-1218.

184. Heyer, E.; Benelhadj, K.; Budzák, S.; Jacquemin, D.; Massue, J.; Ulrich, G. On the Fine-Tuning of the Excited-State Intramolecular Proton Transfer (ESIPT) Process in 2-(2'-Hydroxybenzofuran)Benzazole (HBBX) Dyes. Chem. A Eur. J. 2017, 23(30), 7324-7336.

185. Benelhadj, K.; Massue, J.; Retailleau, P.; Chibani, S.; Le Guennic, B.; Jacquemin, D.; Ziessel, R.; Ulrich, G. Solution- and Solid-State Luminescent Borate Complexes Based on a Substituted $\pi$-Conjugated 2-(6 J-Hydroxy-5 J-Benzofuryl) Scaffold. Eur. J. Org. Chem. 2014, 2014(32), 7156-7164.

186. Massue, J.; Benelhadj, K.; Chibani, S.; Le Guennic, B.; Jacquemin, D.; Retailleau, P.; Ulrich, G.; Ziessel, R. Fluorescent 2-(2'-Hydroxybenzofuran)Benzoxazole (HBBO) Borate Complexes: Synthesis, Optical Properties, and Theoretical Calculations. Tetrahedron Lett. 2014, 55(30), 4136-4140.

187. Le Bahers, T.; Pauporté, T.; Lainé, P. P.; Labat, F.; Adamo, C.; Ciofini, I. Modeling Dye-Sensitized Solar Cells: From Theory to Experiment. J. Phys. Chem. Lett. 2013, 4(6), 1044-1050.

188. Mathew, S.; Yella, A.; Gao, P.; Humphry-Baker, R.; Curchod, B. F. E.; AshariAstani, N.; Tavernelli, I.; Rothlisberger, U.; Nazeeruddin, M. K.; Grätzel, M. Dye-Sensitized Solar Cells With 13\% Efficiency Achieved Through the Molecular Engineering of Porphyrin Sensitizers. Nat. Chem. 2014, 6(3), 242-247.

189. Liu, Z.; Hua, S.; Yan, X. Linear and Nonlinear Optical Properties of TriphenylamineIndandione Chromophores: Theoretical Study of the Structure-Function Relationship Under the Combined Action of Substituent and Symmetry Change. J. Phys. Chem. A 2018, 122(9), 2344-2352.

190. Linares-Flores, C.; Schott, E.; Claveria-Cadiz, F.; Zarate, X. Energy Conversion Process of Substituted Phthalocyanines With Potential Application to DSSC: A Theoretical Study. Theor. Chem. Acc. 2018, 137(4), 52.

191. Zarate, X.; Saavedra-Torres, M.; Rodriguez-Serrano, A.; Gomez, T.; Schott, E. Exploring the Relevance of Thiophene Rings as Bridge Unit in Acceptor-BridgeDonor Dyes on Self-Aggregation and Performance in DSSCs. J. Comput. Chem. 2017, 685-698.

192. Zarate, X.; Schott-Verdugo, S.; Rodriguez-Serrano, A.; Schott, E. The Nature of the Donor Motif in Acceptor-Bridge-Donor Dyes as an Influence in the Electron Photo-Injection Mechanism in DSSCs. J. Phys. Chem. A 2016, 120(9), $1613-1624$.

193. Li, Y.; Xu, B.; Song, P.; Ma, F.; Sun, M. D-A-л-A System: Light Harvesting, Charge Transfer, and Molecular Designing. J. Phys. Chem. C 2017, 121(23), 12546-12561.

194. Nachimuthu, S.; Chen, W.-C.; Leggesse, E. G.; Jiang, J.-C. First Principles Study of Organic Sensitizers for Dye Sensitized Solar Cells: Effects of Anchoring Groups on Optoelectronic Properties and Dye Aggregation. Phys. Chem. Chem. Phys. 2016, 18(2), 1071-1081.

195. Baheti, A.; Justin Thomas, K. R.; Lee, C.-P.; Li, C.-T.; Ho, K.-C. Organic Dyes Containing Fluoren-9-Ylidene Chromophores for Efficient Dye-Sensitized Solar Cells. J. Mater. Chem. A 2014, 2(16), 5766.

196. Baheti, A.; Justin Thomas, K. R.; Li, C. T.; Lee, C. P.; Ho, K. C. Fluorene-Based Sensitizers with a Phenothiazine Donor: Effect of Mode of Donor Tethering on the Performance of Dye-Sensitized Solar Cells. ACS Appl. Mater. Interfaces 2015, 7(4), 2249-2262. 
197. Venkateswararao, A.; Thomas, K. R. J.; Lee, C. P.; Li, C. T.; Ho, K. C. Organic Dyes Containing Carbazole as Donor and $\pi$-Linker: Optical, Electrochemical, and Photovoltaic Properties. ACS Appl. Mater. Interfaces 2014, 6(4), 2528-2539.

198. Feng, J.; Jiao, Y.; Ma, W.; Nazeeruddin, M. K.; Grätzel, M.; Meng, S. First Principles Design of Dye Molecules With Ullazine Donor for Dye Sensitized Solar Cells. J. Phys. Chem. C 2013, 117(8), 3772-3778.

199. Kulinich, A. V.; Ishchenko, A. A.; Kukhta, I. N.; Mitryukhin, L. K.; Kazakov, S. M.; Kukhta, A. V. Electron Impact Excitation of the Merocyanine Molecule in the Gas Phase. Chem. Phys. 2018, 503, 20-24.

200. Kowalska-Baron, A.; Zurawinski, R.; Lukasik, B.; Chworos, A.; Wysocki, S. Theoretical and Experimental Study on the Electronic Structure of DistyrylnaphthaleneBased Conjugated Oligoelectrolytes. J. Lumin. 2018, 196(December 2017), 81-89.

201. Lee, S.; Kim, S. O.; Shin, H.; Yun, H. J.; Yang, K.; Kwon, S. K.; Kim, J. J.; Kim, Y. H. Deep-Blue Phosphorescence from Perfluoro Carbonyl-Substituted Iridium Complexes. J. Am. Chem. Soc. 2013, 135(38), 14321-14328.

202. Estrada, L. A.; Stalder, R.; Abboud, K. A.; Risko, C.; Brédas, J. L.; Reynolds, J. R. Understanding the Electronic Structure of Isoindigo in Conjugated Systems: A Combined Theoretical and Experimental Approach. Macromolecules 2013, 46(22), 8832-8844.

203. Lourenço Neto, M.; Agra, K. L.; Suassuna Filho, J.; Jorge, F. E. TDDFT Calculations and Photoacoustic Spectroscopy Experiments Used to Identify Phenolic Acid Functional Biomolecules in Brazilian Tropical Fruits in Natura. Spectrochim. Acta A Mol. Biomol. Spectrosc. 2018, 193, 249-257.

204. Ma, H.; Huang, J.-D. Ab Initio Study of the Excited-State Proton Transfer Mechanisms for 3-Hydroxy-2-(Thiophen-2-Yl)Chromen-4-One. RSC Adv. 2016, 6(98), 96147-96153.

205. Huang, J. D.; Yu, K.; Ma, H.; Chai, S.; Dong, B. Theoretical Investigation of ExcitedState Single and Double Proton Transfer Mechanisms for 2,5-Bis(Benzoxazol-2-Yl) Thiophene-3,4-Diol. Dye. Pigment. 2017, 141, 441-447.

206. Zhu, Q.; Wen, K.; Feng, S.; Wu, W.; An, B.; Yuan, H.; Guo, X.; Zhang, J. Theoretical Insights into the Excited-State Intramolecular Proton Transfer (ESIPT) Mechanism in a Series of Amino-Type Hydrogen-Bonding Dye Molecules Bearing the 10-Aminobenzo[h]Quinoline Chromophore. Dye. Pigment. 2017, 141, 195-201.

207. Liu, Y.-H.; Wang, S.-M.; Zhu, C.; Lin, S. H. A TDDFT Study on the Excited-State Double Proton Transfer Reaction of 8-Hydroxyquinoline Along a Hydrogen-Bonded Bridge. New J. Chem. 2017, 41(16), 8437-8442.

208. Pina, J.; Sarmento, D.; Accoto, M.; Gentili, P. L.; Vaccaro, L.; Galvão, A.; Seixas de Melo, J. S. Excited-State Proton Transfer in Indigo. J. Phys. Chem. B 2017, 121(10), 2308-2318.

209. Zhou, Q.; Du, C.; Yang, L.; Zhao, M.; Dai, Y.; Song, P. Mechanism for the ExcitedState Multiple Proton Transfer Process of Dihydroxyanthraquinone Chromophores. J. Phys. Chem. A 2017, 121(24), 4645-4651.

210. Li, C.; Ma, C.; Li, D.; Liu, Y. Excited State Intramolecular Proton Transfer (ESIPT) of 6-Amino-2-(2'-Hydroxyphenyl)Benzoxazole in Dichloromethane and Methanol: A TD-DFT Quantum Chemical Study. J. Lumin. 2016, 172, 29-33.

211. Li, C.; Yang, Y.; Ma, C.; Liu, Y. Effect of Amino Group on the Excited-State Intramolecular Proton Transfer (ESIPT) Mechanisms of 2-(2'-Hydroxyphenyl) Benzoxazole and Its Amino Derivatives. RSC Adv. 2016, 6(6), 5134-5140.

212. Zhang, X.; Liu, J. Y. Solvent Dependent Photophysical Properties and Near-Infrared Solid-State Excited State Intramolecular Proton Transfer (ESIPT) Fluorescence of 2,4,6-Trisbenzothiazolylphenol. Dye. Pigment. 2016, 125, 80-88. 
213. Zhao, J.; Chen, J.; Cui, Y.; Wang, J.; Xia, L.; Dai, Y.; Song, P.; Ma, F. A Questionable Excited-State Double-Proton Transfer Mechanism for 3-Hydroxyisoquinoline. Phys. Chem. Chem. Phys. 2015, 17(2), 1142-1150.

214. Zhao, J.; Chen, J.; Liu, J.; Hoffmann, M. R. Competitive Excited-State Single or Double Proton Transfer Mechanisms for Bis-2,5-(2-Benzoxazolyl)-Hydroquinone and Its Derivatives. Phys. Chem. Chem. Phys. 2015, 17(18), 11990-11999.

215. Zhao, J.; Yao, H.; Liu, J.; Hoffmann, M. R. New Excited-State Proton Transfer Mechanisms for 1,8-Dihydroxydibenzo[a,h]Phenazine. J. Phys. Chem. A 2015, 119(4), 681-688.

216. Liu, Y.-H.; Lan, S.-C.; Zhu, C.; Lin, S.-H. Intersystem Crossing Pathway in Quinoline-Pyrazole Isomerism: A Time-Dependent Density Functional Theory Study on Excited-State Intramolecular Proton Transfer. J. Phys. Chem. A 2015, 119(24), 6269-6274.

217. Yuan, H.; Cao, C. A Substructure-Based Topological Quantum Chemistry Approach for the Estimation of the Ultraviolet Absorption Energy of Some Substituted Linear Conjugated Compounds. Comput. Theor. Chem. 2016, 1096, 66-73.

218. Huix-Rotllant, M.; Ferre, N. Triplet State Photochemistry and the Three-State Crossing of Acetophenone within Time-Dependent Density-Functional Theory. J. Chem. Phys. 2014, 140(13), 134305.

219. Huix-Rotllant, M.; Ferre, N. Theoretical Study of the Photochemical Initiation in Nitroxide-Mediated Photopolymerization. J. Phys. Chem. A 2014, 118(25), 4464-4470.

220. Izgorodina, E. I.; Coote, M. L.; Radom, L. Trends in R-X Bond Dissociation Energies $(\mathrm{R}=\mathrm{Me}, \mathrm{Et}, \mathrm{i}-\mathrm{Pr}, \mathrm{t}-\mathrm{Bu} ; \mathrm{X}=\mathrm{H}, \mathrm{CH} 3, \mathrm{OCH} 3, \mathrm{OH}, \mathrm{F})$ : A Surprising Shortcoming of Density Functional Theory. J. Phys. Chem. A 2005, 109(33), 7558-7566.

221. Frick, E.; Schweigert, C.; Noble, B. B.; Ernst, H. A.; Lauer, A.; Liang, Y.; Voll, D.; Coote, M. L.; Unterreiner, A. N.; Barner-Kowollik, C. Toward a Quantitative Description of Radical Photoinitiator Structure-Reactivity Correlations. Macromolecules 2016, 49(1), 80-89.

222. Xiao, P.; Dumur, F.; Graff, B.; Fouassier, J. P.; Gigmes, D.; Lalevée, J. Cationic and Thiol-Ene Photopolymerization upon Red Lights Using Anthraquinone Derivatives as Photoinitiators. Macromolecules 2013, 46, 6744-6750.

223. Zhang, J.; Hill, N. S.; Lalevée, J.; Fouassier, J.; Zhao, J.; Graff, B.; Schmidt, T. W.; Kable, S. H.; Stenzel, M. H.; Coote, M. L.; et al. Multihydroxy-Anthraquinone Derivatives as Free Radical and Cationic Photoinitiators of Various Photopolymerizations under Green LED. Macromol. Rapid Commun. 2018, 39(9) 1800172.

224. Luo, J.; Liu, Y.; Yang, S.; Flourat, A. L.; Allais, F.; Han, K. Ultrafast Barrierless Photoisomerization and Strong Ultraviolet Absorption of Photoproducts in Plant Sunscreens. J. Phys. Chem. Lett. 2017, 8(5), 1025-1030.

225. Mazzone, G.; Quartarolo, A. D.; Russo, N. PDT-Correlated Photophysical Properties of Thienopyrrole BODIPY Derivatives. Theoretical Insights. Dye. Pigment. 2016, 130, 9-15.

226. Liu, B.; Lim, C. H.; Miyake, G. M. Visible-Light-Promoted C-S Cross-Coupling via Intermolecular Charge Transfer. J. Am. Chem. Soc. 2017, 139(39), 13616-13619.

227. Kozlowski, P. M.; Garabato, B. D.; Lodowski, P.; Jaworska, M. Photolytic Properties of Cobalamins: A Theoretical Perspective. Dalt. Trans. 2016, 45(11), 4457-4470.

228. Ding, B. W.; Liu, Y. J. Bioluminescence of Firefly Squid via Mechanism of Single Electron-Transfer Oxygenation and Charge-Transfer-Induced Luminescence. J. Am. Chem. Soc. 2017, 139(3), 1106-1119.

229. Tsuji, F. I. Bioluminescence Reaction Catalyzed by Membrane-Bound Luciferase in the "Firefly Squid," Watasenia Scintillans. Biochim. Biophys. Acta Biomembr. 2002, 1564(1), 189-197. 
230. Ashwood, B.; Jockusch, S.; Crespo-Hernández, C. E. Excited-State Dynamics of the Thiopurine Prodrug 6-Thioguanine: Can N9-Glycosylation Affect Its Phototoxic Activity? Molecules 2017, 22(3), 379.

231. De Simone, B. C.; Mazzone, G.; Pirillo, J.; Russo, N.; Sicilia, E. Halogen Atom Effect on the Photophysical Properties of Substituted Aza-BODIPY Derivatives. Phys. Chem. Chem. Phys. 2017, 19(3), 2530-2536.

232. McNaught, A. D.; Wilkinson, A. IUPAC. Compendium of Chemical Terminology, 2nd ed.; Blackwell Scientific Publications, 1997.

233. Golden, J. H.; Facendola, J. W.; Sylvinson, D. M. R.; Baez, C. Q.; Djurovich, P. I.; Thompson, M. E. Boron Dipyridylmethene (DIPYR) Dyes: Shedding Light on Pyridine-Based Chromophores. J. Org. Chem. 2017, 82(14), 7215-7222.

234. Lorenz, T.; Crumbach, M.; Eckert, T.; Lik, A.; Helten, H. Poly(p-Phenylene Iminoborane): A Boron-Nitrogen Analogue of Poly(p-Phenylene Vinylene). Angew. Chemie Int. Ed. 2017, 56(10), 2780-2784.

235. Margar, S. N.; Rhyman, L.; Ramasami, P.; Sekar, N. Fluorescent DifluoroboronCurcumin Analogs: An Investigation of the Electronic Structures and Photophysical Properties. Spectrochim. Acta A Mol. Biomol. Spectrosc. 2016, 152, 241-251.

236. Cabrera-González, J.; Viñas, C.; Haukka, M.; Bhattacharyya, S.; Gierschner, J.; Núñez, R. Photoluminescence in Carborane-Stilbene Triads: A Structural, Spectroscopic, and Computational Study. Chem. A Eur. J. 2016, 22(38), 13588-13598.

237. Ferrer-Ugalde, A.; Juárez-Pérez, E. J.; Teixidor, F.; Viñas, C.; Sillanpää, R.; PérezInestrosa, E.; Núñez, R. Synthesis and Characterization of New Fluorescent Styrene-Containing Carborane Derivatives: The Singular Quenching Role of a Phenyl Substituent. Chem. A Eur. J. 2012, 18(2), 544-553.

238. Weber, L.; Kahlert, J.; Brockhinke, R.; Böhling, L.; Brockhinke, A.; Stammler, H. G.; Neumann, B.; Harder, R. A.; Fox, M. A. Luminescence Properties of C-DiazaborolylOrtho-Carboranes as Donor-Acceptor Systems. Chem. A Eur. J. 2012, 18(27), 8347-8357.

239. Momeni, M. R.; Brown, A. Why Do TD-DFT Excitation Energies of BODIPY/ Aza-BODIPY Families Largely Deviate From Experiment? Answers From Electron Correlated and Multireference Methods. J. Chem. Theory Comput. 2015, 11(6), 2619-2632.

240. Fujikawa, T.; Segawa, Y.; Itami, K. Synthesis, Structures, and Properties of $\pi$-Extended Double Helicene: A Combination of Planar and Nonplanar $\pi$-Systems. J. Am. Chem. Soc. 2015, 137(24), 7763-7768.

241. Maier, T. M.; Bahmann, H.; Kaupp, M. Efficient Semi-Numerical Implementation of Global and Local Hybrid Functionals for Time-Dependent Density Functional Theory. J. Chem. Theory Comput. 2015, 11(9), 4226-4237.

242. Maier, T. M.; Bahmann, H.; Arbuznikov, A. V.; Kaupp, M. Validation of Local Hybrid Functionals for TDDFT Calculations of Electronic Excitation Energies. J. Chem. Phys. 2016, 144(7), 0-14.

243. Schreiber, M.; Silva-Junior, M. R.; Sauer, S. P. A.; Thiel, W. Benchmarks for Electronically Excited States: CASPT2, CC2, CCSD, and CC3. J. Chem. Phys. 2008, $128(13), 134110$.

244. Silva-Junior, M. R.; Schreiber, M.; Sauer, S. P. A.; Thiel, W. Benchmarks for Electronically Excited States: Time-Dependent Density Functional Theory and Density Functional Theory Based Multireference Configuration Interaction. J. Chem. Phys. 2008, 129(10), 104103.

245. Zhekova, H.; Krykunov, M.; Autschbach, J.; Ziegler, T. Applications of Time Dependent and Time Independent Density Functional Theory to the First $\pi$ to $\Pi^{*}$ Transition in Cyanine Dyes. J. Chem. Theory Comput. 2014, 10(8), 3299-3307. 
246. Cao, J.; London, G.; Dumele, O.; Von Wantoch Rekowski, M.; Trapp, N.; Ruhlmann, L.; Boudon, C.; Stanger, A.; Diederich, F. The Impact of Antiaromatic Subunits in $[4 \mathrm{n}+2] \pi$-Systems: Bispentalenes with $[4 \mathrm{n}+2] \pi$-Electron Perimeters and Antiaromatic Character. J. Am. Chem. Soc. 2015, 137(22), 7178-7188.

247. Etienne, T.; Assfeld, X.; Monari, A. Toward a Quantitative Assessment of Electronic Transitions' Charge-Transfer Character. J. Chem. Theory Comput. 2014, 10(9), 3896-3905.

248. Ji, L.; Edkins, R. M.; Sewell, L. J.; Beeby, A.; Batsanov, A. S.; Fucke, K.; Drafz, M.; Howard, J. A. K.; Moutounet, O.; Ibersiene, F.; et al. Experimental and Theoretical Studies of Quadrupolar Oligothiophene-Cored Chromophores Containing Dimesitylboryl Moieties as $\pi$-Accepting End-Groups: Syntheses, Structures, Fluorescence, and One- and Two-Photon Absorption. Chem. A Eur. J. 2014, 20(42), 13618-13635.

249. Arulmozhiraja, S.; Coote, M. L.; Lu, D.; Salem, G.; Wild, S. B. Origin of the Unusual Ultraviolet Absorption of Arsenicin A. J. Phys. Chem. A 2011, 115(17), 4530-4534.

250. Samanta, P. K.; Kim, D.; Coropceanu, V.; Brédas, J. L. Up-Conversion Intersystem Crossing Rates in Organic Emitters for Thermally Activated Delayed Fluorescence: Impact of the Nature of Singlet vs Triplet Excited States. J. Am. Chem. Soc. 2017, 139(11), 4042-4051.

251. Cai, X.; Li, X.; Xie, G.; He, Z.; Gao, K.; Liu, K.; Chen, D.; Cao, Y.; Su, S.-J. "RateLimited Effect" of Reverse Intersystem Crossing Process: The Key for Tuning Thermally Activated Delayed Fluorescence Lifetime and Efficiency Roll-off of Organic Light Emitting Diodes. Chem. Sci. 2016, 7(7), 4264-4275.

252. Gómez-Bombarelli, R.; Aguilera-Iparraguirre, J.; Hirzel, T. D.; Duvenaud, D.; Maclaurin, D.; Blood-Forsythe, M. A.; Chae, H. S.; Einzinger, M.; Ha, D. G.; Wu, T.; et al. Design of Efficient Molecular Organic Light-Emitting Diodes by a High-Throughput Virtual Screening and Experimental Approach. Nat. Mater. 2016, 15(10), 1120-1127.

253. Valchanov, G.; Ivanova, A.; Tadjer, A.; Chercka, D.; Baumgarten, M. Understanding the Fluorescence of TADF Light-Emitting Dyes. J. Phys. Chem. A 2016, 120(35), 6944-6955.

254. Chen, X. K.; Zhang, S. F.; Fan, J. X.; Ren, A. M. Nature of Highly Efficient Thermally Activated Delayed Fluorescence in Organic Light-Emitting Diode Emitters: Nonadiabatic Effect Between Excited States. J. Phys. Chem. C 2015, 119(18), 9728-9733.

255. Zhang, Q.; Kuwabara, H.; Potscavage, W. J.; Huang, S.; Hatae, Y.; Shibata, T.; Adachi, C. Anthraquinone-Based Intramolecular Charge-Transfer Compounds: Computational Molecular Design, Thermally Activated Delayed Fluorescence, and Highly Efficient Red Electroluminescence. J. Am. Chem. Soc. 2014, 136(52), 18070-18081.

256. Walter, C.; Krämer, V.; Engels, B. On the Applicability of Time-Dependent Density Functional Theory (TDDFT) and Semiempirical Methods to the Computation of Excited-State Potential Energy Surfaces of Perylene-Based Dye-Aggregates. Int. J. Quantum Chem. 2017, 117(6), 1-14.

257. Weber, W.; Thiel, W. Orthogonalization Corrections for Semiempirical Methods. Theor. Chem. Acc. 2000, 103(6), 495-506.

258. Huang, S.; Zhang, Q.; Shiota, Y.; Nakagawa, T.; Kuwabara, K.; Yoshizawa, K.; Adachi, C. Computational Prediction for Singlet- and Triplet-Transition Energies of Charge-Transfer Compounds. J. Chem. Theory Comput. 2013, 9(9), 3872-3877.

259. Qian, H.; Cousins, M. E.; Horak, E. H.; Wakefield, A.; Liptak, M. D.; Aprahamian, I. Suppression of Kasha's Rule as a Mechanism for Fluorescent Molecular Rotors and Aggregation-Induced Emission. Nat. Chem. 2017, 9(1), 83-87. 
260. Escudero, D. Revising Intramolecular Photoinduced Electron Transfer (PET) From First-Principles. Acc. Chem. Res. 2016, 49(9), 1816-1824.

261. Shepard, S. G.; Fatur, S. M.; Rappé, A. K.; Damrauer, N. H. Highly Strained Iron(II) Polypyridines: Exploiting the Quintet Manifold to Extend the Lifetime of MLCT Excited States. J. Am. Chem. Soc. 2016, 138(9), 2949-2952.

262. Nobusue, S.; Miyoshi, H.; Shimizu, A.; Hisaki, I.; Fukuda, K.; Nakano, M.; Tobe, Y. Tetracyclopenta[Def,Jkl,Pqr,Vwx]Tetraphenylene: A Potential Tetraradicaloid Hydrocarbon. Angew. Chem. Int. Ed. 2015, 54(7), 2090-2094.

263. Hu, P.; Lee, S.; Herng, T. S.; Aratani, N.; Gonçalves, T. P.; Qi, Q.; Shi, X.; Yamada, H.; Huang, K. W.; Ding, J.; et al. Toward Tetraradicaloid: The Effect of Fusion Mode on Radical Character and Chemical Reactivity. J. Am. Chem. Soc. 2016, 138(3), 1065-1077.

264. Lou, Z.; Li, P.; Han, K. Redox-Responsive Fluorescent Probes With Different Design Strategies. Acc. Chem. Res. 2015, 48(5), 1358-1368.

265. Daday, C.; Curutchet, C.; Sinicropi, A.; Mennucci, B.; Filippi, C. ChromophoreProtein Coupling beyond Nonpolarizable Models: Understanding Absorption in Green Fluorescent Protein. J. Chem. Theory Comput. 2015, 11(10), 4825-4839.

266. Wu, Q.; Zhang, T.; Peng, Q.; Wang, D.; Shuai, Z. Aggregation Induced Blue-Shifted Emission-The Molecular Picture From a QM/MM Study. Phys. Chem. Chem. Phys. 2014, 16(12), 5545-5552.

267. Johnson, L. E.; Dalton, L. R.; Robinson, B. H. Optimizing Calculations of Electronic Excitations and Relative Hyperpolarizabilities of Electrooptic Chromophores. Acc. Chem. Res. 2014, 47(11), 3258-3265.

268. Pescitelli, G.; Bruhn, T. Good Computational Practice in the Assignment of Absolute Configurations by TDDFT Calculations of ECD Spectra. Chirality 2016, $28,466-474$.

269. Longhi, G.; Castiglioni, E.; Koshoubu, J.; Mazzeo, G.; Abbate, S. Circularly Polarized Luminescence: A Review of Experimental and Theoretical Aspects. Chirality 2016, 28, 696-707.

270. Avila Ferrer, F. J.; Cerezo, J.; Soto, J.; Improta, R.; Santoro, F. First-Principle Computation of Absorption and Fluorescence Spectra in Solution Accounting for Vibronic Structure, Temperature Effects and Solvent Inhomogenous Broadening. Comput. Theor. Chem. 2014, 1040-1041, 328-337.

271. Rozzi, C. A.; Falke, S. M.; Spallanzani, N.; Rubio, A.; Molinari, E.; Brida, D.; Maiuri, M.; Cerullo, G.; Schramm, H.; Christoffers, J.; et al. Quantum Coherence Controls the Charge Separation in a Prototypical Artificial Light Harvesting System. Nat. Commun. 2013, 4, 1602-1607.

272. Perdew, J. P.; Zunger, A. Self-Interaction Correction to Density-Functional Approximations for Many-Electron Systems. Phys. Rev. B 1981, 23(10), 5048-5079.

273. Santhanamoorthi, N.; Lo, C. M.; Jiang, J. C. Molecular Design of Porphyrins for Dye-Sensitized Solar Cells: A DFT/TDDFT Study. J. Phys. Chem. Lett. 2013, 4(3), 524-530.

274. Karthikeyan, S.; Lee, J. Y. Zinc-Porphyrin Based Dyes for Dye-Sensitized Solar Cells. J. Phys. Chem. A 2013, 117(42), 10973-10979.

275. Guido, C. A.; Knecht, S.; Kongsted, J.; Mennucci, B. Benchmarking Time-Dependent Density Functional Theory for Excited State Geometries of Organic Molecules in Gas-Phase and in Solution. J. Chem. Theory Comput. 2013, 9(5), 2209-2220.

276. Sousa, C.; De Graaf, C.; Rudavskyi, A.; Broer, R.; Tatchen, J.; Etinski, M.; Marian, C. M. Ultrafast Deactivation Mechanism of the Excited Singlet in the Light-Induced Spin Crossover of $[\mathrm{Fe}(2,2-B i p y r i d i n e) 3] 2+$. Chem. A Eur. J. 2013, 19(51), 17541-17551. 
277. Cerezo, J.; Zúñiga, J.; Requena, A.; Ávila Ferrer, F. J.; Santoro, F. Harmonic Models in Cartesian and Internal Coordinates to Simulate the Absorption Spectra of Carotenoids at Finite Temperatures. J. Chem. Theory Comput. 2013, 9(11), 4947-4958.

278. Long, L.; Zhou, L.; Wang, L.; Meng, S.; Gong, A.; Du, F.; Zhang, C. A CoumarinBased Fluorescent Probe for Biological Thiols and Its Application for Living Cell Imaging. Org. Biomol. Chem. 2013, 11(47), 8214.

279. Baiardi, A.; Bloino, J.; Barone, V. General Time Dependent Approach to Vibronic Spectroscopy Including Franck-Condon, Herzberg-Teller, and Duschinsky Effect. J. Chem. Theory Comput. 2013, 9(9), 4097-4115.

280. Small, G. J. Herzberg-Teller Vibronic Coupling and the Duschinsky Effect. J. Chem. Phys. 1971, 54(8), 3300-3306.

281. De Mitri, N.; Monti, S.; Prampolini, G.; Barone, V. Absorption and Emission Spectra of a Flexible Dye in Solution : A Computational Time-Dependent Approach. J. Chem. Theory Comput. 2013, 9, 4507-4516.

282. Li, Z.; Suo, B.; Zhang, Y.; Xiao, Y.; Liu, W. Combining Spin-Adapted Open-Shell TD-DFT With Spin-Orbit Coupling. Mol. Phys. 2013, 111(24), 3741-3755.

283. Li, Z.; Liu, W. Critical Assessment of TD-DFT for Excited States of Open-Shell Systems: I. Doublet-Doublet Transitions. J. Chem. Theory Comput. 2016, 12(1), $238-260$.

284. Li, Z.; Liu, W. Critical Assessment of Time-Dependent Density Functional Theory for Excited States of Open-Shell Systems: II. Doublet-Quartet Transitions. J. Chem. Theory Comput. 2016, 12, 2517-2527.

285. Suo, B.; Shen, K.; Li, Z.; Liu, W. Performance of TD-DFT for Excited States of Open-Shell Transition Metal Compounds. J. Phys. Chem. A 2017, 121, 3929-3942.

286. Fan, D.; Yi, Y.; Li, Z.; Liu, W.; Peng, Q.; Shuai, Z. Solvent Effects on the Optical Spectra and Excited-State Decay of Triphenylamine-Thiadiazole With Hybridized Local Excitation and Intramolecular Charge Transfer. J. Phys. Chem. A 2015, 119, 5233-5240.

287. Zhang, C.; Yuan, D.; Guo, Y.; Li, S. Efficient Implementation of Local Excitation Approximation for Treating Excited States of Molecules in Condensed Phase. J. Chem. Theory Comput. 2014, 10(12), 5308-5317.

288. Milanese, J. M.; Provorse, M. R.; Alameda, E.; Isborn, C. M. Convergence of Computed Aqueous Absorption Spectra With Explicit Quantum Mechanical Solvent. J. Chem. Theory Comput. 2017, 13(5), 2159-2171.

289. Martínez-Fernández, L.; Pepino, A. J.; Segarra-Martí, J.; Jovaišaitei, J.; Vaya, I.; Nenov, A.; Markovitsi, D.; Gustavsson, T.; Banyasz, A.; Garavelli, M.; et al. Photophysics of Deoxycytidine and 5-Methyldeoxycytidine in Solution: A Comprehensive Picture by Quantum Mechanical Calculations and Femtosecond Fluorescence Spectroscopy. J. Am. Chem. Soc. 2017, 139(23), 7780-7791.

290. Szabla, R.; Kruse, H.; Šponer, J.; Góra, R. W. Water-Chromophore Electron Transfer Determines the Photochemistry of Cytosine and Cytidine. Phys. Chem. Chem. Phys. 2017, 19(27), 17531-17537.

291. Li, Q.; Mennucci, B.; Robb, M. A.; Blancafort, L.; Curutchet, C. Polarizable QM/MM Multiconfiguration Self-Consistent Field Approach With State-Specific Corrections: Environment Effects on Cytosine Absorption Spectrum. J. Chem. Theory Comput. 2015, 11(4), 1674-1682.

292. Spata, V. A.; Matsika, S. Bonded Excimer Formation in $\pi$-Stacked 9-Methyladenine Dimers. J. Phys. Chem. A 2013, 117(36), 8718-8728.

293. Fang, H.; Kim, Y. Excited-State Tautomerization of 7-Azaindole in Nonpolar Solution: A Theoretical Study Based on Liquid-Phase Potential Surfaces of Mean Force. J. Chem. Theory Comput. 2013, 9(8), 3557-3566. 
294. Zuehlsdorff, T. J.; Haynes, P. D.; Payne, M. C.; Hine, N. D. M. Predicting Solvatochromic Shifts and Colours of a Solvated Organic Dye: The Example of Nile Red. J. Chem. Phys. 2017, 146(12), 124504.

295. Zuehlsdorff, T. J.; Haynes, P. D.; Hanke, F.; Payne, M. C.; Hine, N. D. M. Solvent Effects on Electronic Excitations of an Organic Chromophore. J. Chem. Theory Comput. 2016, 12(4), 1853-1861.

296. Chang, X. P.; Li, C. X.; Xie, B. B.; Cui, G. Photoprotection Mechanism of P-Methoxy Methylcinnamate: A CASPT2 Study. J. Phys. Chem. A 2015, 119(47), 11488-11497.

297. Charlton, R. J.; Fogarty, R.; Bogatko, S.; Horsfield, A. P.; Haynes, P. D.; Hine, N. D. M. Implicit and Explicit p-Terphenyl Host Effects on Excitons in Pentacene Derivatives. J. Chem. Phys. 2018, 148, 104108.

298. Marazzi, M.; Gattuso, H.; Monari, A. Nile Blue and Nile Red Optical Properties Predicted by TD-DFT and CASPT2 Methods: Static and Dynamic Solvent Effects. Theor. Chem. Acc. 2016, 135(3), 1-11.

299. Mewes, J. M.; You, Z. Q.; Wormit, M.; Kriesche, T.; Herbert, J. M.; Dreuw, A. Experimental Benchmark Data and Systematic Evaluation of Two a Posteriori, Polarizable-Continuum Corrections for Vertical Excitation Energies in Solution. J. Phys. Chem. A 2015, 119(21), 5446-5464.

300. Isborn, C. M.; Mar, B. D.; Curchod, B. F. E.; Tavernelli, I.; Martínez, T. J. The Charge Transfer Problem in Density Functional Theory Calculations of Aqueously Solvated Molecules. J. Phys. Chem. B 2013, 117(40), 12189-12201.

301. Tomasi, J.; Mennucci, B.; Cammi, R. Quantum Mechanical Continuum Solvation Models. Chem. Rev. 2005, 105(8), 2999-3093.

302. Caricato, M. A Comparison Between State-Specific and Linear-Response Formalisms for the Calculation of Vertical Electronic Transition Energy in Solution With the CCSD-PCM Method. J. Chem. Phys. 2013, 139(4), 044116.

303. Li, X. Y. An Overview of Continuum Models for Nonequilibrium Solvation: Popular Theories and New Challenge. Int. J. Quantum Chem. 2015, 115(11), 700-721.

304. Fukuda, R.; Ehara, M. Electronic Excitation of Molecules in Solution Calculated Using the Symmetry-Adapted Cluster-Configuration Interaction Method in the Polarizable Continuum Model. AIP Conf. Proc. 2015, 1702.

305. Caricato, M. A Corrected-Linear Response Formalism for the Calculation of Electronic Excitation Energies of Solvated Molecules With the CCSD-PCM Method. Comput. Theor. Chem. 2014, 1040-1041, 99-105.

306. Mewes, J. M.; Herbert, J. M.; Dreuw, A. On the Accuracy of the General, StateSpecific Polarizable-Continuum Model for the Description of Correlated Groundand Excited States in Solution. Phys. Chem. Chem. Phys. 2017, 19(2), 1644-1654.

307. Hill, N. S.; Coote, M. L. Internal Oriented Electric Fields as a Strategy for Selectively Modifying Photochemical Reactivity. J. Am. Chem. Soc. 2018, 140, 17800-17804.

\section{Further reading}

308. Amatatsu, Y. Theoretical Design of a Light-Driven Molecular Rotary Motor with Low Energy Helical Inversion: 9-(5-Methyl-2-Phenyl-2-Cyclopenten-1-Ylidene)-9HFluorene. J. Phys. Chem. A 2011, 115(46), 13611-13618.

309. Amatatsu, Y. Theoretical Study of Topographical Features Around the Conical Intersections of Fluorene-Based Light-Driven Molecular Rotary Motor. J. Phys. Chem. A 2013, 117(17), 3689-3696.

310. Amatatsu, Y. Theoretical Study of Topographical Features around the Conical Intersections of 9-(2-Cyclopenten-1-Ylidene)-9H-Fluorene. J. Phys. Chem. A 2013, 117(47), 12529-12539. 
311. Amatatsu, Y. Theoretical Study on Conformational-Dependent Photochemical Behaviors of a Fluorene-Based Light-Driven Molecular Rotary Motor. Bull. Chem. Soc. Jpn. 2015, 88(10), 1417-1430.

312. Amatatsu, Y. Theoretical Study on the Torsional Direction of Simple Ethylenoids after Electronic Relaxation at the Conical Intersection in the Cis-Trans Photoisomerization. Chem. Phys. Lett. 2015, 631-632, 47-53.

313. Adamska, L.; Nayyar, I.; Chen, H.; Swan, A. K.; Oldani, N.; Fernandez-Alberti, S.; Golder, M. R.; Jasti, R.; Doorn, S. K.; Tretiak, S. Self-Trapping of Excitons, Violation of Condon Approximation, and Efficient Fluorescence in Conjugated Cycloparaphenylenes. Nano Lett. 2014, 14(11), 6539-6546. 